UCRL-16613

FALL 1965

\title{
SEMIANNUAL REPORT BIOLOGY and MEDICINE
}

\section{TWO-WEEK LOAN COPY}

This is a Library Circulating Copy which may be borrowed for two weeks. For a personal retention copy, call Tech. Info. Division, Ext. 5545

\section{DONNER LABORATORY and DONNER PAVILION}




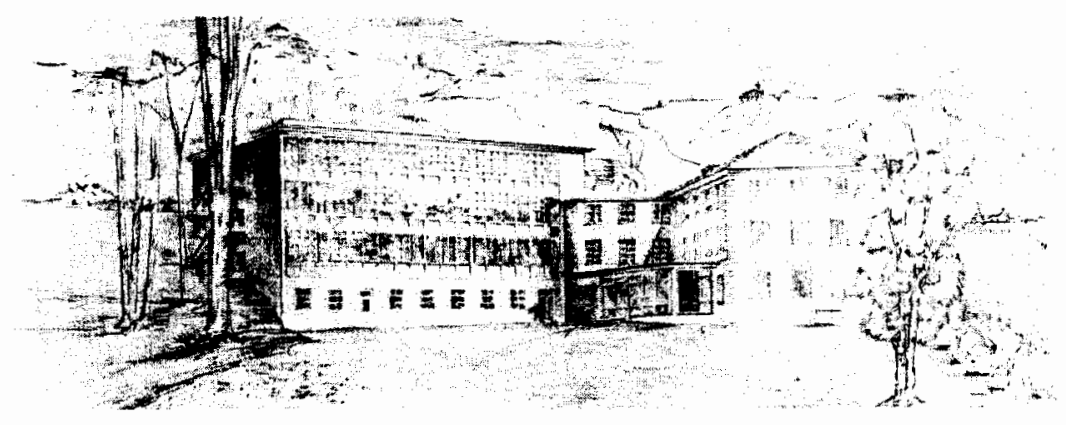

\title{
DONNER LABORATORY AND DONNER PAVILION
}

\author{
LAWRENCE RADIATION LABORATORY - UNIVERSITY OF CALIFORNIA
}

BERKELEY, CALIFORNIA

\section{SEMIANNUAL REPORT - BIOLOGY AND MEDICINE}

FALL 1965

$$
\text { (January 7, 1966) }
$$

John H. Lawrence, M.D., Editor

Tove Neville, Associate Editor 


\title{
Radiation Doses on Manned Space Missions
}

\author{
Stanley B. Curtis
}

Two of the important factors determining the radiation doses our astronauts will encounter on space missions to be undertaken in the next few years are orbit or trajectory location and spacecraft shielding thickness. In general, earth-orbiters with orbital inclinations up to $30^{\circ}$ and altitudes in the 100 to 500 nautical mile range will be free from dangerous levels of radiation. This situation changes as the altitude or orbital inclination increases. On the mission to the moon, the Apollo mission, the danger will come almost entirely from the large solar flares that eject high fluxes of protons and alpha particles capable of penetrating the walls of a lightly shielded vehicle. This paper will review several consequences of varying the spacecraft trajectory and indicate what dose rates are expected on missions planned in the next few years.

\section{EARTH-ORBITERS}

The Gemini and proposed ORL (Orbital Research Laboratory) spacecraft will have similar amounts of shielding and similar orbital characteristics. There will be 1 to $2 \mathrm{~g} / \mathrm{cm}^{2}$ of shielding provided by the spacecraft walls and equipment. The altitudes will be in the 100 to 300 nautical mile range, and the orbital inclination will probably be close to $30^{\circ}$. If any of these parameters are changed significantly, a reassessment of the radiation doses will have to be made. The Air Force MOL (Manned Orbital Laboratory) program will include polar flights. This increase in orbital inclination to $90^{\circ}$ decreases the effective magnetic shielding of the geomagnetic field and allows fluxes from solar-particle events to reach the spacecraft and to increase the dose rate significantly.

The largest contribution to the dose in low inclination earth-orbiters (around $30^{\circ}$ ) will come from the protons and electrons encountered in the South Atlantic anomaly. This is a region over the South Atlantic Ocean where an anomaly in the geomagnetic field causes the particles in the trapped radiation belts to dip closer to the earth. A spacecraft will typically traverse the anomaly six times every 16 orbits. The average duration of exposure is about 10 to $15 \mathrm{~min}$. In the past few years, electrons have been predominant in this anomaly because of the artificial radiation belt resulting from the high-altitude nuclear explosions in 1962 . By the time of this writing, the electrons have decayed to a point where the predominant contribution to the radiation dose behind 1 to $2 \mathrm{~g} / \mathrm{cm}^{2}$ of shielding is from the proton component of the natural radiation belt. It is interesting to note that there is little dependence of this component to the daily dose on altitude in the range 160 to 500 nautical miles and on orbital inclination from $30^{\circ}$ to $90^{\circ}$ (1). This is because the only source of belt radiation in this range is the South Atlantic anomaly. The small range of altitudes accounts for the lack of dependence 
on altitude. The lack of dependence on inclination is because it matters little whether the spacecraft traverses the anomaly from north to south $\left(90^{\circ}\right.$ inclination) or from northwest to southeast $\left(45^{\circ}\right.$ inclination).

The added source of radiation at high orbital inclination $\left(>60^{\circ}\right)$ comes not from the radiation belt but from the solar particles that are able to penetrate deeply into the polar regions during a solar flare event and, to a lesser extent, from the galactic cosmic rays. At high orbital inclinations, the geomagnetic field ceases to be an effective shield against lowenergy solar particles and the low-energy particles in the galactic cosmic rays. In fact, at 200 nautical miles, the dose accumulated in three days during a large solar-particle event might be more than three orders of magnitude greater in a polar orbit than in a $30^{\circ}$ inclination orbit. The galactic dose will be two to three times greater in a polar orbit than at $30^{\circ}$. This variance with orbital inclination is due entirely to the variance of magnetic shielding supplied by the earth's magnetic field.

\section{TYPICAL DOSE RATES IN THE EARTH-ORBITERS}

Comprehensive computer codes (2) are available for estimating the dose rates within spacecraft orbiting the earth. These codes are probably as accurate as the available particle flux and spectral data that are used in the dose calculation. Experimental data are also available from the Gemini IV flight and will undoubtedly be available soon from the longer Gemini $\mathrm{V}$ and VII flights as well. (Total doses of between 100 and 200 millirads for the 14 day flight of Gemini VII were reported in a preliminary report at the 1965 AAAS meeting by the astronauts' physician, Dr. Charles Berry.) The Gemini IV results (3) indicate an average dose rate of 15 to 16 millirads/day from the South Atlantic anomaly (95\% of this from protons) and 3.5 millirads/day from the galactic cosmic rays for a total of about 20 millirads/day. The instantaneous dose rate rose to $100 \mathrm{millirads} / \mathrm{hr}$ in the heart of the anomaly. These results are consistent with the computer code results and indicate there is very little radiation exposure in the Gemini orbits.

As has been indicated above, the magnetic shielding provided by the earth's magnetic field for low-inclination orbits effectively shields out the low-energy solar particles during a solar-flare event. The increased dose rate from a large solar-particle event might add between 1 and 10 millirads/day average for some three days, or as much as the galactic cosmic rays contribute in the same period of time. Thus, it can be seen that the radiation doses are negligible for low inclinations (i.e., less than 60\%) and low altitude (100 to 500 nautical miles). On the other hand, for a polar orbit, the dose rate from a large solar-particle event might reach an average rate of $15 \mathrm{rads} /$ day for three days or $45 \mathrm{rads}$ total skin dose behind $2 \mathrm{~g} / \mathrm{cm}^{2}$ aluminum shielding. It should be pointed out that this radiation will be attenuated rapidly as it penetrates the body, thus producing perhaps $10 \%$ of this dose at the body mid-line (1).

\section{THE APOLLO MISSION}

The greatest radiation hazard on the Apollo mission will come if the astronauts are caught in the lunar excursion module (LEM) or on the lunar surface in a large solar-particle event. The command module, or "mother ship", which remains with one astronaut on board 
orbiting the moon during the lunar landing, averages about 4 to $6 \mathrm{~g} / \mathrm{cm}^{2}$ of shielding and so provides sufficient shielding from the solar radiation, as will be seen below. A large solid angle of the LEM, on the other hand, is shielded with only $0.16 \mathrm{~g} / \mathrm{cm}^{2}$ shielding. This plus the spacesuit shielding of another $0.16 \mathrm{~g} / \mathrm{cm}^{2}$ gives the astronauts only $0.32 \mathrm{~g} / \mathrm{cm}^{2}$ shielding over almost $2 \pi$ steradians. Traversal of the trapped radiation belts in the command module will contribute less than 2 rads (4), and the galactic cosmic ray dose will not exceed 40 millirads per day even during periods of peak dose from that source. The maximum permissible emergency doses published for the Apollo mission are as follows (5):

Skin of the whole body - $700 \mathrm{rem}$

Blood forming organs (BFO) and eyes - 200 rem

Feet, ankles and hands - 980 rem

It has been estimated by the radiation group at the Manned Spacecraft Center at Houston that the probability of receiving a dose as large as this is 1 in 1,000 in the command module and 1 in 100 on the lunar surface (i. e., in the LEM) if solar flare warning is disregarded (6).

Recent work by the Manned Spacecraft Center at Houston (7) has considered the following points:

1) The doses expected when large solar-particle events occur.

2) The predictability of such events.

3) The course of action needed, if any, during such events. The first point was examined by calculating the doses that might have been received by the astronauts had the Apollo mission taken place during one of the large events of the last several years. It is well known that most of the particles in the steep energy spectrum are absorbed in the walls of the spacecraft, so that the exact shielding configuration available plus the selfshielding of the astronauts themselves are very important factors in determining the resulting radiation dose at a point within the body $(4,8)$. Shielding studies of the command module and LEM have been made, using an adequate sectoring of the command module but a rather crude sectoring of the LEM. Work is continuing at the present time to provide a better sectoring job of the LEM. The man model chosen to provide the body self-shielding was also crude and is being replaced by a more sophisticated representation. Combining the preliminary results (7) with the data on several of the recent large solar-particle events (9) we estimate total doses (i.e., from protons and alpha particles) in the command module of 20 to 50 rem at the skin of the chest, 15 to $30 \mathrm{rem}$ at the eye, and 3 to $9 \mathrm{rem}$ at the BFO. In the LEM, we find 350 to $800 \mathrm{rem}$ at the skin of the chest, 100 to $180 \mathrm{rem}$ at the eye and 3 to $12 \mathrm{rem}$ at the BFO. Several of these large events followed closely on one another within a week or ten days, so we see that it is possible in the LEM for moderate erythema to occur if the astronauts are caught by a series of solar events. The eye may also receive unacceptable levels of radiation, although cataract formation is a delayed effect and probably would not jeopardize the success of the mission. It should be mentioned in connection with these calculations that the following RBE vs. LET (linear energy transfer) relationship was assumed (10):

$$
\operatorname{RBE}(\epsilon)=0.9+0.05 \epsilon \text { where } \epsilon \text { is } \mathrm{LET} \text { in } \mathrm{keV} / \mu \text {. }
$$

Also, secondary particles produced by nuclear interactions within the shielding were not taken into account. 
From the above considerations, it has been decided that some action by the astronauts should be taken if it becomes probable that such high doses will be received while they are in the LEM. This has led to consideration of solar-event prediction reliability. It is well known that a certain type of radio emission (synchrotron radiation) is emitted when electrons are accelerated within the magnetic fields near the solar surface. These radio waves can be detected on earth. A network of three warning stations is being set up, each looking at three radio frequencies. There will be one station on the northwest coast of Australia, one at the NASA Manned Spacecraft Center in Houston and one in the Canary Islands. With these stations, 24-hr coverage may be maintained. It is felt that the time-integrated energy received from the sun at the selected frequencies $(1420,2780$, and $5000 \mathrm{Mc} / \mathrm{sec}$ ) will provide a reliable indication of the numbers of electrons and perhaps protons being accelerated. Such real time measurements, plus optical observations and previous knowledge of the plage area and activity of the sunspot group producing the flare, will give an early indication (within one hour of flare commencement) of the magnitude of the solar-particle event. If the estimation is that the event will produce dangerous radiation levels (which have yet to be agreed upon) in the vicinity of the earth-moon system, it will be recommended that the astronauts leave the lunar surface as soon as possible and proceed to a rendezvous with the command module. The time to accomplish this will vary from $21 / 2$ to $4 \mathrm{hr}$, depending on where the command module is in relation to the LEM at the time the decision is made. The problem of a "false alarm", $i . e .$, an unnecessary early return to the command module, has not been solved.

\section{SUMMARY}

In conclusion, we see that the Gemini and ORL missions will receive on the order of 20 millirads/day in low altitude (100 to 500 nautical miles) and low inclination $\left(30^{\circ}\right)$ orbits, most of this coming from the protons in the South Atlantic anomaly. There is no danger from solar-particle events because of the strong magnetic shielding available from the geomagnetic field. For the polar orbit of MOL, however, considerably larger doses are expected during solar-particle events, reaching perhaps a 50-rad skin dose in a three-day period with $10 \%$ of this at the body mid-Iine. On the Apollo mission, the greatest danger comes if the astronauts are caught in a solar-particle event on the lunar surface or in the relatively lightly shielded LEM. It has been decided to allow for this possibility by setting up a solar particle warning system and providing the mission with the option of a quick return to the command module if it is predicted that the dose rate will reach unacceptable levels.

\section{REFERENCES}

1. Langham, W. H., et a1.; Aerospace Medicine 36: No. 2, Section II, 1965.

2. Barton, J. A., and Mar, B. W. ; Computer Codes for Space Radiation Environment and Shielding, Vol. 1, Aerospace Division, Boeing Company Report, No. WLTDR-64-71, 1964.

3. Schneider, M. F.; Janni, J. F., and Radke, G. E.; Manned Space Flight Experiments Symposium, Gemini Missions III and IV, NASA Manned Spacecraft Center, p. 171, 1965.

4. Dye, D. L., and Wilkinson, M. ; Science 147:19, 1965.

5. Billingham, J.; Second Symposium on Protection Against Radiations in Space, NASA SP-71; 139, 1965. 
6. Modisette, J. L.; Second Symposium on Protection Against Radiations in Space, NASA SP-71; 147, 1965.

7. Robbins, D.; private communication, 1965.

8. Curtis, S. B.; The Physical Characteristics of Solar Flares, Workshop Conference on Space Radiation Biology, September 7-10, 1965, to be published in Radiation Res.

9. Webber, W. R.; An Evaluation of the Radiation Hazard Due to Solar-Particle Events, D2-90469, The Boeing Company, 1964.

10. Report of the RBE Committee to the International Commissions on Radiological Protection and on Radiological Units and Measurements; Health Phys. 9:357, 1963.

Received January, 1966. 


\title{
Heavy-Particle Studies with Silicon Detectors
}

\author{
Mudundi R. Raju
}

The response of lithium-drifted silicon detectors was studied with a view to determining applications of these detectors to radiobiologic problems. Until recently these detectors had only limited applications because they were available only with limited thickness. Now the technology has been improved, and detectors several millimeters thick are available (see reference 1, for example). The availability of these detectors has revolutionized radia tion detection and spectroscopy (2). The use of a solid as a detector is very attractive because the sensitive layer can be quite thin and yet possess a high stopping power. Another advantage is the low energy used to produce one hole-electron pair ( $3.6 \mathrm{eV}$ in silicon): nearly ten times as much charge is produced for a given energy loss in silicon as in gas, which leads to small statistical fluctuations in the number of pairs and to improved energy resolution over gas filled counters. The intrinsically high speed of the device is due to the high mobility of the carriers in the electric field, coupled with the short distance between electrodes. An attractive feature of these detectors is that they operate as true energy devices and do not exhibit saturation effects as do scintillation counters.

Depending on the energy of the particles and the thickness of the detector, a given detector may be used to measure either their energy-loss distribution or energy distribution. If the detector is thin, so that the energy deposited by the particle in it is very small compared with the energy of the particles, the detector can be used to measure the energy-loss distribution. On the other hand, if the detector is thick enough to stop all the particles in the beam, then it can be used to measure energy distribution of the particles. The use of these detectors for the study of pion beams and to measure the pion-star energy distribution in silicon has been reported (3). The application of these detectors for experimental verification of the Landau theory of energy-loss distribution has also been reported (4).

In this investigation the response of these detectors to high-energy alpha and proton beams, and the intermediate energies, obtained by placing absorbing materials in the path of the primary beam, have been studied.

The Bragg peak of heavy particles is often used for therapy and for radiobiological investigations. Besides knowing the dose at the Bragg-peak position, one needs also to know the linear energy transfer (LET) distribution at this position. In order to evaluate the LET distribution of heavy particles at the Bragg-peak position, the energy distribution of these particles was measured. 


\section{CA LIBRATION}

The experimental setup is shown in Fig. 1. The test-pulse generator was used to simulate detector pulses and to check the linearity of the electronic system. An ${ }^{241}$ Am alpha source and a ${ }^{207} \mathrm{Bi}$ internal conversion electron source were used for calibrating the pulsegenerator output in terms of energy. Calibration and linearity checks were made for every experiment.

\section{RESULTS}

BEAM: HELIUM NUCLEI The Berkeley heavy-ion linear accelerator (Hilac) accelerates nuclei to an energy of $10.40 \pm 0.2 \mathrm{MeV}$ per nucleon. For helium nuclei the energy of the primary beam is $41.6 \mathrm{MeV}$. The beam is extracted through a vacuum column with a $1-\mathrm{mil}$ aluminum window. The detector was enclosed by a housing (in order that a vacuum can be maintained) with a 1-mil Mylar window. The entire detector holder assembly was surrounded with a 1/4-mil aluminum electronic shield. By applying the correction for the degradation of the energy of the primary beam through these foils, the energy of the beam seen by the detector was found to be $40.0 \mathrm{MeV}$. The lower energies of the $\alpha$ particles are obtained by putting standard aluminum absorbers in the beam.

A 1-mm-thick lithium-drifted silicon detector was used for this beam. The energy of the $\alpha$ particles that corresponds to the range in $1-\mathrm{mm}$ silicon is about $50 \mathrm{MeV}$. Hence this beam can be stopped in the detector. The spectral response of the energy absorbed in the detector from the corrected primary beam of $40 \mathrm{MeV}$ is shown in Fig. 2. The energy of the beam obtained from calibration is $39.88 \mathrm{MeV}$, which agrees very well with the corrected primary energy of $40 \mathrm{MeV}$. When a particle is stopped in a detector, the resolution is a function of the detector and the electronic system. Values for full width at half maximum (FWHM) from $2 \mathrm{keV}$ upwards (depending upon the type of experiment) have been obtained by many workers with semiconductor detectors exposed to monoenergetic particles, giving resolutions of much less than 1\%. FWHM obtained in this measurement is $0.62 \mathrm{MeV}$, giving a resolution of $1.5 \%$ (when the detector is operated at room temperature). This poorer resolution is partly due to momentum spread of the beam. However, better resolutions can be obtained by operating the detector at low temperature.

The spectra of different low-energy $\alpha$ particles obtained by degrading the primary beam by aluminum absorbers are also shown in Fig. 2, and the results are tabulated in $T$ abie 1. The agreement between the residual energies, calculated by using range-energy tables (5) and the experimental values, is good. The $\alpha$ particles emerging from the absorbing material, especially when the absorbers are thick, has a wider energy spread than the primary beam. This can be seen clearly in Fig. 2. This wider spread is due to small-angle scattering and ene rgy straggling.

BEAM: 910-MEV ALPHA PARTICLES The Berkeley 184-inch synchrocyclotron accelerates $\alpha$ particles to $910 \mathrm{MeV}$ and protons to $732 \mathrm{MeV}$. The response of a $0.48-\mathrm{gm} / \mathrm{cm}^{2}$ lithium-drifted silicon detector to $\alpha$ particles of $910 \mathrm{MeV}$ and lower energies was investigated. At high energies the detectors would operate as $\mathrm{dE} / \mathrm{d} \mathbf{x}$ detectors. The width of a peak is 


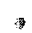

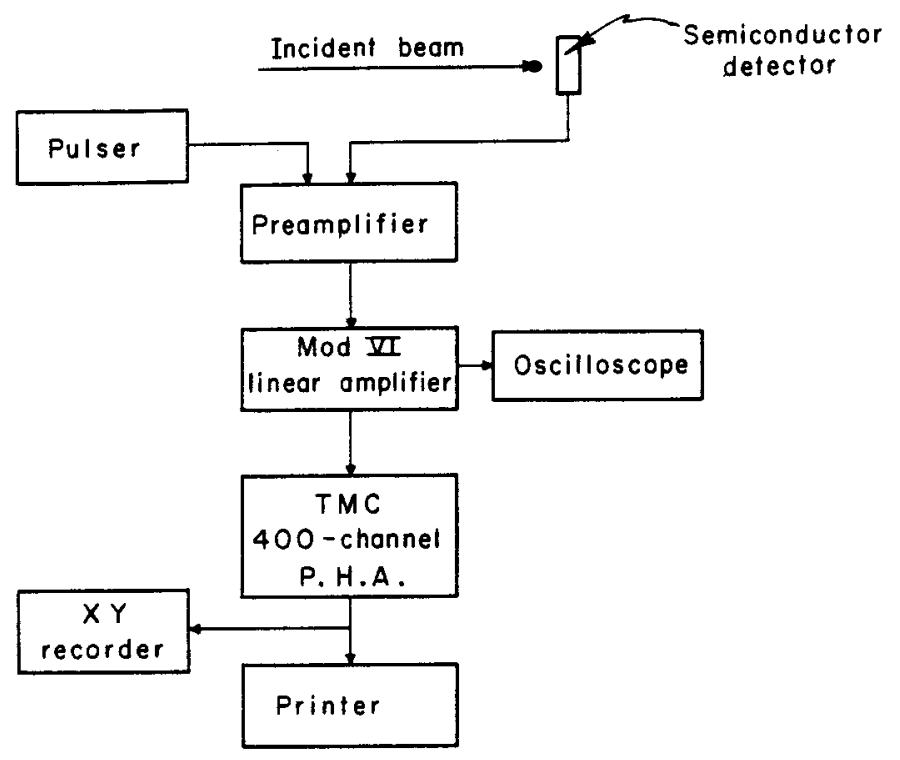

Figure 1. Experimental setup.

$M U B-4136 A$

Figure 2. Response of $1-\mathrm{mm}$ detector to $40-\mathrm{MeV} \alpha$ beam and the beam degraded by,

(a) 89.7 and $134.2 \mathrm{mg} / \mathrm{cm}^{2} \mathrm{Al}$;

(b) $155.2-\mathrm{mg} / \mathrm{cm}^{2} \mathrm{Al}$.

MUB-7539

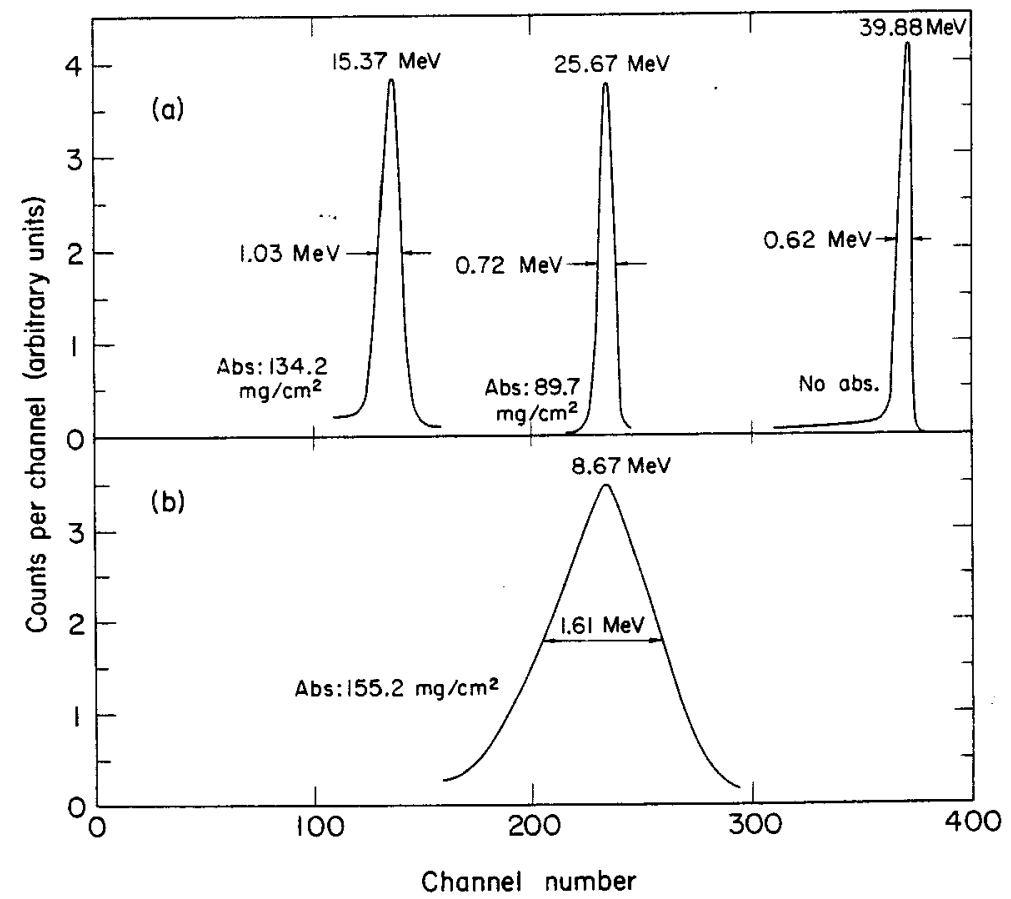


Table 1. 40-MeV $\alpha$ beam in 1-mm silicon detector

\begin{tabular}{ccc}
\hline Al absorber & \multicolumn{2}{c}{ Residual energy (MeV) } \\
\cline { 2 - 3 }$\left(\mathrm{mg} / \mathrm{cm}^{2}\right)$ & $\frac{\text { Calc. }}{40.00}$ & $\frac{\text { Expt. }}{39.88}$ \\
0 & 37.90 & 37.71 \\
15.2 & 35.71 & 35.67 \\
29.9 & 33.53 & 33.39 \\
44.5 & 31.16 & 31.01 \\
59.8 & 28.63 & 28.42 \\
75.0 & 25.96 & 25.67 \\
89.7 & 23.05 & 22.68 \\
104.5 & 15.59 & 15.37 \\
134.2 & 9.17 & 8.67 \\
155.2 & & \\
\hline \hline
\end{tabular}

determined by many factors in addition to those imposed by the electron system. Slight variations in path length, i. e., internal scattering, produce a spread in the energy absorbed. So do statistical fluctuations in the energy transfer in inelastic collision processes. Hence, particles of originally the same energy may produce measurably different signals, causing a spread of the energy-loss spectrum.

Since the charge collected from a detector is proportional to the los s of energy by the incident particle in the sensitive region, $\alpha$ particles, which are totally absorbed in this region, produce corresponding pulses of larger amplitude than $\alpha$ particles of higher energy, which pass completely through. For monoenergetic $\alpha$ particles whose range is greater than the depth of the depletion region, the charge-pulse amplitude from the detector increases with decreasing alpha energy until the range of $\alpha$ particles in silicon equals the sensitive depth. A further decrease in alpha energy causes a proportional decrease in the charge pulse.

The thickness of the detector depletion layer determines the measured energy loss. In our case, the depletion layer extended almost through the entire physical thickness of the detector, except for a few mils on the lithium side. This depletion thickness was measured by determining the maximum energy deposited in the detector. The range of the $\alpha$ particle in silicon whose energy corresponds to this maximum energy was then obtained from rangeenergy tables (5).

The response of the detector for the $910-\mathrm{MeV}$ beam and for the beam degraded by using copper absorbers is shown in Figs. 3 and 4. As we increased the thickness of the copper absorber in the beam, the energy of the $\alpha$ particles would be lowered and hence the $\mathrm{dE} / \mathrm{dx}$ would increase, with a concomitant increase in the energy spread. The experimental and theoretical values of energy loss shown in Table 2 are in close agreement down to a residual energy of $330 \mathrm{MeV}$. At lower energies, this agreement is lacking because at these energies the detector is not a $\mathrm{dE} / \mathrm{dx}$ detector. If we estimate the energy deposited in the detector at these lower energies, instead of $\mathrm{dE} / \mathrm{dx}$, a good agreement can be obtained. Experiments with detectors of thicknesses $1 / 4,1,3$, and $5 \mathrm{~mm}$ also gave good agreement with theoretical values 


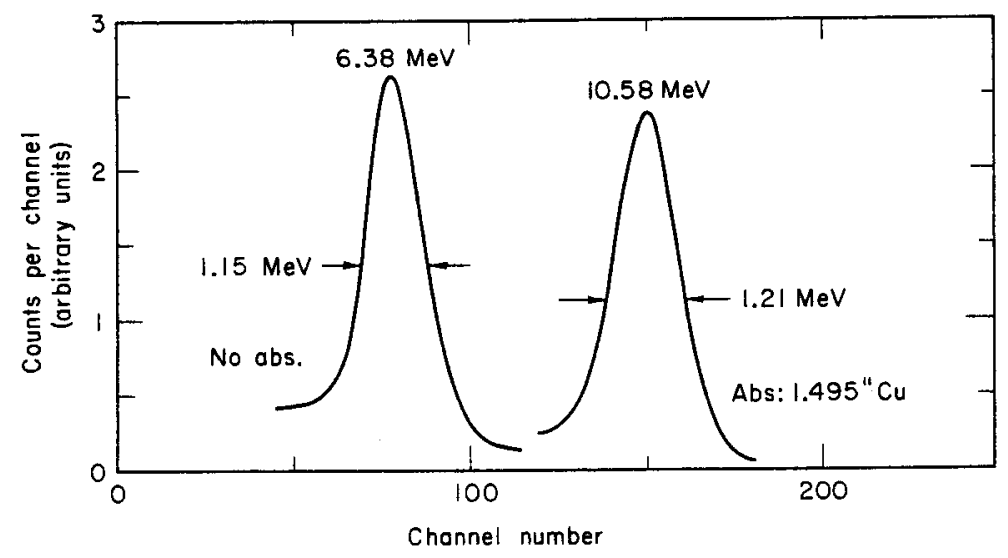

Figure 3. Response of $0.48-$ $\mathrm{g} / \mathrm{cm}^{2}(\approx 2 \mathrm{~mm})$ detector to $910-\mathrm{MeV} \alpha$ beam, and the beam degraded by $1.495 \mathrm{in}$. cu. MUB-7540

Figure 4. Response of 0.48 $\mathrm{g} / \mathrm{cm}^{2}(\approx 2 \mathrm{~mm})$ detector to $910-\mathrm{MeV} \alpha$ beam degraded by (a) 2.002 in. cu;

(b) 2.100 in. cu (Bragg peak). MUB-7541
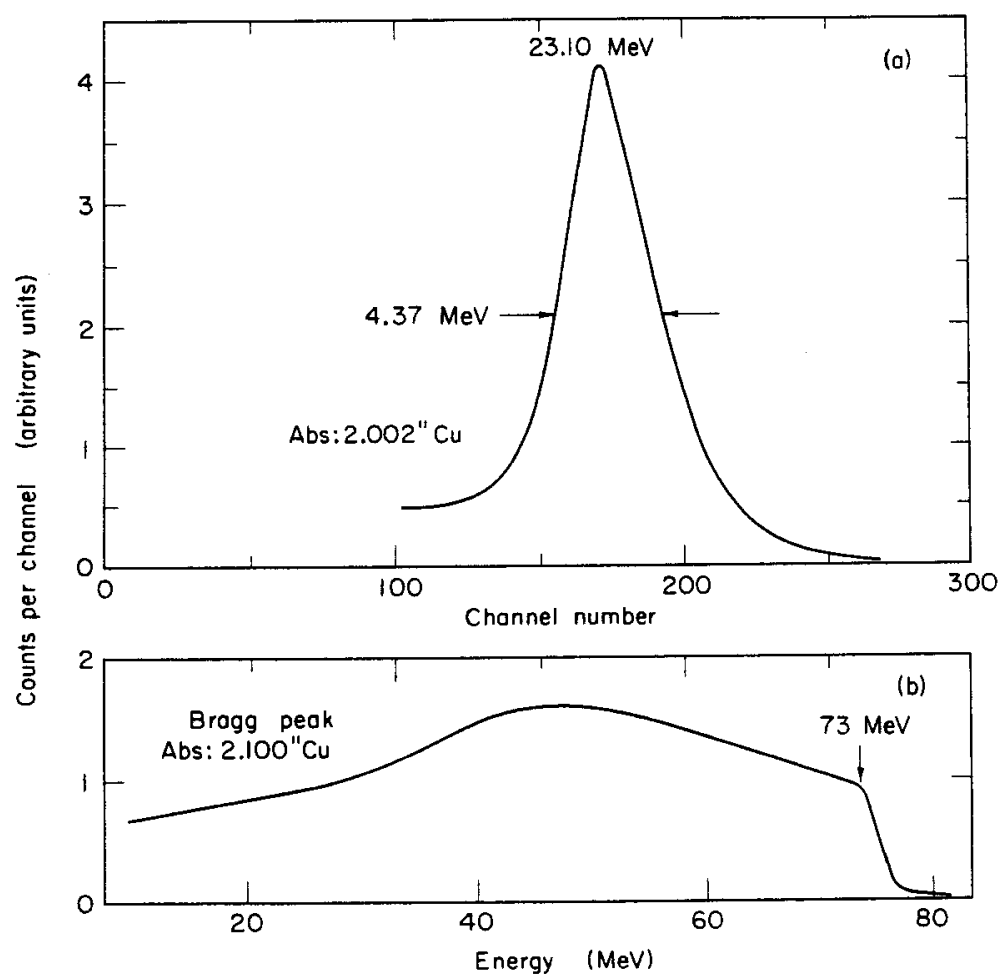
Table 2. 910-MeV $\alpha$ beam in $0.48-\mathrm{g} / \mathrm{sec}^{2}$ silicon detector

\begin{tabular}{|c|c|c|c|}
\hline \multirow{2}{*}{$\begin{array}{l}\text { Cu absorber } \\
\text { thickness } \\
\text { (in.) } \\
\end{array}$} & \multirow{2}{*}{$\begin{array}{c}\text { Residual } \\
\text { energy } \\
(\mathrm{MeV}) \\
\end{array}$} & \multicolumn{2}{|c|}{ Energy loss (MeV) } \\
\hline & & Theory & Expt. \\
\hline 0 & 910 & 6.44 & 6.38 \\
\hline 0.493 & 775 & 7.10 & 7.07 \\
\hline 1.001 & 620 & 8.25 & 8.28 \\
\hline 1.495 & 440 & 10.49 & 10.58 \\
\hline 1.755 & 330 & 12.92 & 13.35 \\
\hline 2.002 & 175 & 20.90 & 23.10 \\
\hline
\end{tabular}

of $\mathrm{dE} / \mathrm{dx}$. The Bragg peak obtained by use of ionization chambers was at $2.10 \mathrm{in}$. of copper. The response of the detector at the Bragg peak position is also shown in Fig. 4b. The maximum energy that can be deposited in the detector is from particles that have a range in silicon equal to the thickness of the depletion layer. In this experiment the maximum cutoff energy was $73 \mathrm{MeV}$. The corresponding range in silicon, from range-energy tables (5), is 0.48 $\mathrm{gm} / \mathrm{cm}^{2}$. The physical thickness of the detector as determined with a micrometer was about $5 \%$ greater than this value. This difference is due to the dead layer on the Li side. The experimental accuracy in the determination of detector thickness is within about $2 \%$.

It appears from these data that the energy spectrum of the $\alpha$ particles at the Braggpeak position extends to more than $75 \mathrm{MeV}$. This detector $\left(0.48 \mathrm{gm} / \mathrm{cm}^{2}\right)$ at this position was neither $d E / d x$ nor total-energy type. The small hump in Fig. $4 b$ is due to the particles of energy higher than $73 \mathrm{MeV}$ passing through the detector and hence depositing less energy. This hump vanished when the beam was degraded further by copper absorbers. As a rough estimate, a 1/4-mm-thick detector can be considered as $\mathrm{dE} / \mathrm{d} \mathbf{x}$ detector at the Bragg-peak position. The response of this detector at the Bragg-peak position is shown in Fig. 5. The energy loss in the detector was $4.8 \mathrm{MeV}$, FWHM $2.97 \mathrm{MeV}$. The energy of the alpha beam corresponding to this energy deposition is $80 \mathrm{MeV}$ and, at $50 \%$ level, 54 and $103 \mathrm{MeV}$. Thus we can roughly estimate that the energy spectrum of the alpha beam at the Bragg-peak position is mostly in the region 54 to $103 \mathrm{MeV}$, with an average value around $80 \mathrm{MeV}$.

The energy distribution at the Bragg-peak position can be obtained very accurately by using a detector thick enough to stop all the particles. A $5-\mathrm{mm}$-thick detector can stop $\alpha$ particles of energy $120 \mathrm{MeV}$. Figure 6 shows the energy distribution of $\alpha$ particles at the Bragg-peak position with a $5-\mathrm{mm}$-thick detector. It can be seen from the figure that the modal energy of the $\alpha$ particles at the Bragg position was $85 \mathrm{MeV}$. These data can be translated into LET distribution in water, which is shown in the same figure; the modal LET was around $10 \mathrm{keV} / \mu$.

BEAM: 49-MEV PROTONS Berkeley's 88-inch cyclotron accelerates protons up to $50 \mathrm{MeV}$ and $\alpha$ particles up to $120 \mathrm{MeV}$. Figure 7 shows the response of $0.68-\mathrm{gm} / \mathrm{cm}^{2} \mathrm{detector}$ for $49-\mathrm{MeV}$ protons. The energy loss in the detector is in good agreement with the theoretical value. The Bragg peak for the $49-\mathrm{MeV}$ proton beam was obtained at $2.58 \mathrm{gm} / \mathrm{cm}^{2}$ of aluminum. 


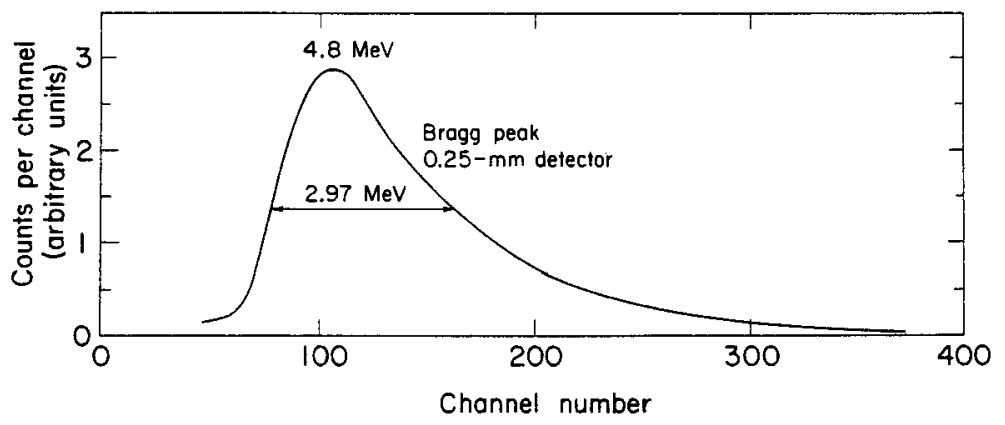

Figure 5. Response of $1 / 4-\mathrm{mm}$ detector to $910-\mathrm{MeV} \alpha$ beam at the Bragg-peak position.

MUB-7542

Figure 6. Distribution of $\alpha$ particle energy at the Braggpeak position of $910-\mathrm{MeV} \alpha$ beam.

MUB-7543

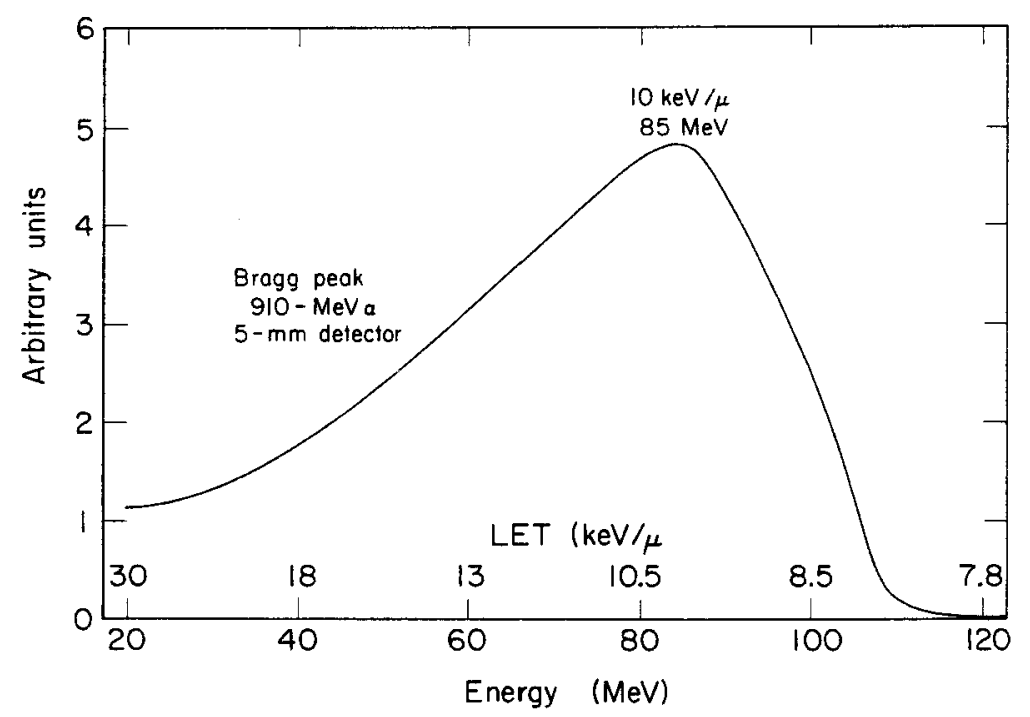




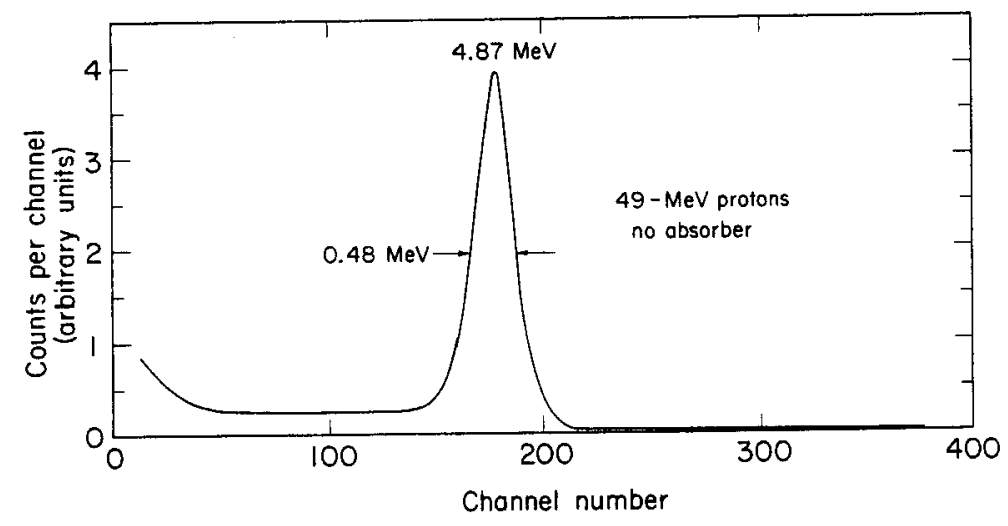

Figure 7. Response of 0.68 $\mathrm{g} / \mathrm{cm}^{2}(\approx 3 \mathrm{~mm})$ detector to 49-MeV proton beam.

MUB-7544

Figure 8. Energy distribution at the Bragg-peak position of 49-MeV proton beam. MUB-7545

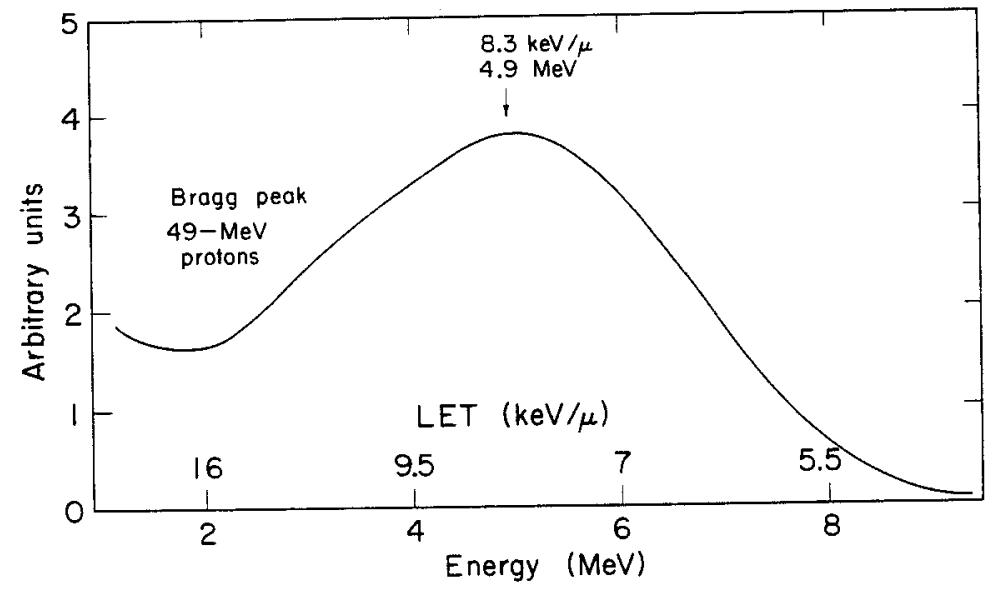


Figure 8 shows the energy distribution of protons at this Bragg-peak position. It can be seen from the figure that the modal energy of the proton is around $5 \mathrm{MeV}$. Corresponding LET values in water are also shown in the figure, the modal LET being around $8 \mathrm{keV} / \mu$.

\section{SUMMARY}

The lithium-drifted silicon semiconductor detectors used in this study of high-energy $\alpha$ particles and protons give very promising results in measuring energy loss and energy distribution. Indeed, the agreement between the theoretical and experimental values of residual energy and energy loss is very good. The energy distribution at the Bragg-peak positions of 910-MeV alpha beam and 49-MeV proton beam is measured in order to evaluate the LET distribution at the Bragg peak. From the data it seems that the modal energy of the heavy particles at the Bragg-peak position is roughly $10 \%$ of the primary beam energy. The fact that the particles have relatively high energies at the Bragg-peak position indicates that the LET there is not high. The rem dose at the downward slope of the Bragg peak, however, would be much higher than rad dose, because of the higher LET values there.

\section{ACKNOWLEDGMENTS}

I wish to thank Professor John H. Lawrence and Professor Cornelius A. Tobias for their encouragement. Special thanks to Donald A. Landis, Robert Lothrop and Harry E. Smith for supplying the detectors and for helpful discussions on their use. Also, the discussions with Fred Goulding, Dr. John T. Lyman, and Dr. Henry Aceto, Jr., are most appreciated.

This work was done under the auspices of the U. S. Atomic Energy Commission and the National Aeronautics and Space Administration.

\section{REFERENCES}

1. Goulding, F.; IEEE Trans. Nucl. Sci. NS-11, No. 3: 177-190, 1964.

2. Solid State Radiation Detectors, various articles in IRE Trans. Nucl. Sci. 8, Jan.,1961.

3. Raju, M. R.; Aceto, H., and Richman, C.; Pion Studies With Silicon Detectors, Nucl. Instr. and Methods, in press.

4. Maccabee, H. D. , and Raju, M. R.; Fluctuations of Energy Loss by Charged Particles in Silicon Detectors, Nucl. Instr. and Methods, in press.

5. Williamson, L., and Boujot, J. P.; Tables of Range and Rate of Energy Loss of Charged Particles of Energy 0.5 to $150 \mathrm{MeV}$, Centre d'Etudes Nucleaires de Saclay, 1962.

Received October, 1965. 


\title{
Studies of Vicia faba Root Meristems Irradiated with a $\pi$-Beam
}

\author{
Stephen P. Richman, Chaim Richman, Mudundi R. Raju and Bernard Schwartz
}

For some time we have been studying the dosimetric character of $\pi^{-}$beams produced by the Berkeley 184-inch cyclotron (1-3). Our interest stems from the fact that such a beam, if the intensity were sufficiently high, could have important applications in the radiotherapy of cancer $(1,4)$. The $\pi^{-}$-meson has the property that, at the end of its range, it is captured by a nucleus, causing the nucleus to explode into fragment s that contain a high proportion of shortrange, heavily ionizing $\alpha$ particles and protons. The dose distribution of such a beam in tissue-equivalent material gives a good ratio between the dose to the tumor and the dose to the intervening healthy tissue, even for deep-lying tumors. Of even greater importance is the fact that the high linear energy transfer (LET) of the particles in the tumor region might overcome some of the radio-resistance of an anoxic tumor.

The most useful beam available at the cyclotron for our purpose is a $90-\mathrm{MeV} \pi^{-}$

beam. At this energy the intensity is a maximum and the background is not excessive. The range of these pions is around $25 \mathrm{~cm}$ of tissue, which is long for the therapeutic situation. In studying the effects of the stopping pions, therefore, we had to use an appreciable amount of Lucite absorber, which not only attenuates the beam but also produces loss by divergence of the beam. These factors must be considered in irradiating biological materials. The dosimetry of this radiation presents a number of new problems that have not been entirely solved. Among these, at present, is that the background of electrons and muons in the beam amounts to about $40 \%$ of the particles. In spite of the questions that remain to be answered, it is useful to go ahead and look at some biological effects of pions. We have used the Vicia faba root meristem, since it is a simple system and a great deal of work has been done on the response of these cells to $x$-rays, $\gamma$-rays, and $\alpha$-particles.

Our technique has been to expose roots in the flat,or plateau,region and in the peak,or star,region of the $\pi^{-}$-meson "Bragg curve." The Bragg curve for these particles is not the usual Bragg curve that one obtains for a beam of $\alpha$ particles or protons. Because of the formation of stars at the end of the range the usual Bragg curve is augmented by the energy released in the medium by the star fragments. In determining this curve we have used two ionization chambers, one as a monitor and the second as a detector. The ionization in the peak, as measured by our chamber filled with one atm argon and carbon dioxide, must be considered as an approximation, since the fragments frequently have ranges much greater than the chamber so that wall effects are important. One advantage of the biological systems is that the medium receives the true dose from the fragments. 


\section{THE $\pi^{-}$BEAM AND EXPOSURE OF THE ROOT MERISTEMS}

Figure 1 shows the arrangement used for making the beam and irradiating the bean roots. The pions are produced by $732-\mathrm{MeV}$ protons striking a beryllium target. Since the pions are negative they are deflected away from the proton beam and leave the cyclotron tank through an appropriate window. A small quadrupole focusing magnet just inside the main concrete shielding helps to focus the pions. The beam then passes through a channel in the wall into the meson cave. When it reaches the cave the beam contains particles of various momenta and therefore a bending magnet is placed here which selects particles of $180 \mathrm{MeV} / \mathrm{c}$ to go into the quadrupole focusing magnet. The currents in the two sections of this focusing magnet can be set to focus the pions and also to control the shape of the beam according to the shape of the biological sample.

Before the roots are irradiated a careful run is made with varying thicknesses of Lucite absorber, from which we find the position of the augmented Bragg peak. (Figure 2 shows this curve taken for the second run to study the abnormal anaphases.) One set of bean roots is then placed in the plateau region and the other set in the peak region of the beam. The whole system is carefully lined up horizontally and vertically (with the aid of short exposures of Polaroid film) so that the root tips are irradiated, both in the peak and in the plateau region, by the full beam.

Figure 3 is a photograph of the bean roots in their water-filled Lucite boxes. In this run the beam passed through 2 in. of Lucite before it struck the plateau box, so that the beam would be uniformly spread out over the root meristems. In this particular case compressed air was bubbled through the boxes to guarantee good oxygenation. The box with the control roots is not shown in the photograph. The monitor chamber can be seen as well as the shielding and the cooling connections to the quadrupole focusing magnet. For the sake of convenience a Jordan dosimeter was put into the beam as the instantaneous monitor. This dosimeter has the meter connected to the sensitive chamber by a long pipe, and the meter was read by a closed-circuit TV system. Occasionally we maximized the beam by changing the position of the target in the cyclotron on the basis of this meter reading.

\section{THE RESPONSE OF THE Vicig faba ROOT MERISTEM TO IRRADIATION}

The Vicia faba root meristem has been chosen because it is not only extremely simple and economical, but, more important, it is sensitive to the low doses (100 to $400 \mathrm{R})$ that must be used. Obtaining even these doses requires about $20 \mathrm{hr}$ of irradiation because of the low dose rate. This paper reports the percentages of total anaphases that were abnormal at intervals after the end of the irradiation. Also the percentage of cells containing micronuclei has been scored at similar intervals (the root tips were stained with the orcein squash method). And thirdly, the growth rate after irradiation has been measured as a fraction of the control rate. Of these three effects of radiation, the growth-rate test is the simplest to perform. One simply raises a number of control and test roots, irradiates, and compares the average growth per day of the test roots with that of the control roots. In this case the average test increment per day is reported as a fraction of the average control increment for the same day. 


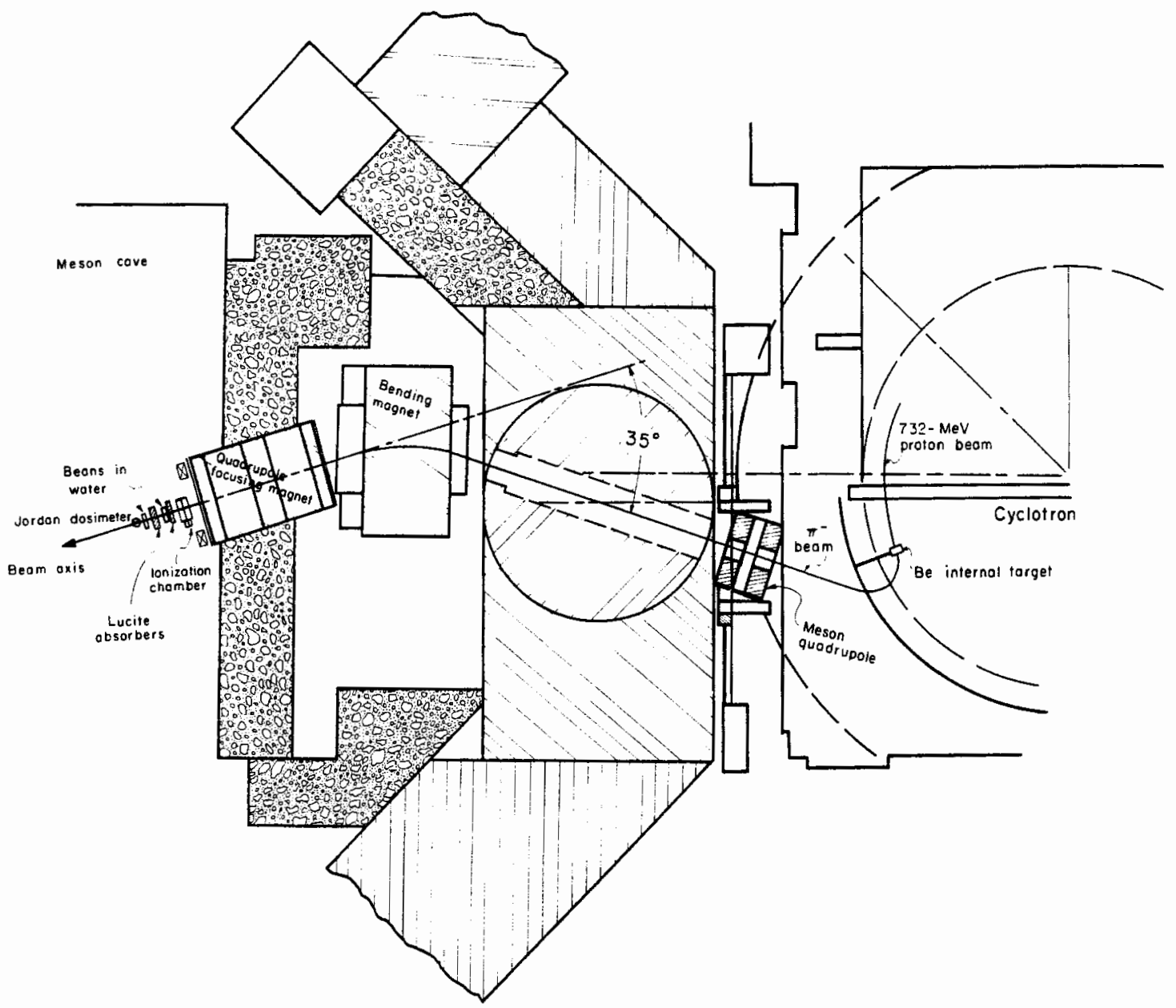

Figure 1. The $\pi^{-}$-mesons leave the target and are focused by a small quadrupole. They then go into the meson cave and there are deflected by the bending magnet and are focused by a second quadrupole. The shielding around the bending magnet and the second quadrupole helps to keep the background radiation low. 


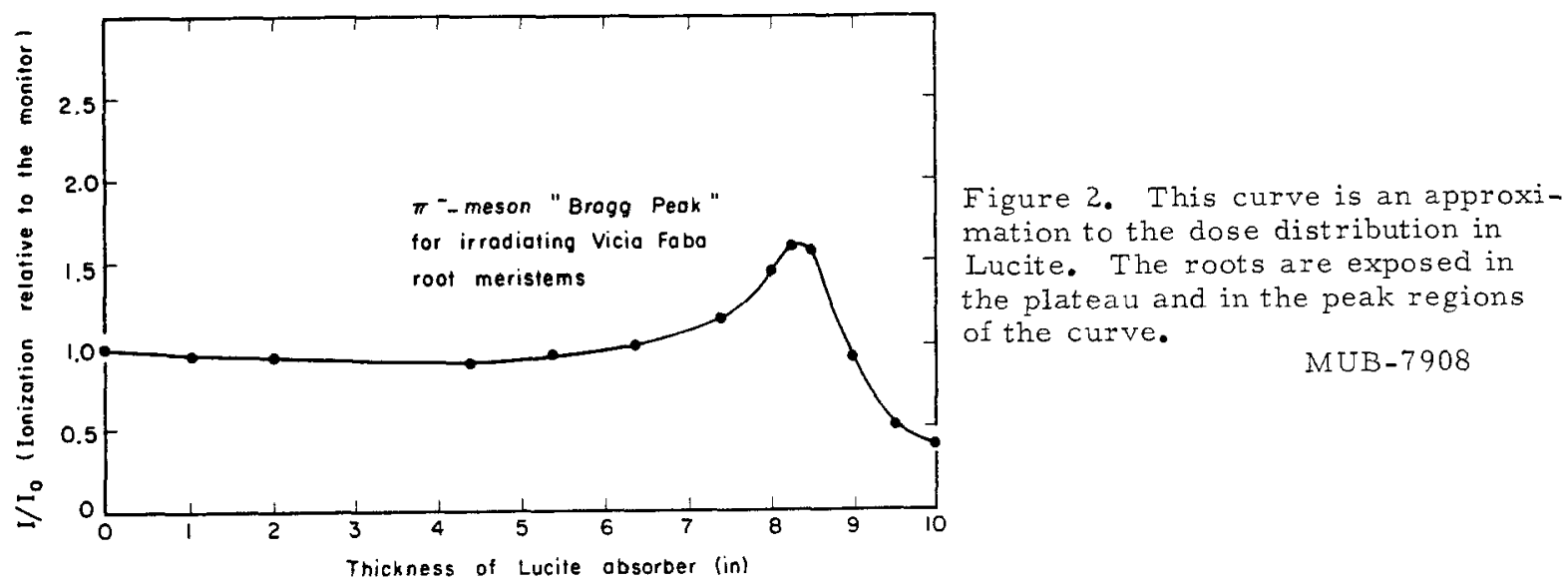

Figure 3. The photograph shows the arrangement of the beans in their boxes and the absorbers. Two monitors are used, an ionization chamber upstream and a Jordan dosimeter downstream. The cooling connections to the quadrupole focusing magnet can also be seen.

$Z N-5148$

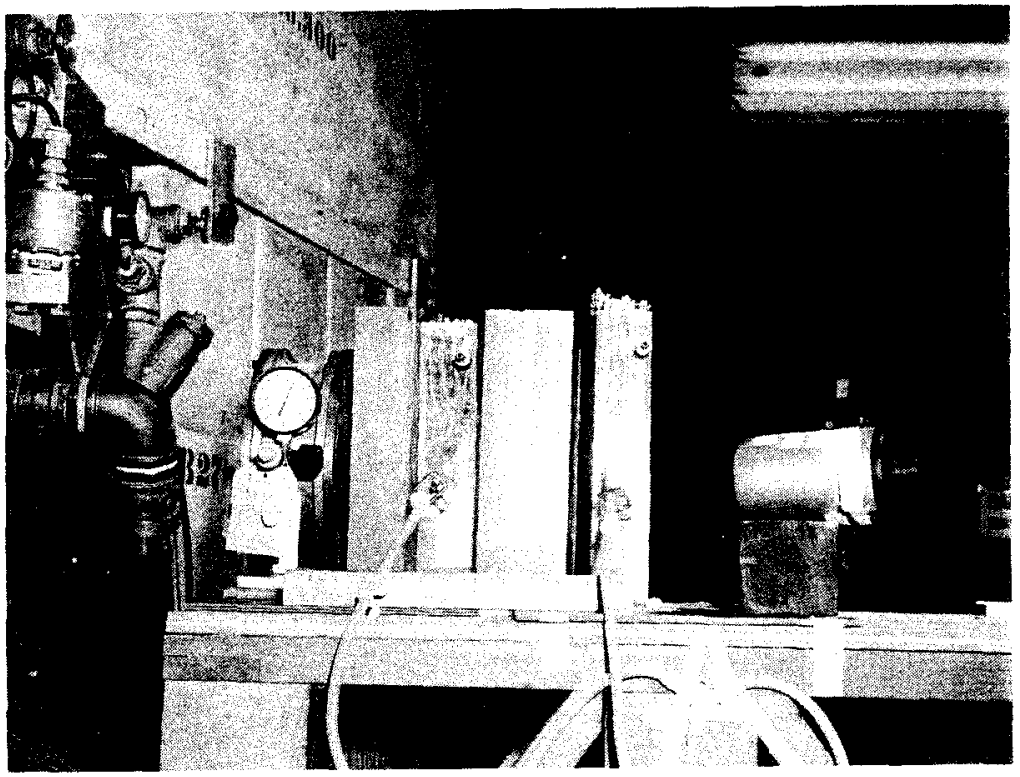


The literature on growth-rate tests employing Vicia faba is rather extensive. Of note here is the work by Gray and Scholes (5) with prolonged irradiations. One of the many problems caused by prolonged irradiation is that of recovery. That is, while receiving later phases of the dose, the material may still be recovering from the early phases. This recovery phenomenon or dose-rate dependence Gray and Scholes found to be pronounced for $X$ and $\gamma$ rays but nonexistent for $\alpha$ particles. For $\mathrm{X}$ and $\gamma$ rays, the growth rate dropped only half as much after chronic low exposure as after an acute dose of the same total amount, but the effect on growth rate was the same for $\alpha$ particles whether the dose was given over 10 min or $24 \mathrm{hr}$. This is of interest here because, in the plateau, pions produce ionization density much as do electrons. Therefore one should find a greater dose dependence in the plateau than in the peak position.

The primary cytological test reported here is the scoring of abnormal anaphases. For Vicia faba this method is very fast and simple, requiring little interpretation. One of the best uses to which anaphases have been put has been the work on the oxygen effect by Thoday and Read $(6,7)$, demonstrating that the effect of $X$ rays, but not of $\alpha$ particles, is sensitive to the amount of oxygen dissolved in the water containing the roots. It is hoped that the anaphase technique can demonstrate the same difference for pions, if the pions in the plateau region are substituted for $X$ rays, and the particles formed at the peak are substituted for the alphas. In Fig. 4 the word "anaphases" is in quotes because cells that are well into telophase have been included in the scoring. This is simply a count of the number of anaphases containing bridges and fragments expressed as a percentage of the total anaphases seen.

The fragments appearing at anaphase form micronuclei at telophase. These micronuclei remain in the cell for some time. For this paper, the number of meristem cells containing one or more micronuclei has been counted (and expressed as percent). Because micronuclei accumulate to some extent, micronuclei counts have proved convenient during the long fixation period to insure that no peaks in the anaphase percentage have been missed between fixation times.

The cell kinetics during a prolonged pion irradiation are only partly understood. Most important is the fact that, after an as yet unknown period of time, the cells in the meristem, both those irradiated at the peak and those at the plateau, stop dividing. This mitotic inhibition is due to the irradiation, and not to physical shock, since control tips placed nearby, but out of the beam, continue to divide. For this reason, much of the irradiation is carried out with the roots in interphase; and it is not until about $10 \mathrm{hr}$ after the end of the irradiation that division begins again. Both roots irradiated in the plateau and at the peak appear to resume division at roughly the same time.

\section{RESULTS}

Figure 4 shows the peak and plateau anaphase counts for one experiment. Each point represents at least two root tips. When division begins, both peak- and plateau-irradiated roots show the same percentage abnormality; but approximately the same peak-to-plateau ratio is maintained from 12.5 to $98 \mathrm{hr}$ after the irradiation. This ratio over all the points is 


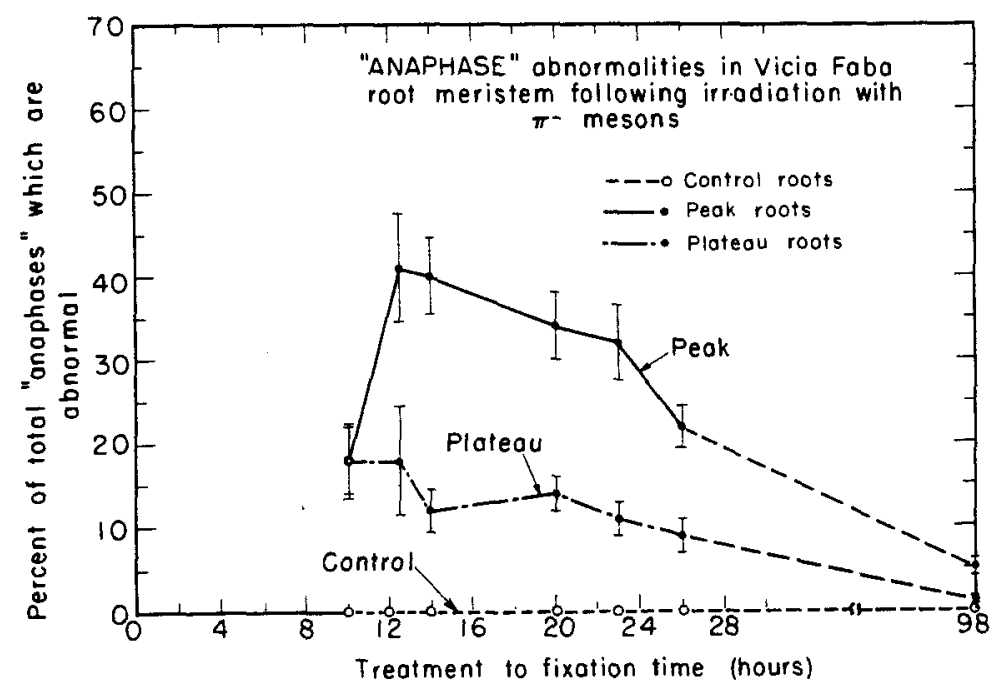

Figure 4. The count of abnormal anaphases for the roots irradiated in the peak and in the plateau and for the control roots as a function of fixation time after irradiation. The average is 2.2:1. A second experiment gave $2.6: 1$.

MUB-7407

Figure 5. The fraction of cells showing one or more micronuclei as a function of fixation time after irradiation. $M U B-7681$

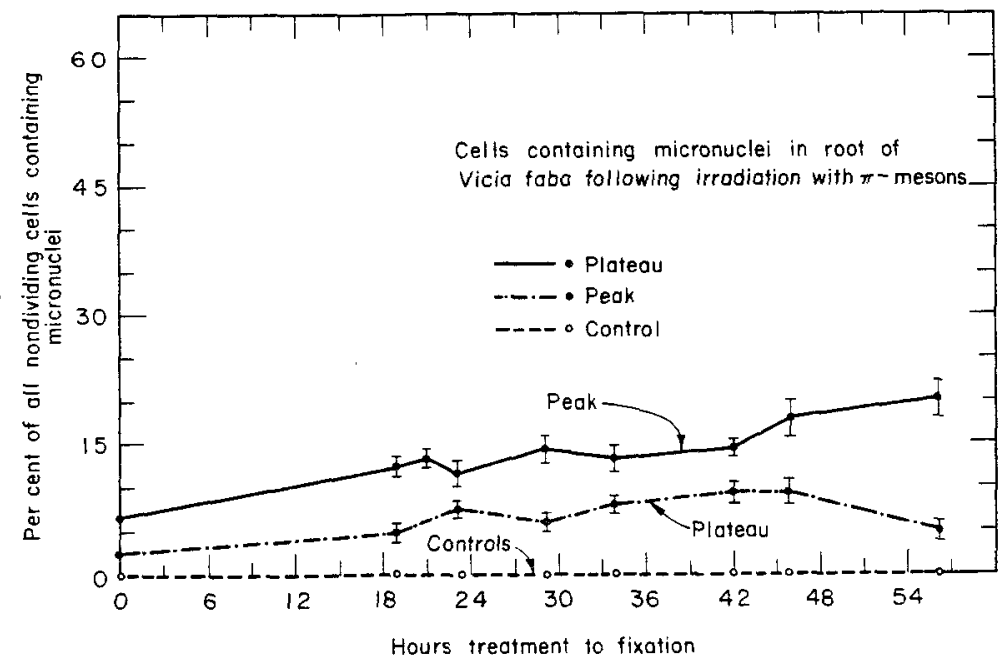




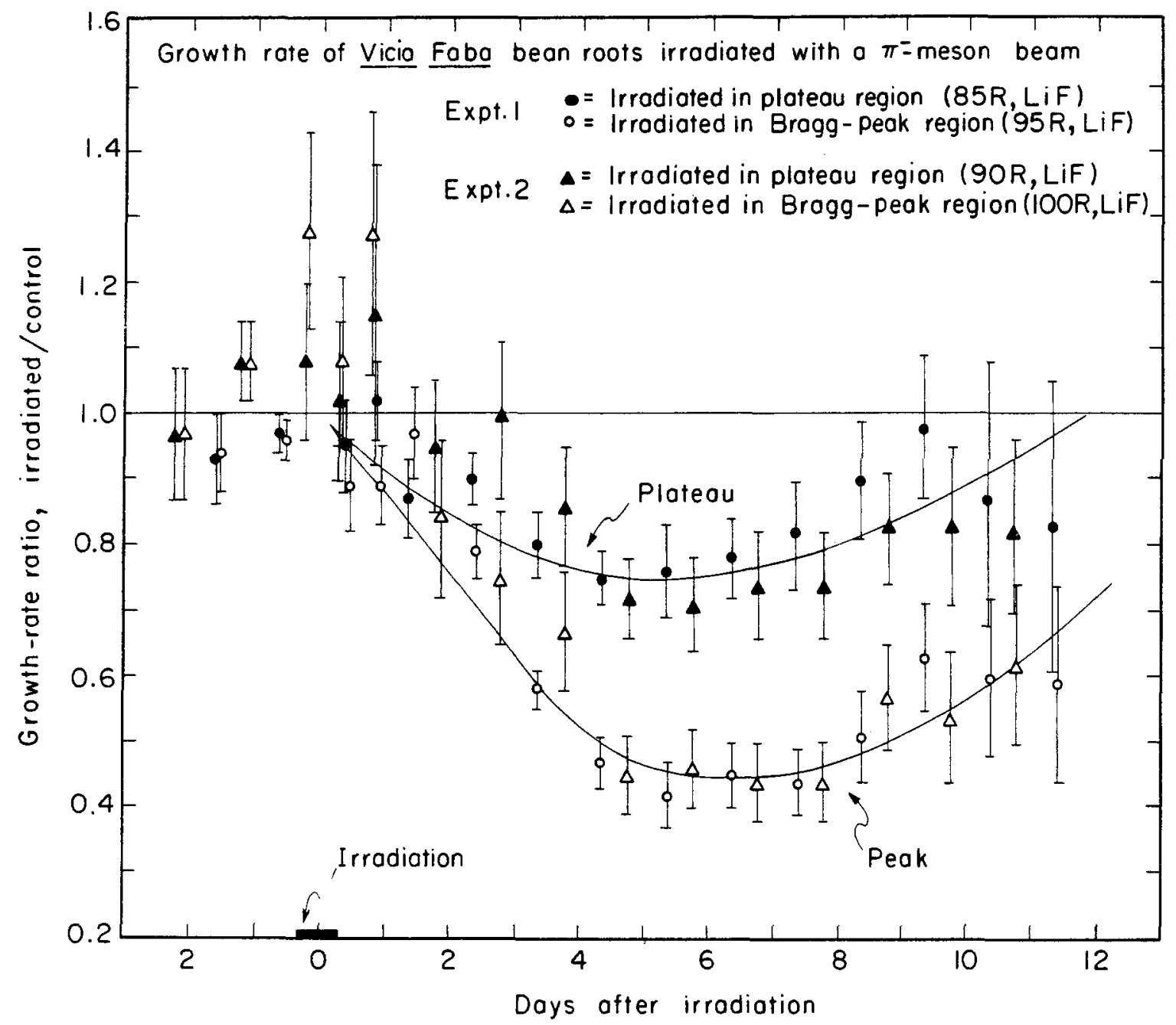

Figure 6. The growth rate per day of roots irradiated in the peak and in the plateau of the Bragg curve compared with control roots as a function of days after irradiation.

MUB-7568

2.2 to 1.0. The dose in the plateau region was about $100 \mathrm{R}$. A second irradiation at a higher dose (about $350 \mathrm{R}$ ) has given a ratio of 2.6 to 1.0 .

During the second irradiation, a count of the cells containing one or more micronuclei was taken. This is shown in Fig. 5. The micronuclei present at zero hours have resulted from cells that were in mitosis during the irradiation.

Figure 6 shows the results of two growth-rate experiments. About 15 roots were used in each box for each of these experiments. Six days after irradiation, the rate from peak irradiation has dropped to about 0.45 and that from the plateau to about 0.75 of the control rate. 
All three tests indicate that a significantly greater amount of damage has been received at the peak position. Although these data leave many questions unanswered, the results are very encouraging. About half of the plateau dose is due to contamination; when this is removed with an electrostatic separator, a much clearer picture of the effects of pions will be possible. In addition, the heavily ionizing particles in the star, particularly the alphas, are known to overcome the oxygen effect. Of interest then will be the irradiations in which the water in the boxes has been wholly or partially deoxygenated.

\section{ACKNOWLEDGMENTS}

It is a pleasure to express our thanks to Dr. John Lawrence and Dr. C. A. Tobias for their great interest, encouragement, and help in the course of this work. The cyclotron crew under the direction of Jimmy Vale was most helpful, not only in the operation of the machine but also with many problems of setting up the experiment. Dr. Henry Aceto, Jr., aided significantly with the growth-rate measurements. Catherine V. Richman was of constant help, night and day, with the staining of the root tips and scoring of the abnormalities.

Dr. Robert L. Thornton has given generous assistance and encouragement in the course of this entire work.

This work was carried out with the support of the U. S. Atomic Energy Commission and the American Cancer Society.

\section{REFERENCES AND NOTES}

1. Richman, C.; Aceto, H., Jr.; Raju, M. R.; Schwartz, B., and Weissbluth, M.; Semiannual Report, Donner Laboratory, Lawrence Radiation Laboratory, UCRL-11387, 1964.

2. Richman, C.; Aceto, H., Jr.; Raju, M. R., and Schwartz, B.; The Radiotherapeutic Possibilities of Negative Pions Preliminary Physical Experiments, Am. J. Roentzenol. Radium Therapy Nucl. Med., in press.

3. Raju, M. R.; Aceto, H., and Richman, C.; Pion Studies with Silicon Detectors, Nucl. Instr. Methods, in press.

4. Fowler, P. H., and Perkins, D. H.; Nature 189: 524-528, 1961.

5. Gray, L. H., and Scholes, M. E.; Brit. J. Radiol. 24: 285-291, 1951.

6. Thoday, J. M., and Read, J.; Nature 160:608, 1947.

7. Thoday, J. M. , and Read, J. ; Nature 163: 133, 1949.

Stephen P. Richman is at Medical School, Western Reserve University, Cleveland, Ohio.

Dr. Chaim Richman is professor of radiobiology at the Graduate Research Center, Dallas, Texas, and Department of Radiology, the University of Texas Southwestern Medical School, Dallas, Texas.

Received October, 1965 


\title{
Effect of Negative Pions on the Proliferative Capacity of Ascites Tumor Cells (Lymphoma L\#2) Grown In Vivo
}

\author{
José M. Foola, Chaim Richman, Mudundi R. Raju and John H. Lawrence
}

Negative pion beams produced by the 184-in. synchrocyclotron are studied with a view of determining their possible application in the treatment of cancer. The dosimetry of the pion beam $(1-4)$ as well as early results of radiobiological experiments $(5,6)$ have been reported. Preliminary studies on the effect of heavy particles on the proliferative capacity of ascites tumor cells grown in vivo have also been made in this laboratory (7-9). The present paper reports further investigation with an ascites lymphoma irradiated in mice and assayed in vivo.

\section{MATERIALS AND METHODS}

The pion beam is contaminated with negative muons and electrons. This contamination was found to be about $40 \%$ at the "plateau" (minimum-ionizing) portion of the beam and about 60\% at the "peak" (pion-stopping region) (1). The total ionization, as measured by ionization chambers at the pion-stopping region, was 1.3 times that at the plateau. The average total dose at plateau, as measured by LiF dosimeters (Co-60 calibration), was 150 rads.

Eight LAF $_{1}$ mice bearing a diploid ascites tumor (10) were irradiated in special cages, four at the plateau and four at the peak. Four animals were kept in identical enclosures as controls. Reference will be made to the animals and subsequent titrations according to their position in the cages. Figure 1 shows their position at plateau and peak with numbers given each animal. The same order holds for control mice. In all three groups, two animals were injected with $2 \times 10^{6}$ tumor cells three days prior to the experiment (mice number 1 and 2 in the three groups), and the other two with $10^{6}$ cells seven days prior to the experiment (mice number 3 and 4 in the three groups). These cells were supposedly more anoxic. Mice were fed ad lib. during the experiment. The irradiation was administered over a period of $34 \mathrm{hr}$ after which intraperitoneal fluid was withdrawn, cells were counted, serially diluted and injected into 510 adult females $\mathrm{LAF}_{1}$ recipient mice at various dilutions. Cells from both mice in the control group bearing three-day old tumor cells were pooled, counted, and serialIy injected I. P. The same was done with control mice bearing seven-day old tumor cells. For each of the mice irradiated at plateau and at peak, a group of LAF ${ }_{1}$ mice was used for tumor cell titrations in order to obtain the $L D_{50}{ }^{\prime} \mathrm{s}$. In all cases 10 animals per dilution were used except for mice 1 and 4 at peak and plateau, for which we used five animals per dilution. Proliferative capacity was evaluated at the end of eight weeks by the percentage of animals developing tumors $(7,8,9)$. $L D_{50}$ 's (the number of cells necessary to produce tumors or death in $50 \%$ of the animals) and standard deviations were calculated by the methods of 


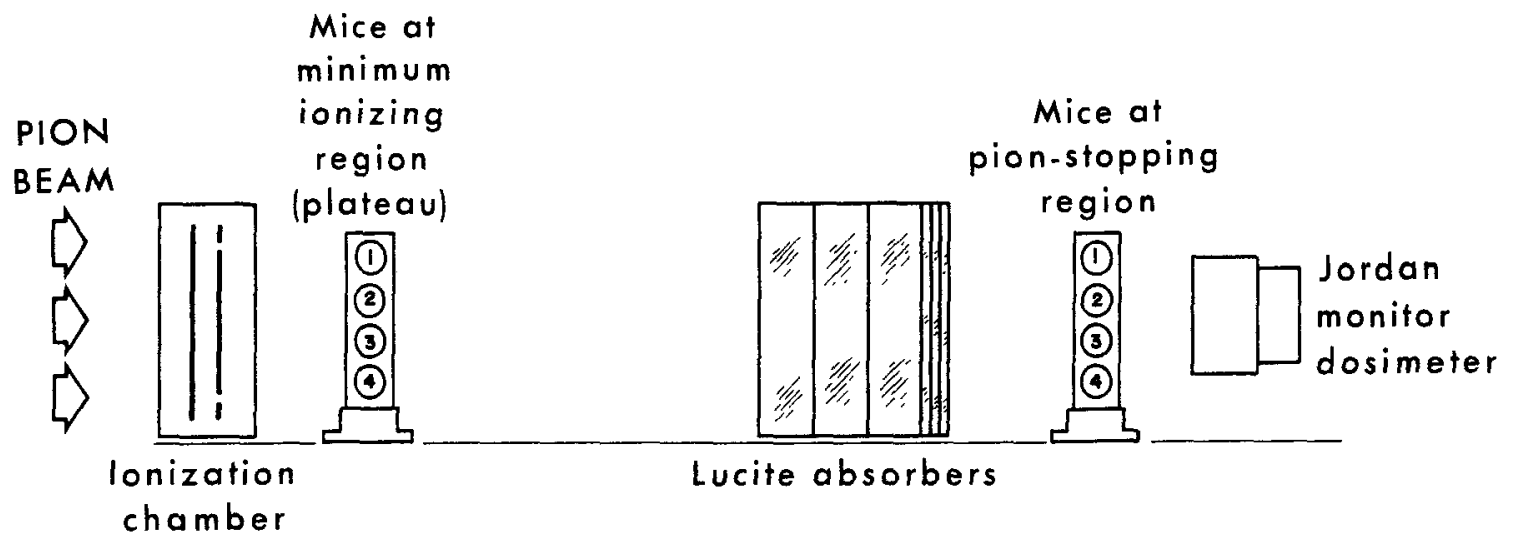

Figure 1. Schematic representation of experimental set-up, showing position of mice during irradiation. MU B-96OC

Litchfield and Wilcoxon (11), and surviving fractions were calculated by comparison with control groups.

\section{RESULTS}

Table 1 shows the results obtained. $L D_{50}$ 's are given with their standard deviations, which are asymmetric because of the logarithmic dilution procedure and the use of log-normal plots in the statistical method. Surviving fractions (S. F.) have been calculated by comparison with the proper control group. The errors in the control $\mathrm{LD}_{50}$ 's have been propagated in the calculation of surviving fractions.

\section{DISCUSSION}

No clear conclusions can be drawn from the experimental data shown in Table 1. The average of the surviving fractions for three-day ascites cells are:

$$
\begin{array}{rr}
\text { For PL } 1 \text { and 2: } & \text { S.F. }=0.160 \begin{array}{r}
+0.676 \\
-0.128
\end{array} \\
\text { For PK } 1 \text { and 2: } \quad \text { S.F. }=0.029+0.107 \\
-0.025
\end{array}
$$

and for seven-day old cells:

$$
\begin{aligned}
& \text { For PL } 3 \text { and 4: S.F. }=0.129+0.154 \\
& \text { For PK } 3 \text { and 4: S.F. }=0.032+0.105
\end{aligned}
$$

And if the four values of the surviving fraction at plateau and at peak are averaged:

$$
\begin{aligned}
\text { S.F.PL }=0.140 \begin{array}{l}
+0.554 \\
-0.113
\end{array} \\
\text { S.F.PK }=0.0305^{+0.1056}-0.0245
\end{aligned}
$$

The ratios of these surviving fractions, without considering the errors, are:

$$
\begin{aligned}
& S . F \cdot{ }_{P L} / S \cdot F \cdot{ }_{P K 3}=5.5 \\
& S . F \cdot P_{P L} / S \cdot F \cdot{ }_{P K 7}=4.0
\end{aligned}
$$


and for the averages:

$$
\text { S. F.PL } / \mathrm{S} \cdot \mathrm{F} \cdot \mathrm{PK}=4.6
$$

Table 1. $\mathrm{LD}_{50}$ 's and surviving fractions for ascites tumors irradiated in mice

Mice injected three days before exposure

\begin{tabular}{|c|c|c|c|}
\hline Group & $\mathrm{LD}_{50} \pm \mathrm{SD}$ & S. F. $\pm S D$ & $\begin{array}{c}\text { Total Dose } \\
\text { (Rads) }\end{array}$ \\
\hline Control & $\begin{array}{l}+2.24 \\
1-0.41\end{array}$ & $\equiv 1$ & \\
\hline Plateau 1 & $\begin{array}{r}3.5+7.4 \\
-1.4\end{array}$ & $0.286+1.244$ & $150 \pm 30$ \\
\hline Plateau 2 & $\begin{array}{r}30+30 \\
-10\end{array}$ & $\begin{array}{r}0.033+0.108 \\
-0.025\end{array}$ & $150 \pm 30$ \\
\hline Peak 1 & $\begin{array}{r}25+38 \\
-13\end{array}$ & $0.040^{+0.150}=0.037$ & $195 \pm 45$ \\
\hline Peak 2 & $56 \begin{array}{l}+71 \\
-20\end{array}$ & $\begin{array}{l}0.0179+0.0626 \\
-0.0137\end{array}$ & $195 \pm 45$ \\
\hline
\end{tabular}

Mice injected seven days before exposure

\begin{tabular}{|c|c|c|c|}
\hline Control & $\begin{array}{r}+5.00 \\
2-0.85\end{array}$ & $\equiv 1$ & \\
\hline Plateau 3 & $\begin{array}{l}50+56 \\
-17\end{array}$ & $\begin{array}{r}0.040+0.145 \\
-0.031\end{array}$ & $150 \pm 30$ \\
\hline Plateau 4 & $\begin{array}{c}10^{+11} \\
-4\end{array}$ & $\begin{array}{r}0.200+0.720 \\
-0.165\end{array}$ & $150 \pm 30$ \\
\hline Peak 3 & $\begin{array}{r}35+27 \\
-11\end{array}$ & $0.0571^{+}+0.1870$ & $195 \pm 45$ \\
\hline Peak 4 & $280+200$ & $0.00714_{-0.00515}^{+0.02300}$ & $195 \pm 45$ \\
\hline
\end{tabular}

This peak to plateau ratio of the surviving fraction suggests the greater effectiveness of the peak radiation over the plateau radiation. The dose at the peak is estimated to be only $1.3 \pm 0.3$ times the plateau dose, in other words, $195 \pm 45$ rads. Such small difference between the peak dose and the plateau dose would not seem to explain the greater effectiveness shown by a ratio of 4.6. This result is supported by a related experiment on chromosome aberrations produced in irradiated root meristems of Vicia faba (6). Also, a higher frequency of polyploid cells and "anaphase bridges" produced at the pion-stopping region as compared to the one produced at plateau has been reported (5), and a confirmatory experiment was done simultaneously with the same animals and cells used in the present work (12). The doses at plateau and peak being nearly the same, it would seem that the difference found in surviving fractions is due to a greater $\mathrm{RBE}$ of the pion-stopping region (or 'star' region) as compared to the plateau region. 


\section{ACKNOWLEDGMENTS}

We wish to express our appreciation to Alice Beckmann and Henriette Cozza for their technical assistance in performing this experiment.

\section{REFERENCES}

1. Richman, C.; Aceto, H.; Raju, M. R., and Schwartz, B.; The Therapeutic Possibilities of Negative Pions, Preliminary Physical Experiments, to be published in Am. $J$. Roentgenol.

2. Richman, C.; Aceto, H.; Raju, M. R.; Schwartz, B., and Weissbluth, M.; Semiannual Report, Donner Laboratory, Lawrence Radiation Laboratory, UCRL-11387: 114-126, 1964.

3. Raju, M. R.; Aceto, H., and Richman, C.; Semiannual Report, Donner Laboratory, Lawrence Radiation Laboratory, UCRL-16246: 34-99, 1965; Nucl. Instr. Methods 37: 152$158,1965$.

4. Aceto, H.; Ph. D. Thesis, University of California, Lawrence Radiation Laboratory Report, UCRL-11482, 1964.

5. Loughman, W. D.; Winchell, H, S.; Aceto, H.; Richman, C. ; Raju, M. R., and Lawrence, J. H. ; Semiannual Report, Donner Laboratory, Lawrence Radiation Laboratory, UCRL-16246: 100-102, 1965.

6. Richman, S.; Richman, C.; Raju, M. R., and Schwartz, B.; this report, see p. 15.

7. Sillesen, K.; Lawrence, J. H., and Lyman, J. T.; Semiannual Report, Donner Laboratory, Lawrence Radiation Laboratory, UCRL-11033: 139-151, 1963.

8. Schmidlin, Y.; Lawrence, J. H.; Sillesen, K.; Welch, G., and Lyman, J.; Semiannua1 Report, Donner Laboratory, Lawrence Radiation Laboratory, UCRL-11833: 80-94, 1964.

9. Sillesen, K.; Lawrence, J. H., and Lyman, J. T. ; Acta Isotopica 3: 107-126, 1963.

10. Amos, D. B.; Ann. N. Y. Acad. Sci. 63: 706-710, 1956.

11. Litchfield, J. T., Jr., and Wilcoxon, F.; J. Pharm. Expt1. Therap. 96: 99-113, 1949.

12. Loughman, W. D. sand Winchell, H. S.; personal communication.

Received December, 1965. 


\title{
Effect of $\mathrm{NaClO}_{4}$ on Bacteriophage: Release of DNA and Evidence for Population Heterogeneity
}

\author{
David Freifelder
}

One of the many problems involved in the isolation of DNA from bacteriophages is possible attack by trace amounts of nucleases that have survived the procedures used for purification and release. In an investigation of the denaturation of DNA in concentrated solutions of $\mathrm{NaClO}_{4}$, Hamaguchi and Geiduschek (1) noted that proteins contaminating the DNA samples were precipitated in the $\mathrm{NaClO}_{4}$ solution. This suggested the possibility that if the DNA were isolated in the presence of high concentrations of $\mathrm{NaClO}_{4}$, such nucleases might be inactivated. However, when in preliminary experiments phages were placed in $5 \underline{\mathrm{M}} \mathrm{NaClO}_{4}$ in preparation for DNA extraction, a rapid increase in viscosity suggested that DNA release had already occurred in the $\mathrm{NaClO}_{4}$ solution. In this paper, the characteristics of the reaction between $\mathrm{NaClO}_{4}$ and phages, and the properties of the DNA released in this reaction will be described. In short, it has been found that (i) nucleic acids can be extracted in this way probably from all phages and from many animal and plant viruses, (ii) phage populations are heterogeneous in their response to $\mathrm{NaClO}_{4}$, and (iii) by controlling the reaction conditions, the DNA can be released either free in solution or attached in an, as yet unknown, way to the phage protein coat. A preliminary report of this procedure has already appeared, Freifelder (2).

\section{MATERIALS AND METHODS}

Preparation and purification of phages $T 7 M, T 40^{r}$ and $B 3$ have been described, Davison, Freifelder, and Holloway (3). B. subtilis phage SP50 was a gift of Dr. T. Trautner. The basic techniques of ultracentrifugation with UV optics are those used previously, Davison and Freifelder (4). All sedimentation coefficients are corrected for viscosity and temperature (and are therefore $S_{20, w}$ ) and are for $20 \mu \mathrm{g} / \mathrm{ml} \mathrm{DNA}, 30 \mathrm{~mm} \mathrm{Kel}-\mathrm{F}$ cell, and $33,450 \mathrm{rpm}$.

The $\mathrm{NaClO}_{4}$ stock (Fisher: "purified" grade) was prepared as follows: A 7.4 molar solution was made in $0.002 \underline{M}$ versene, $\mathrm{pH}$ 7.5. One molar $\mathrm{NaOH}$ was added dropwise until a 40-fold dilution of the solution had a pH of ca. 7.5 (pH meter). This solution was stored at $4^{\circ} \mathrm{C}$. After about one month, the pH (40-fold diluted) had decreased to less than 6; associated with this was inefficient DNA release. The activity of the solution could be restored by addition of $\mathrm{NaOH}$ although usually the solution was discarded.

The basic procedure for DNA release was the following: 5 to $10 \mu \mathrm{l}$ of a concentrated phage suspension $\left(\mathrm{OD}_{260}>50\right)$ was added to $150 \mu \mathrm{l}$ of $\mathrm{NaClO}_{4}$ at the desired concentration, obtained by dilution of the $7.4 \mathrm{M}$ stock with $0.01 \underline{\mathrm{M}}$ sodium $\mathrm{PO}_{4}, \mathrm{pH} 7.8,-0.001 \underline{\mathrm{M}}$ versene, $\mathrm{pH}$ 7.8. After incubation at room temperature for various times, $1.2 \mathrm{ml}$ of a NaCl stock was 
added to give a final $\mathrm{Na}$ concentration of one molar for centrifugation. In order to determine the percentage of DNA released in a given sample, the solution was centrifuged and photographed in the Spinco Model E analytical ultracentrifuge for $15 \mathrm{~min}$ at 8,000 to 12,000 rpm followed by $15 \mathrm{~min}$ at 33,450 . The UV photograph was traced with a Joyce Loebl Recording Microdensitometer, and from the relative heights of the phage and DNA boundaries, the relative percentages of phage and of DNA were calculated.

A note of caution: The $\mathrm{K}, \mathrm{Cs}, \mathrm{Mg}$, and $\mathrm{Ca}$ perchlorate salts are insoluble.

\section{RESULTS}

EFFECT OF $\mathrm{NaClO}_{4}$ ON VIABILITY Figure 1 shows the loss of viability of $\mathrm{T} 7$ following brief incubation in fairly low concentrations of $\mathrm{NaClO}_{4}$. Such curves are not easily reproducible because of the very rapid initial decrease in titer. For each perchlorate concentration, there is a small fraction that is relatively resistant to inactivation. To see if this is a genotypic property, plaques from surviving phage were picked and lysates prepared from them. These phages upon reincubation in $\mathrm{NaClO}_{4}$ were as sensitive as the original culture so that this resistance is not a genetic property of the phage. Similar experiments have been done with T 4 and B3. To determine the cause of this inactivation, T4 and T7 were treated with $1 \mathrm{M} \mathrm{NaClO}$ for $1 \mathrm{~min}$, diluted to $0.1 \mathrm{M}$ and mixed with sensitive bacteria. After $5 \mathrm{~min}$ at $37^{\circ} \mathrm{C}$ the bacteria were spun out at $2,000 \mathrm{~g}$ for $5 \mathrm{~min}$. The supernatant was then examined in the ultracentrifuge. A sedimenting boundary characteristic of whole phage particles was found, suggesting that inactivation in $1 \mathrm{M} \mathrm{NaClO}_{4}$ is a result of loss of ability to adsorb to host bacteria. Phage T4 so treated was also examined in the electron microscope using negative staining (Brenner et al. (5)), and it was found that the tails are contracted, which is consistent with the los of ability to adsorb. Similar results obtain for phage SP-50 when inactivated in $0.1 \mathrm{M} \mathrm{NaClO}_{4}$ (Trautner and Kleinschmidt, personal communication).

DNA RELEASE BY HIGH CONCENTRATIONS OF $\mathrm{NaClO}_{4}$ As reported earlier, incubation for brief periods (10 sec to $10 \mathrm{~min}$ ) in $5.1 \mathrm{M} \mathrm{NaClO}_{4}$ results in release of nucleic acid by coliphages T4, T7 and $\lambda$; Pseudomonas phage B3; B. subtilis phage SP-50; Hemophilus influenzae phage HP-1 (C. S. Rupert, personal communication); E. coli male-specific RNA phage MS-2 (kindly provided by Drs. H. Fraenkel-Conrat and J. Tung); tobacco mosaic virus (gift of Dr. H. Fraenkel-Conrat); and the animal viruses, Shope papilloma and reovirus (A. K. Kleinschmidt, personal communication). In the following, the results with phages T7, $\mathrm{T} 4$. and $\mathrm{B} 3$ will be given in detail.

STUDIES WITH E. coli PHAGE T7M Figure 2a shows the sedimentation boundary for I7 DNA released by incubation of the phage for $1 \mathrm{~min}$ in $5.1 \mathrm{M} \mathrm{NaClO} 4^{*}$ The boundary is sharp with no trailing material and characteristic of unsheared $T 7$ DNA (4). DNA released by $\mathrm{NaClO}_{4}$ is comparable to $\mathrm{T} 7 \mathrm{DNA}$ released by heating for 5 min at $70^{\circ} \mathrm{C}$ ir $0.2 \mathrm{M} \mathrm{NaCl}$. When perchlorate- or heat-released $T 7$ DNA are mixed together or sedimented separately in either $1 \underline{\mathrm{M}} \mathrm{NaCl}, 1 \underline{\mathrm{MaClO}} 4$, or $0.5 \mathrm{M} \mathrm{NaCl}-0.5 \mathrm{M} \mathrm{NaClO}_{4}$, the boundaries are indistinguishable with respect to boundary shape and sedimentation coefficient $\left(\mathrm{S}_{20, \mathrm{w}}=29\right.$ at $20 \mu \mathrm{g} / \mathrm{ml}, 33,450$ rpm). Perchlorate-released DNA was also heated to $70^{\circ} \mathrm{C}$ (after dilution) for 5 min with no 


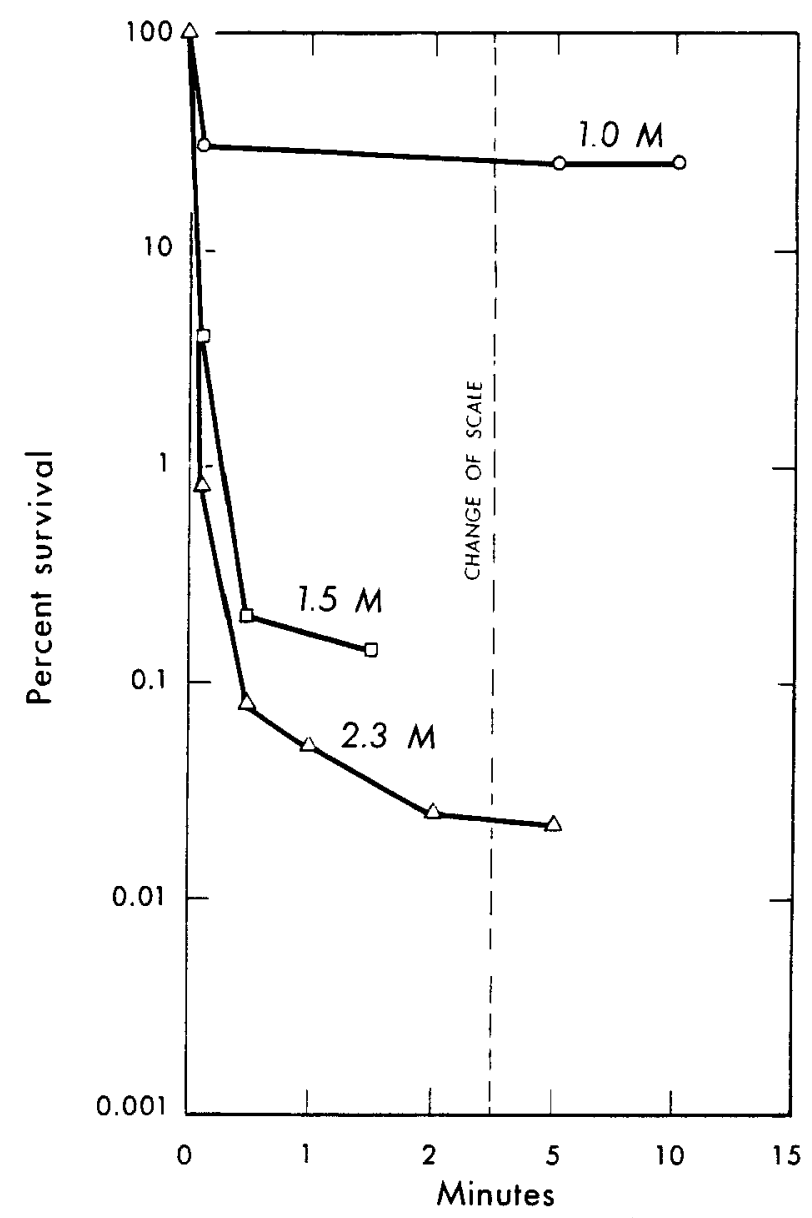

Figure 1. Loss of viability of $\mathrm{T} 7$ as a function of time of incubation in various concentrations of $\mathrm{NaClO}_{4}$. $0.1 \mathrm{ml}$ phage was rapidly mixed with $1.0 \mathrm{mI} \mathrm{NaClO}$ to obtain the indicated concentrations. The reaction was stopped by a 100-fold dilution into $0.01 \mathrm{M} \mathrm{PO}_{4}, \mathrm{pH} 7.8$. MUB-7516

Figure 2. Photometric trace of an ultraviolet absorption photograph of the sedimentation distribution diagram for (a) native and (b) denatured T7 DNA. In (a) phages were added to $5.1 \mathrm{M} \mathrm{NaClO}$ and diluted five-fold $1 \mathrm{~min}$ later. In (b) DNA was released in $5.1 \mathrm{M} \mathrm{NaClO}$, diluted, and denatured by addition, in order, of $1 / 2$ volume $1 \mathrm{M} \mathrm{NaOH}$, $1 / 2$ volume $9 \% \mathrm{HCHO}$, and $1 / 12$ volume $1 \mathrm{M} \mathrm{NaH}_{2} \mathrm{PO}_{4}$. Conditions of centrifugation - 33,450 rpm. $30 \mathrm{~mm} \mathrm{Kel-F}$ cell. The meniscus is at $M$; direction of sedimentation is indicated by the arrow.

MUB-7517

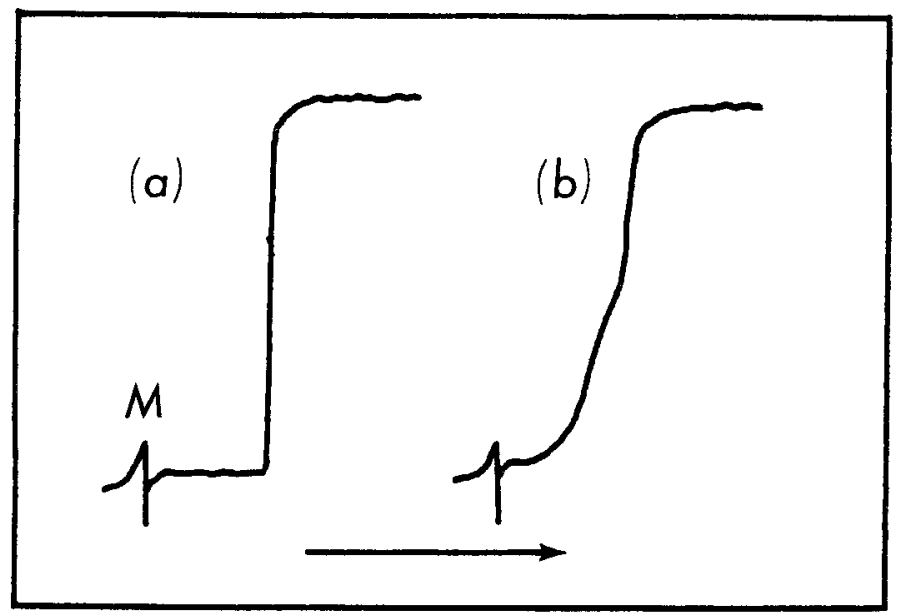


detectable effect on the boundary. Perchlorate-released DNA was also shown to be native (undenatured) since

1. there is no detectable spectral shift by addition of $\mathrm{HCHO}$,

2. boiling the DNA produces the maximum (32\%) hyperchromic effect, and

3. heating in HCHO produces $45 \%$ hyperchromicity.

T 7 DNA samples released by perchlorate were denatured with alkali or by heating in the pres ence of $12 \%$ HCHO and compared with heat-released DNA that was similarly treated. The sedimentation boundaries were in all cases indistinguishable; that is, in agreement with earlier work (Freifelder and Davison (6)), 50\% of the material sedimented as a sharp front with $\mathrm{S}_{20, \mathrm{w}}=29$ and $50 \%$ moved as a broader, slower boundary (Fig. $2 \mathrm{~b}$ ). Hence, the perchlorate extraction procedure does not introduce single strand breaks into the DNA, Davison, Freifelder and Holloway (3).

The possibility that phage with partially released DNA might exist as an intermediate in the release process was then investigated by incubating in $\mathrm{NaClO}_{4}(2.3,3.7$, or 5.1 molar) for various periods of time prior to dilution to 1.0 molar. In 1.0 molar $\mathrm{NaClO}_{4}$, T7 is stable for $24 \mathrm{hr}$ (although viability is rapidly lost; see Fig. 1). By centrifuging the solution first at $12,590 \mathrm{rpm}$ until the phage were pelleted on the cell bottom and then, increasing the speed to 33,450 rpm, it was possible to examine the phage and DNA boundaries in a single centrifuge run and to see if components of intermediate sedimentation coefficient were present. Such an experiment is shown in Fig. 3, in which it is clearly seen that there is no material other than intact phage $\left(\mathrm{S}_{20, \mathrm{w}}=487\right)$ and free $\mathrm{DNA}\left(\mathrm{S}_{20, \mathrm{w}}=29\right)$. Hence, intermediate states, if they exist, must be either very short-lived or unstable in $1 \underline{\mathrm{M}} \mathrm{NaClO}_{4}$.

The kinetics of release were then examined by incubating the phage in high concentrations of $\mathrm{NaClO}_{4}$ for various times and stopping the reaction by diluting to 1.0 molar. The percentage of DNA released was measured with the ultracentrifuge as described in the Methods section; Fig. 4 shows the surprising results of the measurements. At $2.3 \mathrm{M} \mathrm{NaC10}$, $50 \%$ of the phage release their DNA in 1 min; the remaining phage (which are nonviable) are stable for $20 \mathrm{~min}$, after which they gradually release their DNA over a 16-min interval. Although the T7M stock was isolated from a single plaque several years ago, it was possible that during serial transfer the population had become mixed. Hence, a single plaque was reisolated and a new sample of purified phage was prepared. The kinetics of release in $2.3 \mathrm{M} \mathrm{NaClO}_{4}$ were then remeasured and, as also shown in Fig. 4, the curves are indistinguishable. These measurements have been repeated with a second single plaque isolate and with a second preparation from the original stock; each time the same curve for the kinetics of release in $2.3 \underline{M}$ has resulted.

It seems clear that a $T 7$ phage population consists of two fractions existing in approximately equal numbers and showing rather different sensitivities to $\mathrm{NaClO}_{4}$. One fraction releases DNA very rapidly in a way that is relatively independent of perchlorate concentration; the other is highly concentration-dependent. In an effort to see if the DNA released at each stage was the same, a sample was treated with $2.3 \mathrm{M} \mathrm{NaClO}$ for $15 \mathrm{~min}$, diluted, and the phage, which had not released DNA, was collected by centrifugation for $90 \mathrm{~min}$ at $22,000 \mathrm{rpm}$ 


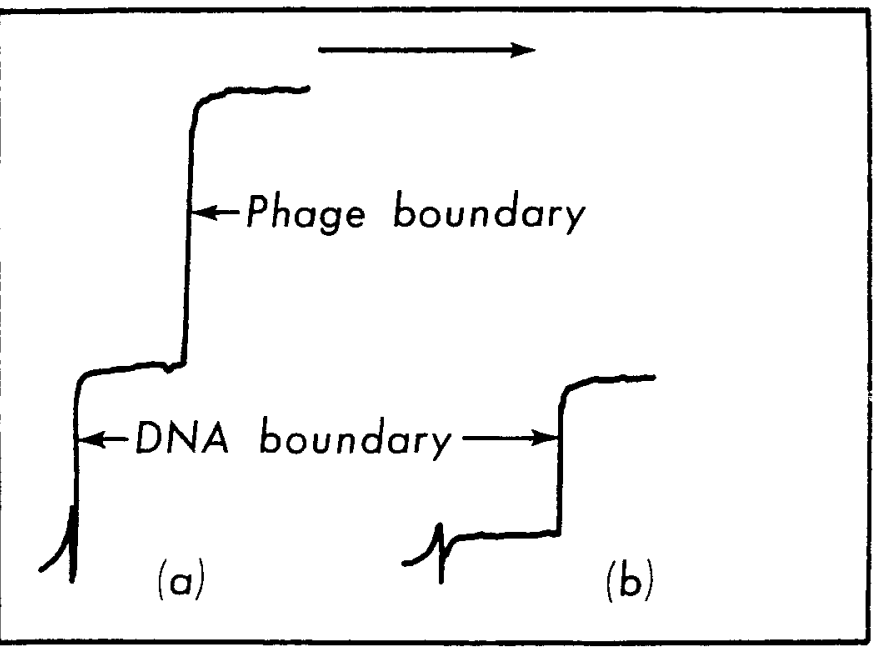

Figure 3. Photometric trace of an ult raviolet absorption photograph of a sedimentation distribution diagram of $T 7$ phage treated for $15 \mathrm{sec}$ with $2.3 \mathrm{M} \mathrm{NaClO}_{4}$ and diluted. The distribution after sedimentation for $10 \mathrm{~min}$ at $12,590 \mathrm{rpm}$ is shown in (a). At this time, the only material trailing behind the phage boundary is sharp DNA boundary which has not yet begun to sediment. (b) is the situation $12 \mathrm{~min}$ after reaching 33,450 rpm. The phage has sedimented to the cell bottom and the sharp DNA boundary is evident.

MUB-7518

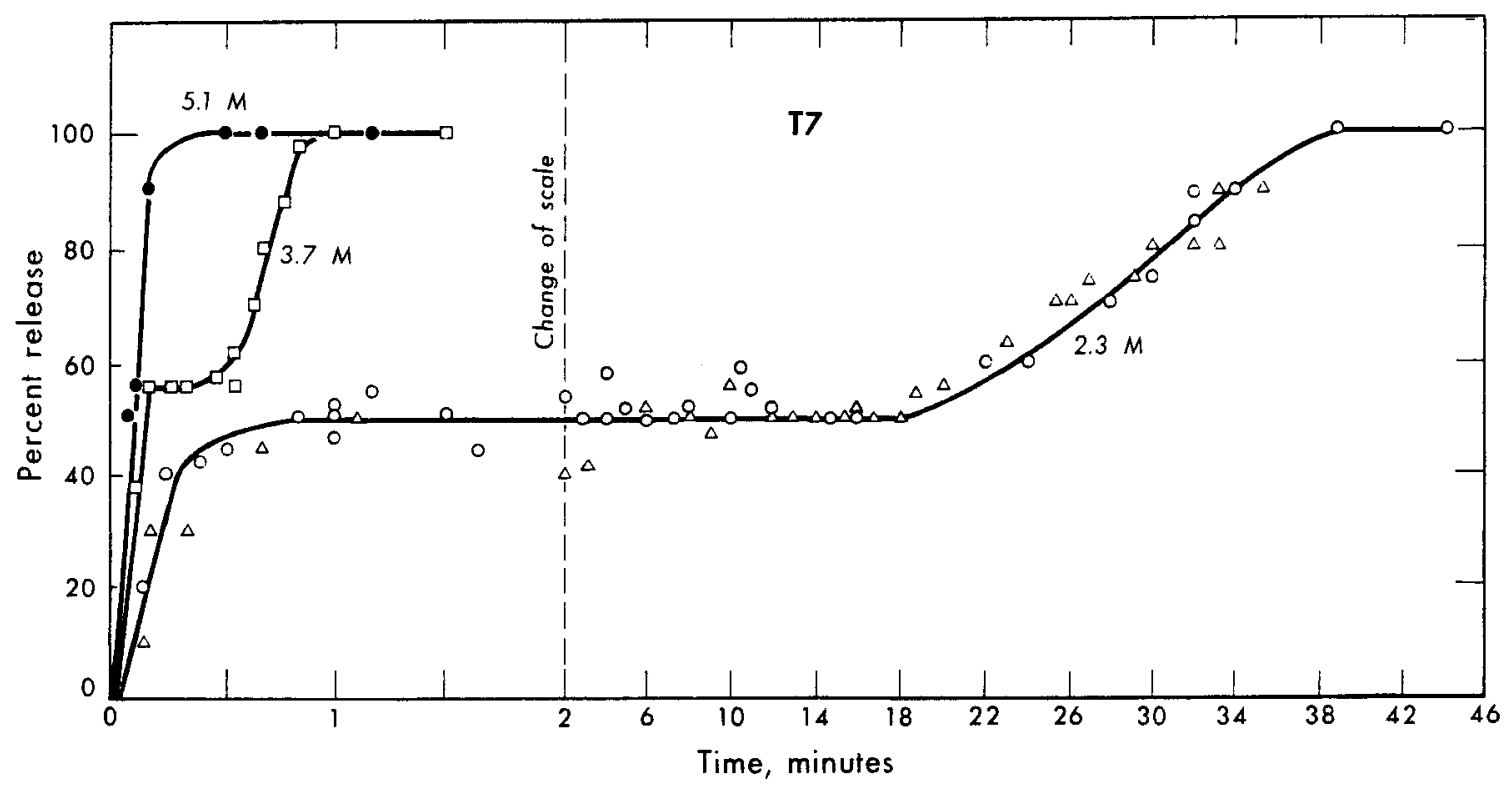

Figure 4. Kinetics of release of DNA from phage $\mathrm{T} 7$ in various concentrations of $\mathrm{NaClO}_{4}$. The ordinate is the percentage of the phages which have released DNA. For $2.3 \mathrm{M} \mathrm{NaClO}_{4}$ two sets of points are given. One $(\Delta)$ is for the same phage preparation used for the other concentrations; the other set $(O)$ is for a phage preparation made from a single plaque. 
in the SW39 rotor of the Spinco Model L Ultracentrifuge. The DNA was then released from these phages both by heating and by 25 minutes' incubation in $2.3 \mathrm{M} \mathrm{NaClO}_{4^{\circ}}$ Each DNA sample was also denatured by alkali. The centrifuge boundaries for both native and denatured DNA from the first-released fraction were indistinguishable from those of the second-release fraction.

STUDIES WITH E. coli PHAGE T $40^{\mathrm{r}}$ The T $40^{\mathrm{r}}$ mutant, resistant to osmotic shock, was chosen to eliminate the possibility that DNA was released simply by osmotic rupture of the phage during dilution. The possibility was actually not considered likely since, with T7, a DNA boundary was found in the centrifuge when the $2.3 \mathrm{M}$ solution was examined directly without dilution. Studies with $T 40^{\mathrm{r}}$ eliminated this possibility since all attempts to release $\mathrm{T} 40^{\mathrm{r}}$ DNA by rapid dilution from $5 \underline{\mathrm{M}} \mathrm{NaCl}$ failed, whereas with slow dilution (dialysis) from $5 \underline{\mathrm{M}}$ $\mathrm{NaClO}_{4}$, following a 5 -min incubation, all of the DNA was released.

The experiments carried out with $T 7$ were repeated for $T 4$. Using $5.1 \mathrm{M} \mathrm{NaClO}_{4}$ for 5 min and comparing to DNA released by pH 11, as previously described, it was found that perchlorate-released DNA shows the usual sedimentation coefficient and boundary shape. With care to avoid hydrodynamic shear, a single sharp boundary resulted. In contrast with $\mathrm{T}$, sedimentation at $8,766 \mathrm{rpm}$ followed by 15,220 rpm demonstrated a third boundary with a sedimentation coefficient other than that of whole phage or free DNA. The sedimentation coefficient of this boundary was 720 compared to 1,025 for the original phage and 53 for the DNA. This is the sedimentation coefficient of the slow, short-head form of $\mathrm{T} 4$ described by Cummings and Kozloff (7). Hence, the perchlorate probably triggers the transition from the long- to the short-head form. It has been suggested that the short-head form occurs before or during injection of the DNA. The possible validity of this conclusion in the present situation will be briefly considered later. The released DNA was unaffected by heating for $5 \mathrm{~min}$ at $70^{\circ} \mathrm{C}$ in $1 \mathrm{M} \mathrm{Na}{ }^{+}$. There was, however, an effect of heating on perchlorate-treated phages that had not yet released DNA. Normally, $T 40^{\mathrm{r}}$ will not release DNA when heated as does the $M \mathrm{mu}-$ tant of $T 7$. However, after treatment with $\mathrm{NaClO}_{4}$ concentrations resulting in partial release, i. e., heating of the phage at $70^{\circ} \mathrm{C}$ results in further release of DNA.

The kinetics of release of $T 4$ are qualitatively similar to those for $T 7$, as shown in Fig. 5. Release is rapid in $5.1 \mathrm{M} \mathrm{NaClO}_{4}$ and occurs in a single stage, yet in $4.3 \mathrm{molar}$, bimodal kinetics are again evident with 50\% of the phage releasing the DNA rapidiy and the remainder releasing later and at a lower rate. In contrast with $\mathrm{T} 7$ there is virtually no release below $4 \mathrm{M}$.

ST UDIES WITH Pseudomonas aeroginosa PHAGE B3 The experiments carried out for $T 7$ were repeated with B3 in order to examine the features of the perchlorate reaction with phages from hosts other than E. coli. Figure 6 shows the kinetics of release with various concentrations of $\mathrm{NaClO}_{4}$. The results are essentially like those for the other phages except for one important point. In $2.4 \mathrm{M} \mathrm{NaClO}_{4}$ it is found that the DNA often sediments with two boundaries having $S_{20, w}=29$ and 42 . The usual $S_{20, w}$ for phenol extracted B3 DNA is 29. The fast boundary is the first to appear and gradually is replaced by the normal $\mathrm{S}_{20, \mathrm{w}}=29$ 


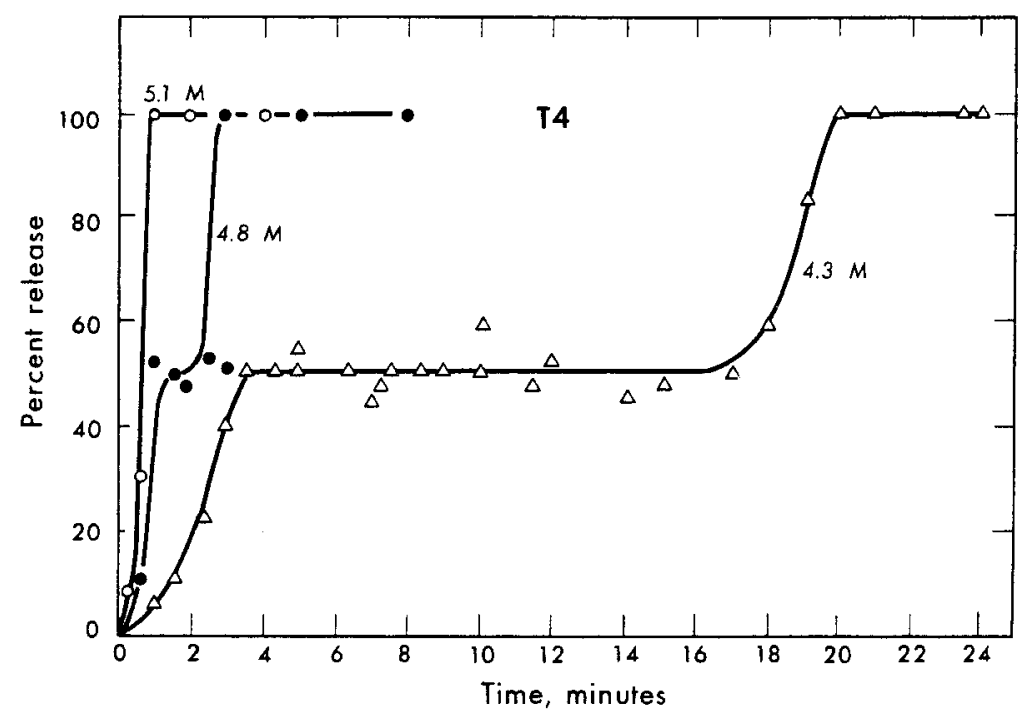

Figure 5. Kinetics of release of DNA from phage $T 40^{\circ}$ in various concentrations of $\mathrm{NaClO}_{4}$. The ordinate is the percentage of the phages that have released DNA.

$M U B-7520$

Figure 6. Kinetics of release of DNA from phage $B 3$ in various concentrations of $\mathrm{NaClO}_{4}$. The ordinate is the percentage of the phages that have released DNA.

MUB-7521

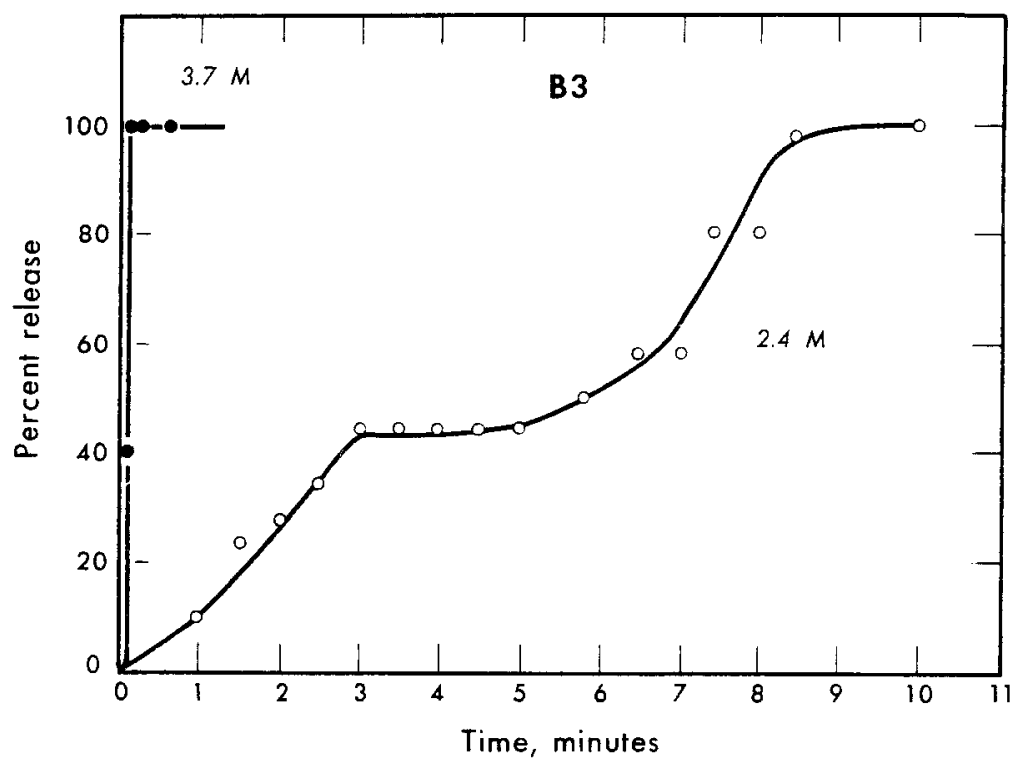


boundary (Fig. 7). After $10 \mathrm{~min}$ in $2.4 \mathrm{M} \mathrm{NaClO} 4$ all the DNA is in the normal form. In $5.1 \mathrm{M} \mathrm{NaClO} 4$ the fast form never appears. The fast form can be converted to the slow in several ways: I. overnight incubation in $1 \mathrm{M} \mathrm{NaClO}_{4}$ at $25^{\circ} \mathrm{C}$, II. incubation at pH 10 for $5 \mathrm{~min}$ at $25^{\circ} \mathrm{C}$, III. heating for $3 \mathrm{~min}$ at $67^{\circ} \mathrm{C}$ in $0.2 \mathrm{M} \mathrm{NaClO}, \mathrm{pH} 7.8$ (Fig. 8), and IV. hydrodynamic shear forces with shears too low to break the normal DNA molecule. It was concluded from these data that either the fast form is a more compact molecule than the normal, unlikely because of the conversion by shear, or that there is something loosely attached to the end of the DNA molecule. In an effort to understand this phenomenon, electron micrographs of the fast and slow forms were taken by Dr. Albrecht K. Kleinschmidt. Normal DNA (heator alkali-released) appeared as typical DNA filaments with a length of approximately $11-12 \mu$ (there were insufficient measurements to determine the length more accurately). However, the fast form appeared as a DNA filament with the phage ghost attached to one end, Fig. 9. The length of the attached filament was again ca. 11-12 . It was then observed that if the fast form was converted to the slow by heat or further incubation, the phage ghost was detached from the DNA although still visible in the micrographs. If DNA is released in $5.1 \mathrm{M} \mathrm{NaClO}_{4}$, normal filaments are seen along with unattached badly disrupted phage ghosts. These experiments (as well as those with $\mathrm{T} 40^{\mathrm{r}}$ ), which will be discussed in detail later, suggest that $\mathrm{NaClO}_{4}$ triggers the injection mechanism and causes the phage to inject the DNA through its tail. More data, bearing on this idea, will be given in the next section.

STUDIES WITH Bacillus subtilis PHAGE SP-50 Phage SP-50 is extremely sensitive to $\mathrm{NaClO}_{4}$. Concentrations as low as $0.1 \mathrm{M}$ inactivate the phage (Trautner, personal communication). Incubation for $10 \mathrm{sec}$ in $1 \underline{\mathrm{M}}$ is sufficient for DNA release. The boundary obtained by incubation in $5 \underline{\mathrm{M} \mathrm{NaClO}_{4}}$ is a normal boundary having $\mathrm{S}_{20, \mathrm{w}}=52(20 \mu \mathrm{g} / \mathrm{ml}, 27,690 \mathrm{rpm})$. However, when the DNA is released in $2 \mathrm{M} \mathrm{NaClO}$, the boundary is exceptional and consists of four principal regions: (1) The slowest material, which is a sharp boundary with $\mathrm{S}_{20, \mathrm{w}}=52$; (2) a fairly sharp boundary with $\mathrm{S}=75-80$, which is presumably analogous to the fast boundary of phage B3; (3) a very diffuse boundary where sedimentation coefficients range continuously up to (4) the boundary for intact phage. Electron micrographs of such a mixture were also taken by Kleinschmidt and Trautner (to be published), and it was found that not only are there phage ghosts attached to the DNA, but in many cases the DNA was not totally injected. Evidence for this latter point was that the attached filament was much shorter than normal whole molecules. Very few free short filaments were found so that the short attached filaments are probably not the resuit of breakage of the part that was initially injected. It is assumed that these short filaments represent partially injected molecules and comprise the third diffuse boundary described above. Further work on SP-50 is in progress (Trautner, personal communication).

ISOLATION OF DNA IN THE PRESENCE OF DNAase It should be remembered that this study was begun to determine whether $\mathrm{NaClO}_{4}$ could be used to isolate phage DNA in the presence of DNAase. To test this, purified phage $\mathrm{T} 7$ at $O \mathrm{D}_{260}=10$ was incubated at $25^{\circ} \mathrm{C}$ for $10 \mathrm{~min}$ in $0.01 \underline{\mathrm{M}} \mathrm{PO}_{4}, \mathrm{pH} 7.8$, and $10^{-4} \mathrm{M} \mathrm{MgCl}{ }_{2}$ containing $10 \mu \mathrm{g} / \mathrm{ml}$ pancreatic DNAase. This was divided,into four parts and the DNA was released respectively by (a) heating for 5 min at $70^{\circ} \mathrm{C}$, (b) $10 \mathrm{~min}$ at $25^{\circ} \mathrm{C}$ in $\mathrm{pH} 11.5$ phosphate, (c) shaking with phenol, and 


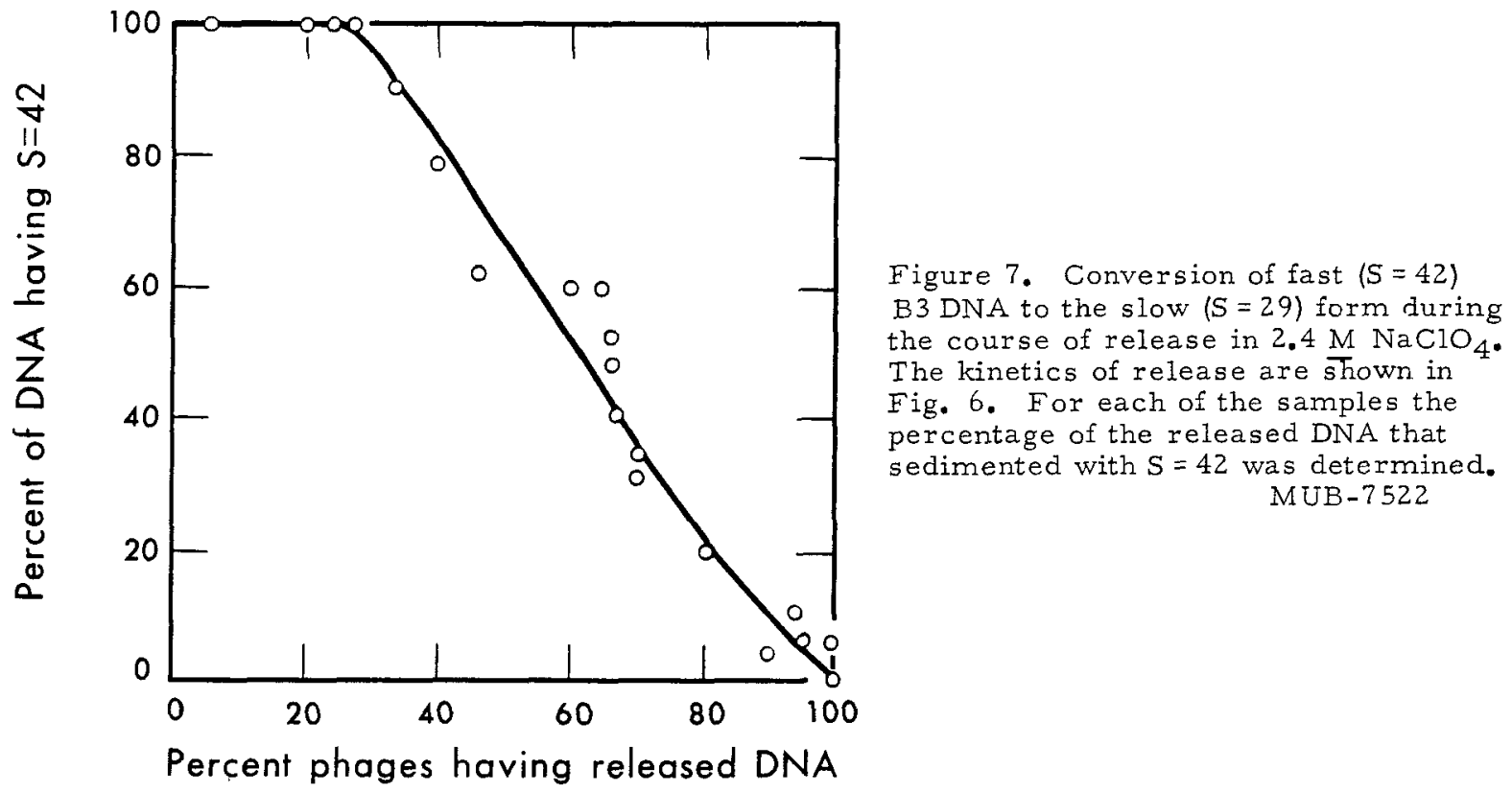

Figure 8. Conversion of fast ( $=42)$ B3 DNA to the slow $(S=29)$ form by incubation for $3 \mathrm{~min}$ at various temperatures. DNA was released from $\mathrm{B} 3 \mathrm{by}$ incubation for $2 \mathrm{~min}$ in $2.4 \mathrm{M}$ $\mathrm{NaClO}_{4}$. This was diluted to $0.2 \bar{M}$ and samples were heated for $3 \mathrm{~min}$ in a water bath at the indicated temperatures. MUB-7523

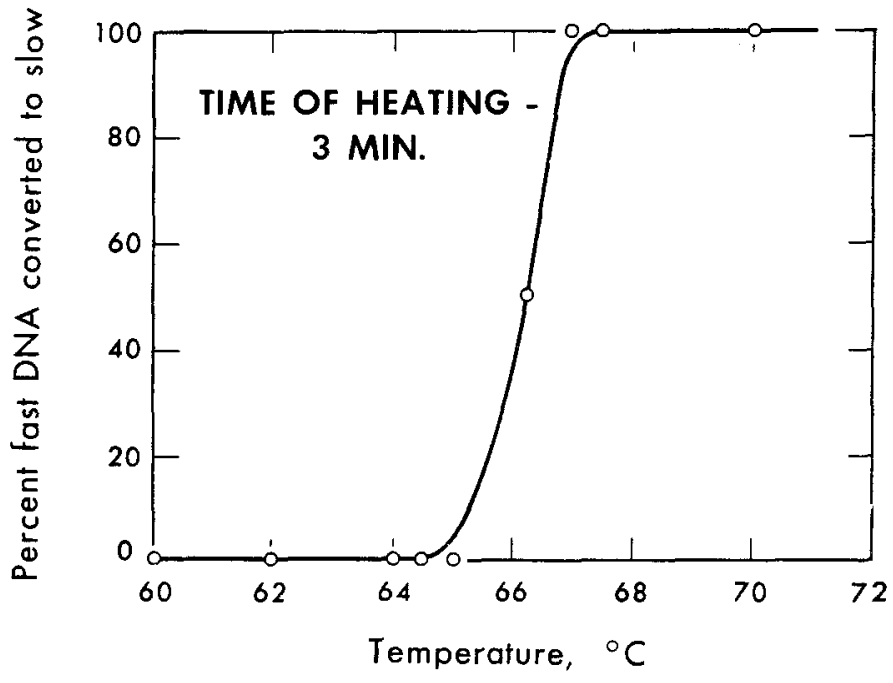




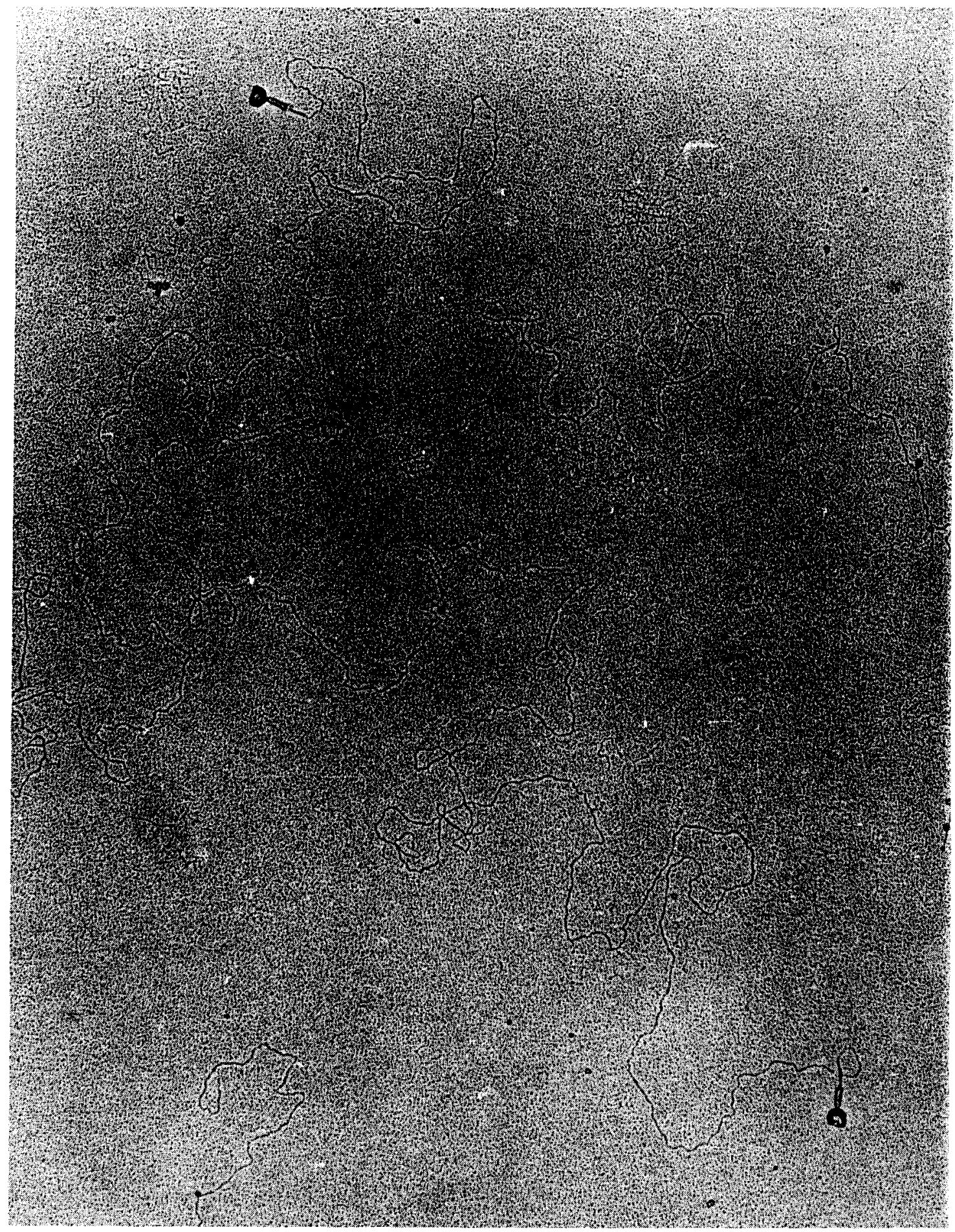

Figure 9. Electron micrographs of phage $\mathrm{B} 3$ treated with $2.4 \mathrm{M} \mathrm{NaClO}$ for $5 \mathrm{~min}$. The phage ghost is attached to the extruded DNA molecule. The block bar represents 1н. Micrograph by Dr. Albrecht K. Kleinschmidt. 
(d) $5 \mathrm{M} \mathrm{NaClO}_{4}$ for $3 \mathrm{~min}$. After release, $\mathrm{NaCl}$ was added to each to adjust the ionic strength to approximately 0.3. Then, each was incubated for $10 \mathrm{~min}$ at $37^{\circ} \mathrm{C}$, the DNA was alkalidenatured and single strand breaks were assayed by the technique of Davison, Freifelder and Holloway (4). In cases (a) and (b) no intact single strands were found; in (c) the percentage of intact strands was ca. 5-10\%; in (d) the percentage of broken strands was the usual value of $50 \%$ found for undamaged phage. Whether perchlorate protects by inactivation of the enzyme or simply by binding $\mathrm{Mg}$ is unclear, although carrying out the entire reaction in the presence of $0.002 \underline{M}$ versene did not protect the DNA.

\section{DISCUSSION}

DNA can be released from bacteriophages by incubation in $\mathrm{NaClO}_{4}$. The precise conditions of concentration and time must be determined for each particular phage in order to get total release. This technique is valuable because 1) the reagents used do not have appreciable absorption at $260 \mathrm{m \mu}$, 2) the procedure is rapid, 3) arbitrarily small volumes can be used, 4) DNAase is rendered ineffective, and 5) the DNA is presumably undamaged.

From the observations that the tail of $\mathrm{T} 4$ becomes contracted and the DNA is attached to the phage ghost at the tail for the cases of B3 and SP-50, it can be concluded that the mechanism of release at low concentrations is to trigger the injection apparatus so that the phage injects its DNA through the tail into the medium. In this respect, it is like the CdCN-Iysine reagent of Kozloff, Lute and Henderson (8). This probably accounts for the very rapid release of DNA by low concentrations of $\mathrm{NaClO}_{4^{\circ}}$. With longer incubation, the percentage of released DNA increases slowly. The appearance of badly damaged ghosts in electron micrographs of such preparation suggests that this slow release is a result of total disruption of the phage head. Hence, one must assume, at least for $T 4$ and $T 7$, that a phage population (derived originally from a single plaque) is heterogeneous in that half will inject their DNA under the influence of $\mathrm{NaClO}_{4}$ while the remaining half will not. The cause of this heterogeneity is unclear. One might speculate that injection has a preferential direction and that the DNA in the $50 \%$ noninjectors is in some way improperly oriented.

The result with $B 3$ and SP-50, i. e., the attachment of the DNA to the tail of the phage ghost indicates that there must be a DNA-protein bond which is broken by $\mathrm{NaClO}_{4}, \mathrm{pH} 10$, phenol and temperature. The very sharp thermal transition (Fig. 8) suggests that this bond must be rather exceptional. It should be pointed out that one cannot conclude that in other phages, e.g., T4 and T7 where the fast form is not found, this bond is not present, because it may be broken by the minimum exposure to $\mathrm{NaClO}_{4}$ required for release. The significance of this bond is unclear; it could be involved either with normal injection into bacteria or with packaging of DNA into phage particles. Perhaps the 50\% noninjectors referred to above are those phage for which the "wrong" end of the DNA is attached to this bond.

A final remark can be made about a special use of the perchlorate reagent. In many phage experiments, it is desirable to eliminate the unadsorbed phages some time after infection. This can be done by addition of very low $(0.1$ to $0.3 \mathrm{M})$ concentration of $\mathrm{NaClO}_{4}$ to damage the adsorption apparatus. This can then be diluted out so that progeny phage are unharmed. 


\section{SUMMARY}

Bacteriophages treated with $\mathrm{NaClO}_{4}$ release their DNA. The rate of release depends upon $\mathrm{NaClO}_{4}$ concentration and varies with phage type. There are apparently two mechanisms of release: (1) at low perchlorate concentrations the DNA is injected through the phage tail, (2) at high concentrations the phage head is disrupted. For several phages examined only $50 \%$ of the particles will release by the injection mechanism. It has also been found that in some situations the DNA is injected but remains attached to the phage ghost by means of an unknown bond which is thermally labile and broken by high perchlorate concentrations. In all cases the perchlorate-released DNA is indistinguishable from DNA released by more standard methods.

\section{ACKNOWLEDGMENTS}

I wish to thank Katherine Le Blanc, who prepared the purified phages and did the survival measurements; Professors Albrecht K. Kleinschmidt and Thomas A. Trautner for many helpful discussions, for unpublished results and the excellent electron micrograph of B3; and Martin Haas, to whom I am grateful for the electron micrographs of T4.

\section{REFERENCES}

1. Hamaguchi, K., and Geiduschek, E. P.; J. Am. Chem. Soc. 84: 1329, 1962.

2. Freifelder, D.; Biochem. Biophys. Res. Commun. 18: 141, 1965.

3. Davison, P. F.; Freifelder, D., and Holloway, B. W.; J. Mol. Biol. 8: 1, 1964.

4. Davison, P. F., and Freifelder, D.; J. Mol. Biol. 5: 643, 1962.

5. Brenner, S.; Streisinger, G.; Horne, R. W.; Champe, S. P.; Barnett, L.; Benzer, S., and Rees, M. W.; J. Mol. Biol. 1: 281, 1959.

6. Freifelder, D., and Davison, P. F.; Biophys. J. 3: 49, 1963.

7. Cummings, D. L., and Kozloff, L.; Biochim. Biophys. Acta 44: 445, 1960.

8. Kozloff, L.; Lute, M., and Henderson, K.; J. Biol. Chem. 228: 511, 1957.

Received November, 1965. 


\title{
Recovery of Yeast After Exposure to Densely lonizing Radiation
}

\author{
John T. Lyman and Robert H. Haynes
}

It has long been recognized that the metabolic stability of living cells depends upon a balance between biochemical breakdown and repair processes. Furthermore, at many levels of organization, homeostatic mechanisms produce responses to environmental stresses that tend to preserve the integrity of the organism. A number of recovery phenomena associated with radiation injury have recently been attributed to the enzymic repair of DNA structural defects. This might be regarded as an example of a "homeostatic" mechanism operating at the macromolecular level.

In view of the importance of recovery processes in radiobiology, it is essential to determine what types of radiation damage are susceptible of repair in various kinds of cells. In diploid yeast (Saccharomyces cerevisiae), viability is increased if, instead of being plated immediately after irradiation, the cells are incubated in a nonnutritive suspension for a few hours prior to being plated (1). A problem in terminology arises in the description of these observations. It is both tempting and convenient to use the word "recovery" to denote such an increase of viability. However, by this usage we do not wish to imply that the phenomenon is necessarily based upon the repair of macromolecular defects (e.g., as in enzymic photoreactivation or dark reactivation in E. coli); that is, we cannot rule out other mechanisms such as the prevention of expression or the "bypass" of the damage.

There is considerable evidence that densely ionizing particles produce a greater proportion of irreversible damage than $X$ rays $(2,3)$. Thus, it is of some interest to know whether or not recovery can occur in yeast irradiated with heavy ions, since it is not implausible that irreparable macromolecular damage might be produced within the columns of dense ionization formed along the particle tracks. However, we found that after heavy-ion irradiation, recovery occurs to the same extent as is observed after Xirradiation. Also, it would appear that recovery is additive to the increased viability arising from glycerol protection and is not caused by sporulation during the postirradiation incubation period (4).

\section{MATERIALS AND METHODS}

The physical and biological techniques used in these experiments have been described elsewhere, and only a brief summary of the most important points is given here (4). The Xray source was a Norelco OEG-60 beryllium-window tube operated at $50 \mathrm{kV}$ and $25 \mathrm{~mA}$ without any external filtration. Under these conditions the dose rate to the cells was $15 \mathrm{krads}$ per minute. The source of heavy-ion radiation was the Berkeley Hilac. Stripped nuclei of helium, carbon, and neon with energies of approximately $9 \mathrm{MeV}$ per atomic mass unit were used. 
In each experiment, the radiation doses were measured with a thin, transmission-type ionization chamber placed in the beam about $1 \mathrm{~mm}$ before the sample of cells. The dose rates employed varied between 6 and $15 \mathrm{krads}$ per minute in various runs.

The mass stopping power $\left(\frac{1}{\rho} \frac{d E}{d x}\right)$ of tissue for the ions is given in Table 1 . This simple measure of the collisional energy losses of heavy charged particles is sufficient for the purpose of these studies. We restrict the use of the quantity LET (linear energy transfer) to cases in which it is necessary to distinguish between the energy losses along the tracks of the particles and the losses associated with those $\delta$ electrons scattered beyond the primary track "core" $(5,6)$. Thus, the calculation of $\frac{1}{\rho} \frac{\mathrm{d} E}{\mathrm{dx}}$ includes all the $\mathrm{o}$-electron losses even though part of this energy is deposited some distance from the primary tracks.

Table 1. Energy and stopping power for heavy ions used for irradiation of yeast strain X841.

\begin{tabular}{lcc}
\hline Radiation & $\begin{array}{c}\text { Energy } \\
(\mathrm{MeV} / \mathrm{amu})\end{array}$ & $\begin{array}{c}\text { Stopping power } \\
\text { of tissue } \\
\left(\mathrm{MeV}-\mathrm{cm}^{2} / \mathrm{g}\right)\end{array}$ \\
\hline${ }^{4} \mathrm{He}$ & -9 & 180 \\
${ }^{12} \mathrm{C}$ & 9.1 & 1765 \\
$20_{\mathrm{Ne}}$ & 8.2 & 4920 \\
\hline
\end{tabular}

Since the range of the particles is much greater than the thickness of the monolayer of irradiated cells, the maximum energy loss (at Bragg peak) occurs after the particles have passed through the cells. Thus, the energy loss in the cells for the $40-\mathrm{MeV}$ helium ions is much lower than that commonly associated with $\alpha$ particles, and in fact is close to that of $50-\mathrm{kV} X$ rays (7). The $\mathrm{RBE}$ (relative to $\mathrm{X}$ rays) for the inactivation of yeast goes through a maximum as the energy loss of the ions increases. The carbon ions, for which the energy loss is similar to that for the $\alpha$-particle Bragg peak, are associated with the maximum RBE; the helium and neon ions fall on either side of this maximum $(8,9)$.

Subcultures of the following strains of the yeast Saccharomyces cerevisiae were used: X841, a diploid; X841p, a diploid petite; and XJ4, a diploid homozygous mating type ( $\alpha \alpha)$. All were grown in liquid YEPD medium ( $1 \%$ yeast extract, $2 \%$ peptone, $2 \%$ dextrose) for $48 \mathrm{hr}$ (96 hr for the petite strain) after which time the population is in stationary phase. The cells were harvested, washed three times and resuspended in double-distilled water. Microscopic examination of the suspension usually revealed less than $3 \%$ budding cells, no asci and no clumps.

Aliquots $(50 \lambda)$ of the suspension were placed on sterile 13-mm-diameter Millipore filters (type HA). The suspending liquid was quickly absorbed by an absorbent pad placed under the filter, leaving the cells in a monolayer on the surface. The pad was then saturated with double-distilled water. Because of the high porosity of the filter, although the cells remained wet, the water layer over the cells was not thick enough to reduce appreciably the beam energy. The cells were exposed to the air during irradiation, after which they were 
resuspended in double-distilled water. Samples were then plated on YEPD agar (immediate plating), and the remainder of the suspension was incubated for times up to $48 \mathrm{hr}$ at $30^{\circ} \mathrm{C}$. Further samples were plated at various times after irradiation in order to observe recovery (delayed plating). All plates were incubated for five days at $30^{\circ} \mathrm{C}$.

\section{RESULTS AND DISCUSSION}

RECOVERY IS INDEPENDENT OF LET Immediate and delayed plating dose-survival curves for the diploid yeast strain X841 irradiated with $50-\mathrm{kV} \mathrm{X}$ rays and ${ }^{4} \mathrm{He},{ }^{12} \mathrm{C}$, and $20 \mathrm{Ne}$ ions are shown in Figs. 1-3. It is clear from these results that recovery upon delayed plating occurs even after irradiation with the very densely ionizing ${ }^{20} \mathrm{Ne}$ particles $\left(\frac{1}{\rho} \frac{\mathrm{dE}}{\mathrm{dx}}=4920 \mathrm{MeV}-\right.$ $\mathrm{cm}^{2} / \mathrm{g}$ ). Furthermore, the magnitude of this recovery (measured as a dose-modifying factor: $\mathrm{DMF} \approx 2$ ) for all ions used is essentially the same as that observed for $\mathrm{X}$ rays. A similar recovery after irradiation with ${ }^{239} \mathrm{Pu} \alpha$ particles has been reported in Saccharomyces vini (10).

The shoulder on the survival curves does not decrease with increasing $\mathrm{dE} / \mathrm{dx}$, as has been observed in Aspergillus spores (11), Artemia eggs (12) and mammalian cells (3, 13). Clearly, yeast differs in this respect from other organisms of wide phylogenetic distribution. The reasons for this are by no means clear. However, it has been suggested that shoulders might be due to the saturation of or the inhibition of intracellular repair processes with increasing radiation dose $(14,15)$; recovery after ${ }^{12} \mathrm{C}$ and ${ }^{20} \mathrm{Ne}$ irradiation is at least consistent with the persistence of shoulders on the curves (9). Preirradiation treatment of diploid yeast with heat or ethyl methane sulfonate serves simultaneously to reduce the shoulder and to inhibit recovery (16). Thus, it is possible that recovery in yeast is related to and is perhaps an enhancement of a shoulder-producing repair process.

It has been shown that recovery after $X$ rays is unaffected by the presence or absence of oxygen during irradiation (1). A second line of reasoning may be invoked to relate this fact with recovery following denselyionizing radiation. The oxygen-dependent component of radiation damage has been attributed to the reaction of radicals produced by "small" ionization clusters, whereas larger clusters are believed to give rise to the oxygen-independent component $(17,18)$. If this idea is correct, then it follows that the recovery process is equally effective in dealing with lethal damage produced by both "small" and "large" ionization clusters. Therefore, the predominance of "large" clusters associated with the heavy-ion tracks might not be expected to reduce recovery.

Recovery appears to be completely additive to glycerol protection at the time of ir radiation. The protection afforded by $6 \underline{\mathrm{M}}$ glycerol is equivalent to a dose-modifying factor of about 2.6 and is independent of LET (8). Diploid yeast irradiated with either X rays or helium ions benefits from this protection, and in addition exhibit a full normal recovery DMF, also approximately 2.6, upon storage in distilled water. Thus, nearly a sevenfold reduction in the effective radiation dose may be achieved by a combination of glycerol protection and delayed plating. The general properties of recovery in yeast and the dark reactivation mechanisms in $\underline{E}$. coli $B / r$ are similar in that both appear to be enzymic, energy-requiring processes capable of repairing some fraction of the lethal damage produced by $\mathrm{X}$ rays, 


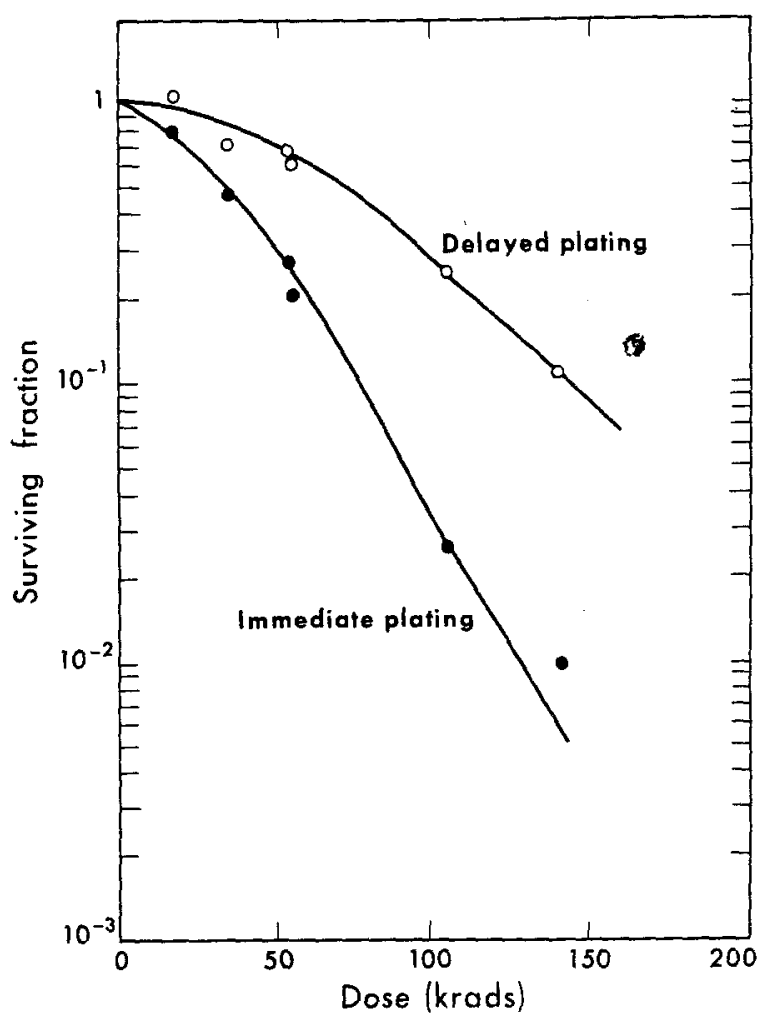

Figure 1. Immediate and delayed $(48 \mathrm{hr})$ platings of $\mathrm{X} 841$ following $50-\mathrm{kV} \mathrm{X}$ ir radiation. $M U-35519$

2537 - $\AA$ ultraviolet light, or nitrogen mustard, but none of the damage produced by heat or acridine-sensitized photodynamic action (1,15). However, a significant difference between the two processes is that whereas recovery in yeast is independent of $\mathrm{dE} / \mathrm{dx}$ for ionizing radiation, this is not the case in E. coli. Preliminary studies indicate that the sensitivity ratio of the reactivating and nonreactivating coli strains $B / r$ and $B_{s-1}$ declines from a value of 3.0 for $\mathrm{X}$ rays to 1.4 for ${ }^{20} \mathrm{Ne}$ ions and $210 \overline{\mathrm{Po}}$ a particles $(19,20)$. Thus, it would appear that damage caused by densely ionizing radiation cannot be repaired in $\mathrm{E}$. coli $\mathrm{B} / \mathrm{r}$. These contrasting results could be explained if recovery in yeast were based upon a "bypass" of the damage such as might occur during somatic recombination or sporulation.

RECOVERY UNRELATED TO SPORULATION Since haploid and petite diploid strains are incapable of either recovery or sporulation, a common pathway might be shared by the two processes (1,21). Furthermore, we have observed asci in stored suspensions of diploid cells. Despite these facts, the following observations tend to invalidate the suggestion that recovery is due to sporulation during the storage period:

1. Sporulation does not occur under anoxic conditions and is depressed at oxygen concentrations above $40 \%$ (22). Recovery is only moderately depressed under anoxic conditions and is enhanced in the presence of $100 \%$ oxygen (16). 


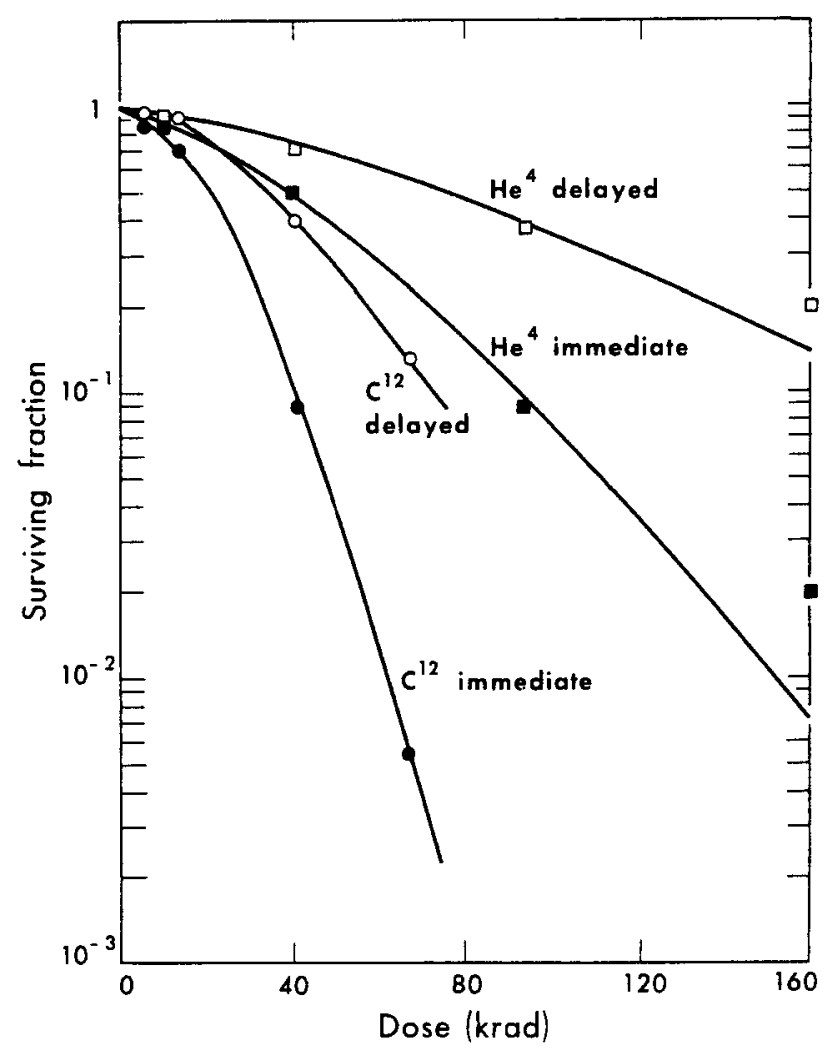

Figure 2. Immediate and delayed $(48 \mathrm{hr})$ platings of X841 following heavy-ion

$\left({ }^{12} \mathrm{C}\right.$ and $\left.{ }^{4} \mathrm{He}\right)$ irradiations, $\mathrm{MU}-35520$

Figure 3. Immediate and delayed (48 hr) platings of X841 following $20 \mathrm{Ne}$ irradiation. MU-35521

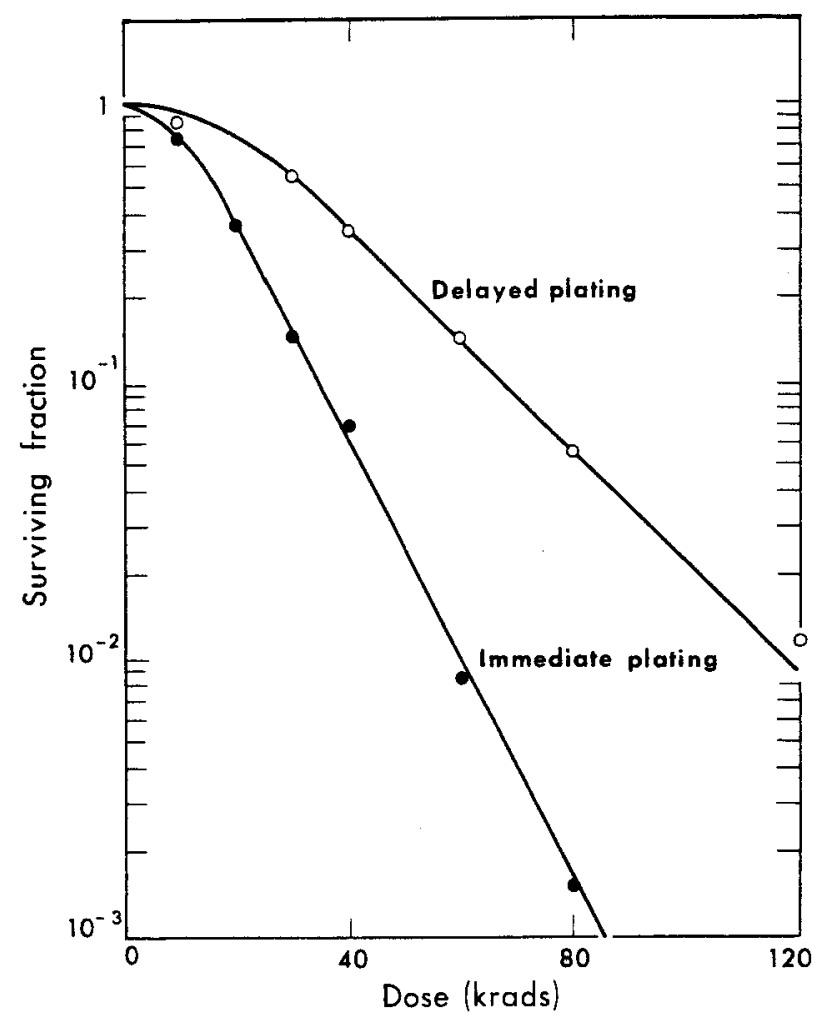


2. Dextrose concentrations between 0.3 and $1 \%$ in the storage medium tend to depress sporulation (23) whereas recovery is largely unaffected. The recovery of X-irradiated cells upon storage in dextrose-phosphate buffer $(0.5 \%$ dextrose) is shown in Fig. 4.

3. Sporulation depends upon cell concentration and is optimal at titers near $2 \times 10^{6} \mathrm{cells} / \mathrm{ml}(24)$. Recovery shows no such concentration dependence (4).

4. Sporulation is sharply inhibited at temperatures above $33^{\circ} \mathrm{C}(22)$, whereas recovery is still evident at $37^{\circ} \mathrm{C}(16)$.

5. Diploid strains that are homozygous at the "mating type" locus are incapable of sporulation (25). We tested the homozygous strain XJ4 ( $\alpha \alpha$ mating type) and found it was capable of recovery after $\mathrm{X}$ irradiation (Fig. 5).

These facts make it clear that recovery cannot be attributed simply to sporulation during the storage period, although they do not serve to rule out the possibility that the two processes have some enzymic steps in common.

\section{SUMMARY}

A great enhancement of viability is observed if nonnutritive suspensions of diploid yeast, which have been irradiated with $X$ rays or heavy ions $\left({ }^{4} \mathrm{He},{ }^{12} \mathrm{C},{ }^{2} 0 \mathrm{Ne}\right)$, are stored at $30^{\circ} \mathrm{C}$ in the dark for four or more hours prior to plating. Maximum recovery is usually observed after 24 to $48 \mathrm{hr}$; the survival curves obtained upon delayed plating are related to those for immediate plating by a constant dose-modifying factor. Several lines of evidence indicate that recovery is based upon enzymic postirradiation processes unrelated to the initial physicochemical reactions associated with absorption of the radiation. The magnitude of recovery is independent of such radiobiological modifiers as oxygen or glycerol, or track ion density. All these modifiers are thought to act by affecting the nature and distribution of the products of the initial radiochemical reactions. Thus, the recovery appears to be substantially independent of the precise chemical nature of the radiation-induced lesions. Very severe macromolecular damage is likely to be produced by the densely ionizing radiations. The ability of diploid yeast to recover after such irradiation suggests that a "bypass" rather than a direct repair mechanism is involved. Segregation of the damage by sporulation would appear to be, a priori, a suitable bypass mechanism, but this hypothesis is ruled out by the results cited in this paper.

\section{REFERENCES}

1. Patrick, M. H.; Haynes, R. H., and Uretz, R. B.; Radiation Res, 21: 144-163, 1964.

2. Barendsen, G. W., and Walter, H. M. D. ; Radiation Res. 21: 314-329, 1964.

3. Todd, P. W.; Ph. D. Thesis, University of California, Lawrence Radiation Laboratory Report UCRL-11614, 1964.

4. Lyman, J. T.; Ph. D. Thesis, University of California, Lawrence Radiation Laboratory Report UCRL-16030, 1965.

5. Burch, P. R. J., and Bird, P. M.; in Progress in Radiobiology, edited by J. S. Mitchell, B. E. Homes and C. L. Smith, Edinborough and London, Oliver and Boyd, 1956, pp. 161 174.

6. Burch, P. R. J.; Radiation Res. 6:289-301, 1957. 


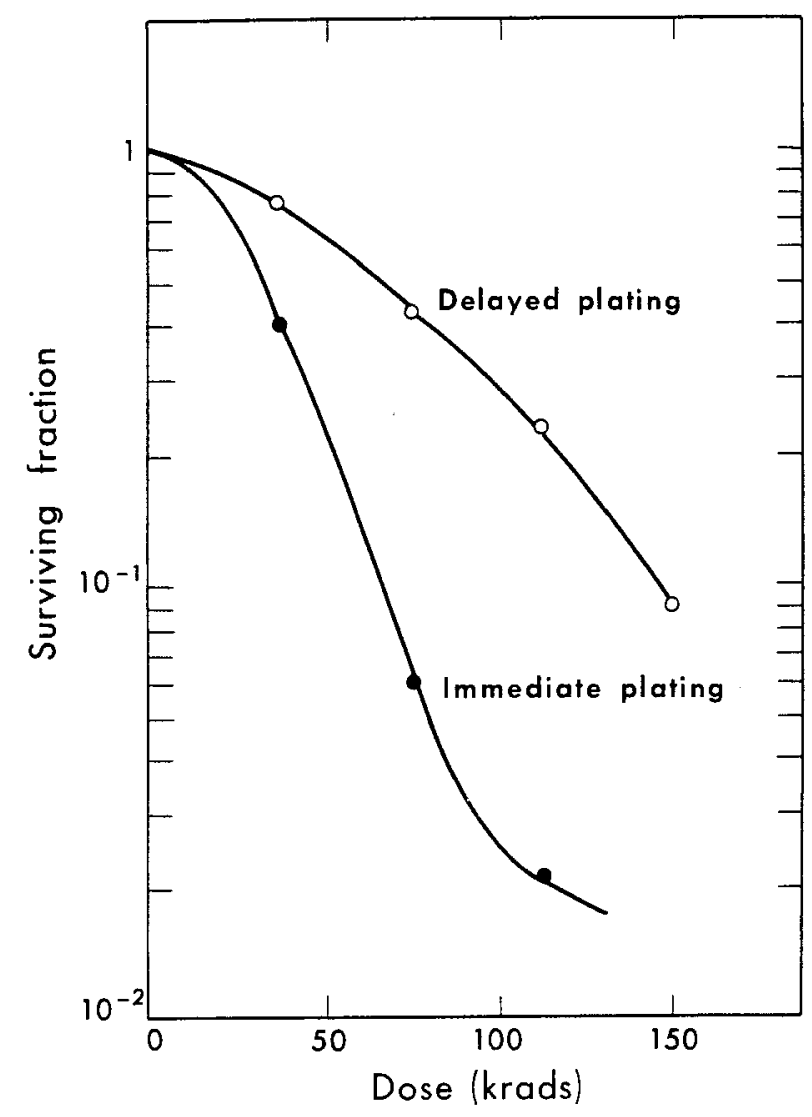

Figure 4. Immediate and delayed (24 hr) platings of $X 841$ following $50-\mathrm{kV} X$ rays. Cells were stored in dextrose-phosphate buffer $10.5 \%$ dextrose; $M / 15$ phosphate; pH 6.4).

MU-35524

Figure 5. Immediate and delayed (24 hr) platings of $\mathrm{XJ} 4$ following $50-\mathrm{kV} X$ rays. MU-35525

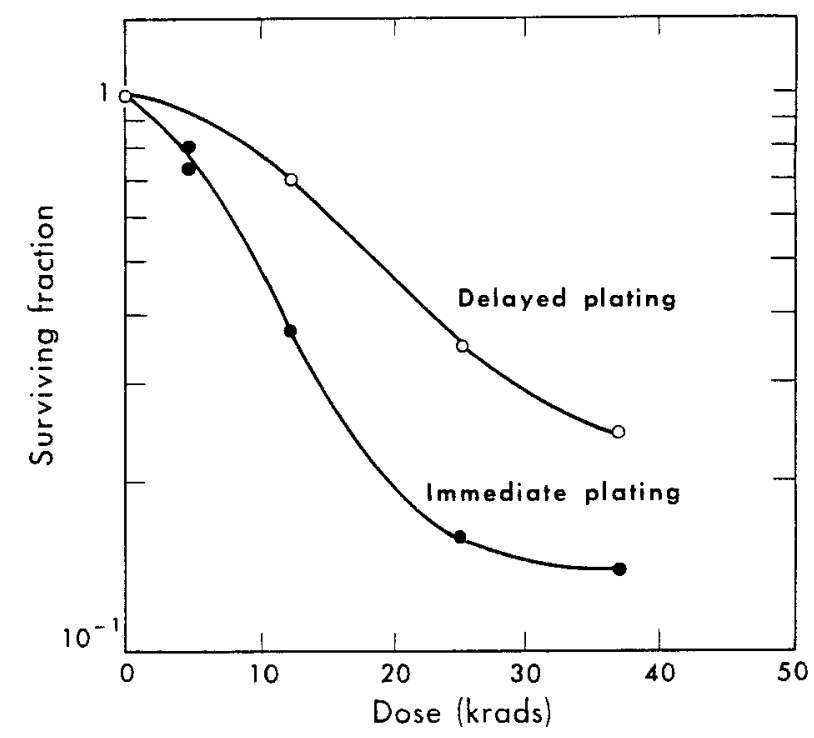


7. Haynes, R. H., and Dolphin, G. W.; Phys. Med. Biol. 4: 148-159, 1959.

8. Manney, T. R. ; Brustad, T., and Tobias, C. A.; Radiation Res. 18: 374-388, 1963.

9. Mortimer, R. H.; Brustad, T., and Cormack, D. V.; Influence of Iinear Energy Transfer and Oxygen Tension on the Effectiveness of Ionizing Radiations for Induction of Mutations and Lethality in Saccharomyces cerevisiae. Radiation Res.. in press.

10. Bilushi, V., and Korogodin, V. I. ; Dokl. Akad. Nauk SSSR 138: 1208-1211, 1961.

11. Zirkle, R. E.; Marchbank, D. F., and Kuck, K. D.; J. Cellular Comp. Physiol. 39, Suppl. 1: 75-85, 1952.

12. Easter, S. S., Jr., and Hutchinson, F.; Radiation Res. 15:333-340, 1961.

13. Barendsen, G. W.; Beusker, T. L. J.; Vergroesen, A. J., and Budke, L.; Radiation Res. 13: 841-849, 1960.

14. Haynes, R. H.; in Physical Processes in Radiation Biology, edited by L. Augenstein, R. Mason and B. Rosenberg, New York, Academic Press, 1964, 51-72.

15. Haynes, R. H.; Photochem. Photobiol. 3:429-450, 1964.

16. Patrick, M. H. , and Haynes, R. H. ; Radiation Res. 23:564-579, 1964.

17. Alper, T., and Howard-Flanders, P.; Nature 178: 978-979, 1956.

18. Howard-Flanders, P.; Advances in Biological and Medical Physics, Vol. 6, edited by C. A. Tobias and J. H. Lawrence, New York, Academic Press, 1958, pp. 553-603.

19. Haynes, R. H.; Patrick, M. H., and Baptist, J. E.; Radiation Res. 22: 194, 1964.

20. Inch, W. R.; (Donner Laboratory), personal communication, 1965.

21. Remezova, T. S., and Tret'Iakova, V. P.; Zh. Obshch. Biol. 22: 120-127, 1961.

22. Adams, A. M., and Miller, J. M.; Can. J. Botany 32:251-258, 1962.

23. Miller, J. J.; Can. J. Microbiol. 3: 81-90, 1957.

24. Miller, J. J.; Calvin, J., and Tremaine, J. H.; Can. J. Microbiol. 1: 560-573, 1955.

25. Mortimer, R. K.; invited discussion; Radiation Res. Suppl. 1: 394-402, 1959. 


\title{
Super-Suppressor Mutations in Saccharomyces cerevisiae
}

\author{
Richord A. Gilmore and Robert K. Mortimer
}

Suppressor mutations in the yeast Saccharomyces cerevisiae characterized by allele specificity and locus non-specificity were reported by Hawthorne and Mortimer (1). These "super-suppressors" were shown to affect approximately one-fourth of all the ultravioletinduced mutants in this yeast. Manney (2) has presented evidence that indicates these supersuppressors and the suppressible alleles in yeast are qualitatively similar to the amber suppressors and amber mutations in the bacteriophage - E. coli system. Considerable emphasis has been placed on the biochemistry of suppressors in E. coli, Benzer and Champe (3), Garen and Siddiqi (4), Brody and Yanofsky (5), Sarabhai et al. (6), Weigert and Garen (7), Brenner et al. (8) and Capecchi and Gussin (9), but relatively little work has been done to determine their numbers or distribution throughout the genome. Eggertsson and Adelberg (10) have examined suppressor mutations in E. coli and have found multiple-site suppressor loci that fell into four specificity groups. In addition, they reported the possibility of two suppressor loci that fall into a fifth specificity group which did not show multiple-site suppression. This paper describes genetic studies on super-suppressor loci in yeast.

\section{MATERIALS AND METHODS}

Two haploid strains of Saccharomyces cerevisiae were used. The genotypes of these

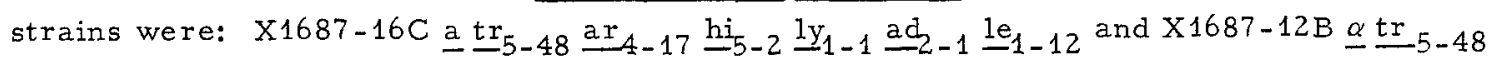
$\operatorname{ar}_{4-17} \underline{h i}_{-2} \underline{l y}_{1-1} a d_{2-1}$. With the exception of $\underline{l e}_{1-12}$, all the alleles are suppressible by the super-suppressor $\underline{S}_{d}$, Manney (2) and Mortimer, unpublished.

Media used were as follows:

YEPD (stock maintenance medium): 1\% Difco yeast extract, $2 \%$ Difco peptone, $2 \%$ dextrose, $2 \%$ agar.

COMP (synthetic complete) : $0.67 \%$ Difco Yeast Nitrogen Base without amino acids, $2 \%$ dextrose, $2 \%$ agar, plus the following, per liter, adenine, arginine, lysine, methionine, tryptophan, and uracil, $20 \mathrm{mg}$ each, leucine, $30 \mathrm{mg}$, and histidine, $10 \mathrm{mg}$.

Omission media: COMP lacking one of the amino acids or bases, e.g. -TR would be synthetic complete minus tryptophan, -AR would be synthetic complete minus arginine, etc.

GNA (presporulation medium): 5\% dextrose, $2.3 \%$ Bacto-nutrient agar, $1 \%$ yeast extract, $0.5 \%$ additional agar.

Raffinose acetate (sporulation medium): $0.022 \%$ raffinose, $0.3 \%$ sodium acetate. 
Genetic analyses to determine whether the suppressor mutations in the revertants were at the same or different loci were performed as follows. Suppressor-bearing $\underline{a}$ and $\underline{\alpha}$ strains were mated, the resultant diploid sporulated, and asci dissected by the method of Johnston and Mortimer (11). Spore cultures were streaked on YEPD plates and their phenotypes determined by replica plating onto omission media. If the two suppressors in the diploid were at the same locus, every spore would bear a suppressor. If the two suppressors in the diploid were at different loci, recombination would result in some spore colonies with no suppressor. The ability of the spore colonies to grow on the different omission media would indicate the presence of one or both of the suppressors since the suppressible alleles were homoallelic in all the diploids. Ultraviolet irradiation was performed with a dose of $5.37 \times 10^{4} \mathrm{ergs} / \mathrm{cm}^{2}$.

\section{RESULTS}

Ultraviolet-induced revertant colonies were isolated from each of the following omission media, $-T R,-A R,-H I,-L Y,-A D$. They were grown on YEPD and then replica plated onto the same five omission media. Any revertant colony that was able to grow on two or more of the five omission media was considered to contain a super-suppressor and was used in later tests. A total of 1,053 revertants were tested and 83 super-suppressors were obtained.

The suppressors can be grouped into eight phenotypic classes on the basis of phenotypic expression after seven days incubation (Table 1). Revertants from the two largest classes (I and III) were subjected to genetic analysis in order to determine the number of suppressor loci. Twenty revertants in class I were distributed over six genetic loci and ten revertants in class III were situated at four loci. Interclass crosses between the six loci of class I and the four loci of class III have shown all 10 loci to be distinct. Similar crosses of these 10 loci with known suppressors $\underline{S}_{b}$, Hawthorne and Mortimer (1), and $\underline{S}_{d}$ have established that a minimum of 12 distinct super-suppressor loci exist.

Approximately one-third of the 28 possible interclass combinations of suppressors have been tested on a limited basis. In these combinations, there has been no case of allelism detected. It thus appears that each phenotypic class represents a separate set of suppressor loci. This would make the minimum number of loci 18 if we assume at least one locus for each of the six other classes.

That the suppressible mutations are "nonsense" mutations, Benzer and Champe (3), Garen and Siddiqi (4) and Manney (2), has been reasonably well established, Weigert and Garen (7), and Brenner, Stretton and Kaplan (8). Concerning the involvement of s-RNA in suppression, Brenner, Stretton and Kaplan (8), and Capecchi and Gussin (9), several plausible models of suppressor action have been proposed. One of these models requires that a gene that normally makes a species of chargeables-RNA has a mutational event resulting in an alteration of the anticodon. The alteration then allows the species to translate the nonsense codon and insert its characteristic amino acid into the protein at that position. Since this is not a lethal mutation, this s-RNA must also be able to recognize its normal codon. Another 
Table 1.

\begin{tabular}{|c|c|c|c|c|c|c|c|c|}
\hline \multirow{2}{*}{$\begin{array}{l}\text { Phenotypic } \\
\text { class }\end{array}$} & \multicolumn{5}{|c|}{ Alleles suppressed } & \multirow{2}{*}{$\begin{array}{l}\text { Number of } \\
\text { revertants }\end{array}$} & \multirow{2}{*}{$\begin{array}{c}\text { Number of } \\
\text { revertants } \\
\text { tested } \\
\text { genetically }\end{array}$} & \multirow{2}{*}{$\begin{array}{c}\text { Number } \\
\text { of } \\
\text { loci }\end{array}$} \\
\hline & $\operatorname{tr}_{5-48}$ & $\operatorname{ar}_{4-17}$ & ${ }^{h i_{5-2}}$ & $1 y_{1-1}$ & $\mathrm{ad}_{2-1}$ & & & \\
\hline $\mathrm{I}$ & + & + & + & + & + & 41 & 20 & 6 \\
\hline II & + & + & + & + & - & 4 & - & \\
\hline III & + & + & + & - & - & 21 & 10 & 4 \\
\hline IV & + & + & - & + & - & 1 & - & \\
\hline $\mathrm{V}$ & + & + & - & - & - & 12 & - & \\
\hline VI & + & - & + & - & - & 1 & - & \\
\hline VII & + & - & - & + & - & 2 & - & \\
\hline VIII & + & - & - & - & + & 1 & - & \\
\hline TOTAL & & & & & & 83 & 30 & 10 \\
\hline
\end{tabular}

+ indicates growth on media lacking the particular amino acid.

model is that an $s-R N A$ species that normally recognizes nonsense is present but is not charged. The mutational event results in an alteration of the amino acid attachment site, allowing the s-RNA to become charged, and the amino acid to be inserted into the protein at the nonsense codon. With the observation of protein fragments in addition to complete proteins in a suppressor-bearing strain, the charging of the s-RNA is assumed to be incomplete. The above information leads us to propose a possible modification of the model involving $s-R N A$ to the effect that there exist redundant sets of $s-R N A$ genes, in which all members of a set make the same s-RNA. A mutational event occurring in one of the genes of a set would be reflected in the coding specificity (for normally charged s-RNA's) or in the ability to be charged (for normally uncharged $s-R_{N A}^{\prime} s$ ). In either case the altered $s-R N A$ is able to translate the nonsense codon by inserting the characteristic amino acid into the protein at that site. The remaining members of the set would function normally by translating their ordinary triplets.

This modification abrogates the necessity of a particular s-RNA coding both for nonsense and for its normal triplet. Since only one gene of a set is altered, the remaining members are normal and the mutational event is not lethal. The presence of protein fragments and complete proteins in a suppressor-bearing strain would be explained by the competition between the normal and mutant s-RNA. The observation of many phenotypic classes would be explained by the fact that amino acids inserted at the nonsense codon must be closely related to the normal amino acid for the protein to be functional; other insertions would form nonfunctional proteins. The occurrence of as many as six distinct suppressor loci (class I) that are indistinguishable phenotypically could indicate that all are members of one redundant set. Experiments on three other super-suppressors in yeast, $\underline{S}_{a}, \underline{S}_{b}$, and $\underline{S}_{d}$, Hawthorne and Mortimer (1) and Manney (2), have shown that over a wide range of alleles and loci tested 
these three are indistinguishable phenotypically, yet the three are at separate loci and $\underline{S}_{b}$ and $S_{d}$ have been shown to be different from the 10 suppressor loci discussed here.

\section{ACKNOWLEDGMENTS}

This research was sponsored by the U. S. Atomic Energy Commission and the U. S. Public Health Service under Biophysics Training Grant No. 5T 1 GM829.

\section{REFERENCES}

1. Hawthorne, D. C., and Mortimer, R. K.; Genetics 48: 617, 1963.

2. Manney, T. R.; Genetics 50:109, 1964.

3. Benzer, S., and Champe, S. P.; Proc. Natl. Acad. Sci. U. S. 48: 1114, 1962.

4. Garen, A., and Siddiqi, O.; Proc. Natl. Acad. Sci. U. S. 48: 1121, 1962.

5. Brody, S., and Yanofsky, C.; Proc. Natl. Acad. Sci. U. S. 50: 9, 1963.

6. Sarabhai, A. S.; Stretton, A. O. W.; Brenner, S., and Bolle, A.; Nature 201: 13, 1964.

7. Weigert, M. G., and Garen, A. ; Nature 206: 992, 1965.

8. Brenner, S.; Stretton, A. O. W., and Kaplan, S.; Nature 206: 994, 1965.

9. Capecchi, M. R., and Gussin, G. N.; Science 149: 417, 1965.

10. Eggertsson, G., and Adelberg, E. A.; Genetics 52:319, 1965.

11. Johnston, J. R., and Mortimer, R. K.; J. Bacteriol. 78: 292, 1959.

Received January, 1966. 


\title{
UV-Induced Dominant Lethality: Evidence for Different Lethal Systems between UV and X Irradiation in Saccharomyces
}

\author{
Robert L. Wiskocil
}

In previous studies $(1,2)$ concerning $X$-ray-induced dominant lethality in yeast, Mortimer suggested that chromosome bridges, aberrations, and mass deletions are possibly the cause of dominant lethal damage. Because ionizing radiations were known to cause such damage, it was suspected that $\mathrm{X}$ ray and not ultraviolet light would cause dominant lethality. Chu (3), however, discovered chromosome and chromatid aberrations and bridges that were induced by UV light in hamster cells. These chromosome aberrations were nonphotoreactivable and indistinguishable from those produced by ionizing radiations.

Mutagenic studies $(4,5)$ have shown a definite difference between UV light and X-rayinduced mutations. In bacteria, thymine dimers, which are praduced only by UV light, have been found to account for up to $70 \%$ of the inactivation of transforming DNA (6). Cytoplasmic "petites", leaky, complementing and point mutations are produced in significantly greater amounts by UV light than $\mathrm{X}$ irradiation. This difference between the mutagenic actions of $X$ ray and UV light would substantiate a suspected difference between the lethal systems of the two radiations. Elkind and Sutton $(7,8)$, however, have presented evidence for overlap in sites of lethal damage. By the use of UV-X-ray additivity experiments, they have found that the lethal effects of $X$ ray on budding cells can be mitigated by UV light and that, at high UV dose points, a pre-X-ray irradiation produces a curve that closely parallels the $X-r a y$ survival of budding cells. In view of these results, a comparative study between UV and X-rayinduced dominant lethality would be of considerable interest and relevance in a further comparison between sites of lethal action of UV and $\mathrm{X}$ irradiation.

\section{MATERIALS AND METHODS}

Haploid cells from the two tester cultures $52679 \mathrm{~B}$ and $52072 \mathrm{D}$ of the yeast Saccharomyces cerevisiae were used in the experiment. $52679 \mathrm{~B}$ had a genotype of a ad and $\mathrm{S} 2072 \mathrm{D}$ had the genotype $\underline{\alpha} \underline{l e}_{1} \underline{t r}_{1} t_{4} \underline{a r}_{4}$. The autodiploidized wild-type strain S288C with the mating type $\alpha \alpha$ was used along with the diploid strain, XB12 lobtained by mating cells from the haploid cultures). The haploid and $5288 \mathrm{C}$ strains were grown on YEPD medium (1\% yeast extract, $2 \%$ agar, $2 \%$ peptones and $2 \%$ dextrose) for $24 \mathrm{hr}$ at $30^{\circ} \mathrm{C}$. Immediately prior to irradiation, the cells from the cultures were transferred to sterile distilled water and suspended to a concentration of $\sim 10^{7}$ cells $/ \mathrm{ml}$. Cultures with only $24 \mathrm{hr}$ growth were used to insure good zygote formation. The procedure outlined by Lyman (9) was used to obtain a stationary growth phase. This technique was used in obtaining an interdivisionaI UV survival curve of the diploid strain XB12. 
Ultraviolet irradiation was performed with three tubular, low-pressure Hg lamps. The dose rate at the distance at which the cells were irradiated was $3.58 \times 10^{3} \mathrm{ergs} / \mathrm{sec} / \mathrm{cm}$. At this dosage, 95\% of the emitted energy was at 2,537.

A 10-watt General Electric visible light bulb was used to photoreactivate the cells for $3 \mathrm{hr}$ at a distance of $50 \mathrm{~cm}$. The temperature during the time of photoreactivation increased only $1^{\circ} \mathrm{C}$. For the dark experiment, a Kodak darkroom safelight with a yellow filter was used to insure the absence of photoreactivation light.

A study of UV-induced dominant lethals was performed as follows: A small loopful of cells from the $52679 \mathrm{~B}$ suspension was streaked across the surface of a YEPD agar slab, as described by Johnson and Mortimer (10). The cells were irradiated by UV under the conditions described above. After irradiation, a loopful of cells from a suspension of the opposite mating type was streaked over the irradiated cells. The slab was then placed in an agar-filled petri dish and incubated at $30^{\circ} \mathrm{C}$ for 4 to $5 \mathrm{hr}$. Of the zygotes thus formed, only those with diploid buds or without haploid buds were isolated (with a micromanipulator) from the general streak of cells. After the zygotes were isolated, the agar slab was placed on YEPD medium and incubated at $30^{\circ} \mathrm{C}$. Observations of the zygote clones were made at approximately 48 and $72 \mathrm{hr}$. The ability of a zygote to form a visible colony was used as a criterion of survival. Zygoteformation curves were obtained by employing the micropairing technique (11) on a YEPD slab. The survival of haploid budding and nonbudding cells was obtained by microscopic single-cell isolation on YEPD slabs.

\section{RESULTS}

When both parent cells were unirradiated, zygote formation was nearly $100 \%$ and viability was $100 \%$. However, with UV irradiation of one parent cell, the zygote formation was reduced. This inhibition was found to increase with dose, for example at doses of 20 and $40 \mathrm{sec}, 50$ and $10 \%$ of the pairs, respectively, formed zygotes. Mortimer (11) found that $z y-$ gote formation was nearly unaffected by $X$ irradiation. However, the zygote-formation inhibition seen in the present studies suggests that UV greatly affects the cytoplasm and/or the cell wall. Of the zygotes formed from one irradiated and one unirradiated cell, some failed to develop into visible colonies and were considered to contain a dominant lethal.

Many of the zygotes with a UV-induced dominant lethal formed a diploid bud, became swollen, and went through no further divisions; others formed colonies ranging from three to 25 cells before division stopped; and still others showed immediate signs of cell lysis. All of the above visible zygote damage was reported to exist in X-ray-induced dominant lethals (11). However, morphologically aberrant zygotes, significantly different from those produced by $X$ ray, were found to be caused by UV. With ultraviolet light many zygotes formed elongated diploid buds that were not found in zygotes receiving Xirradiation (see Figs. 1a and $1 \mathrm{~b}$ ). The zygote with an elongated bud did not, however, always contain a dominant lethal.

The surviving zygote clones were tested phenotypically, and many haploid colonies were found to arise from zygotes, one of whose parent cells was irradiated by 30 or more sec 

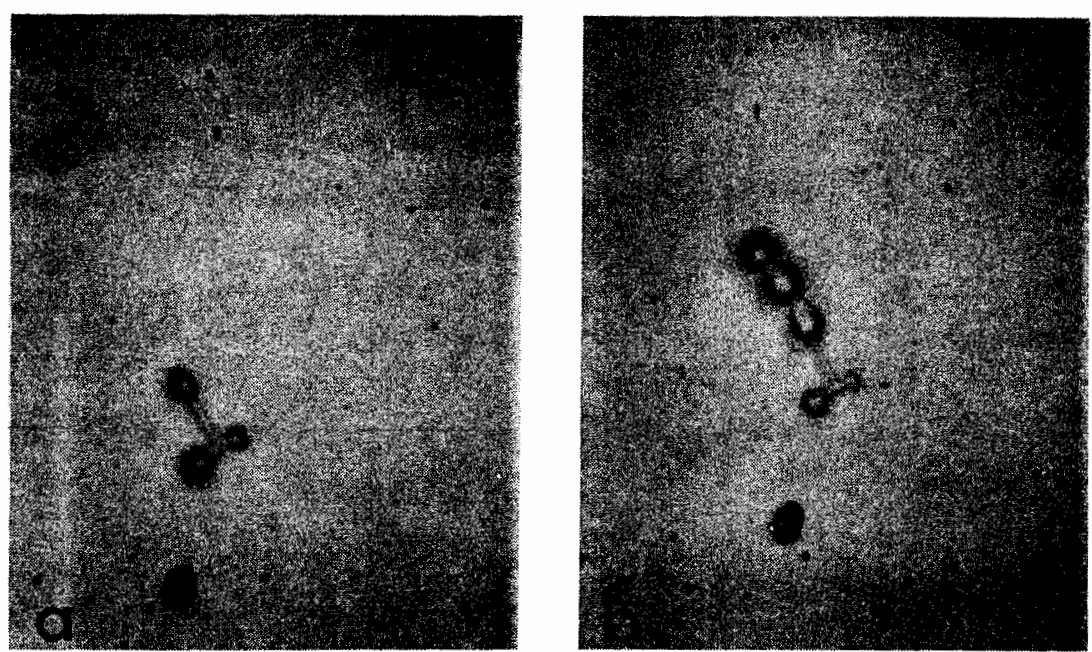

Figure 1. Photomicrograph of a diploid zygote that expressed a dominant lethal. This pattern was typical. a) Six hours after zygote formation; b) same zygote $24 \mathrm{hr}$ later. Note the lysis of the original zygote. No further division occurred within this clone.

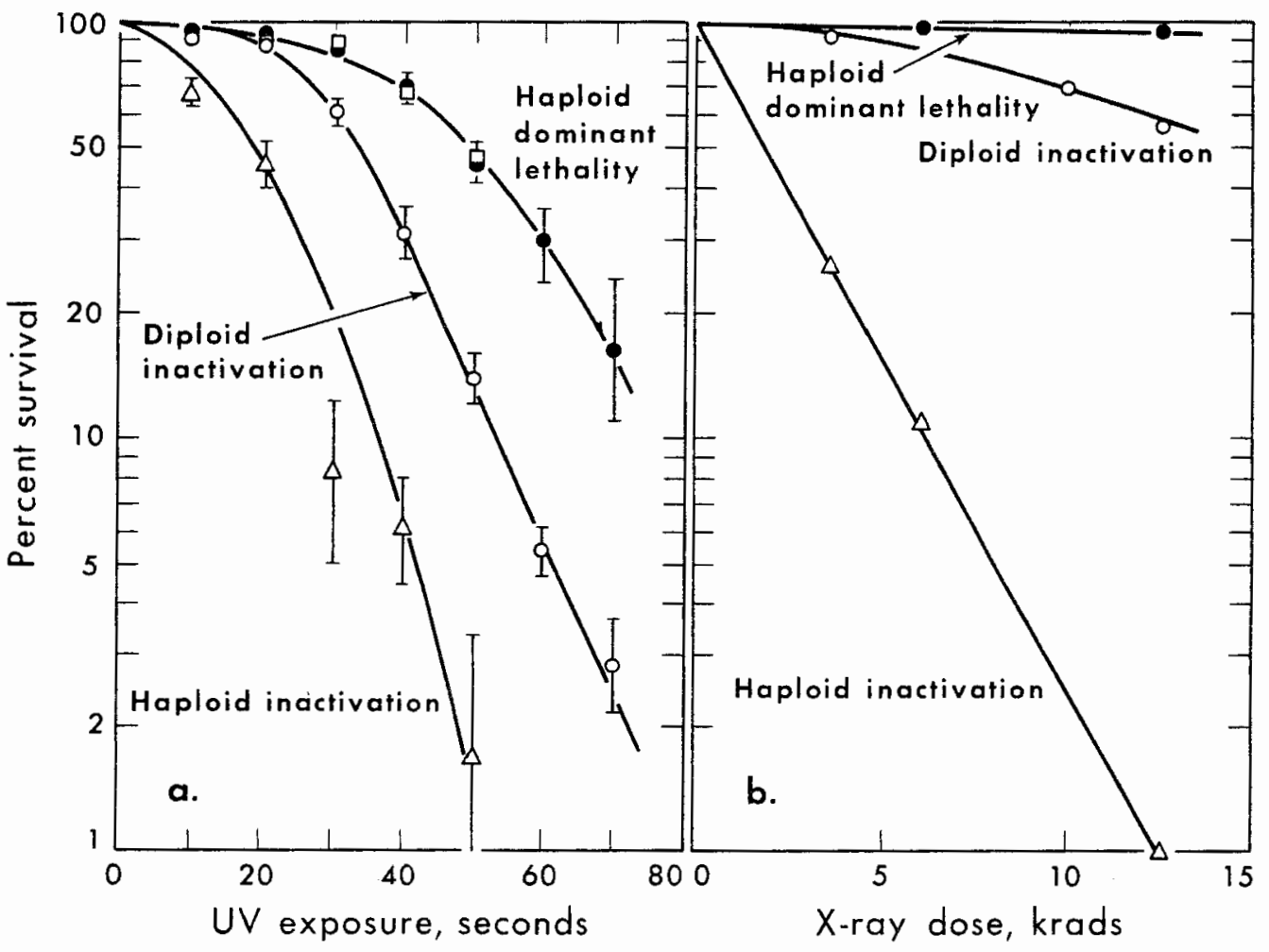

Figure 2. Survival and dominant lethal curves. The survival curves are for haploid and diploid cells. The dominant lethal curve is for zygotes formed from one irradiated and one unirradiated cell. a) Exposure to UV (this study); b) exposure to $\mathrm{X}$ rays (11).

$$
\text { MU. } 9600
$$


of UV. The haploid colonies always had the genotype of the unirradiated parent. This haploidization could be explained as a failure of the irradiated nucleus to unite with the unirradiated one.

In Fig. 2a are presented the survival curves for cell inactivation and induction of dominant lethals with UV. The corresponding curves for $X$ rays (11) are reproduced in $F i g .2 b$. The results indicate that dominant lethals account for a considerable portion of the UV-induced haploid inactivation. In comparing Figs. $2 a$ and $2 b$, it is apparent that the fraction of dominant lethals per haploid inactivation is greater with UV than with X irradiation. At $6 \%$ haploid survival, the ratio of dominant lethal damage to inactivation damage other than dominant lethality is 1:2 for UV and 1:46 for $X$ ray. In the case of both radiations, these ratios are not constant because the curves representing the two types of damage have different shapes.

There was no increase in zygote viability when the UV-irradiated parent was exposed to photoreactivating light. If UV-induced dominant lethality results from chromosome breaks and aberrations, then the fact that the dominant lethals are not photoreactivable is to be expected since such damage (UV-induced chromosome breaks) has been reported to be nonphotoreactivable (3).

\section{SUMMARY AND CONCLUSIONS}

In view of the data presented, the general conclusion can be stated that dominant lethals are produced in comparatively greater numbers by UV than by X irradiation. This difference in the production of dominant lethals gives a basis for assuming that the lethal lesions associated with the two radiations are also different. Elkind and Sutton (8) found that budding cells are less sensitive to UV than nonbudding cells. This relative difference of UV sensitivity, however, is considerably smaller than the difference in X-ray sensitivity between the budding and nonbudding.cells. Mortimer (5) demonstrated that only dominant lethals can inactivate dividing cells. The fact that UV inactivates a relatively greater number of budding cells than does $X$ ray substantiates the greater efficiency of UV light in producing dominant lethais.

With the use of additivity experiments, Uretz (12) concluded that there was a definite overlap in lethal sites of UV and X ray. Because there was not a reciprocal response between pre-UV and pre-X-ray treatment of diploid cells, it can be said that the overlap is only partial. Partial overlapping of sites, however, does not discount the theory that there are definite differences between the lethal systems of UV and X ray.

Diploid bud elongations as well as a zygote formation inhibition effect were found to be caused only by UV irradiation. Although the damage is not necessarily lethal, these effects illustrate further differences between ultraviolet-and $\mathrm{X}$-ray induced damage.

\section{ACKNOWLEDGMENTS}

I wish to thank Professor Robert $K$. Mortimer for his constant interest and advice which have contributed greatly to the experiment. Discussions with Richard Gilmore, Mike Resnick, and Tommy McKey have been beneficial. 
This work was supported by the State of California Cancer Grant to the University of California.

\section{REFERENCES AND NOTES}

1. Mortimer, R. K. F Factors Controlling the Radiosensitivity of Yeast Cells, Brookhaven Symposia in Biol. No. 14, 1961.

2. Mortimer, R. K.; Rad. Res, 9:312-326, 1958.

3. Chu, H. Y.; Mutation Res. 2:75-94, 1965.

4. DeSerres, F. J.; J. Cell. and Comp. Physiol. 64 Suppl. 1: 33-42, 1964.

5. Mortimer, R. K.; Radiation Res. Suppl. 1: 394-402, 1959.

6. Setlow, R. B., and Setlow, J, K.; Proc. Natl. Acad. Sci., U. S. 48: 1250-1257, 1962.

7. Elkind, M. M., and Suttor, H.; Radiation Res. 10: 296-312, 1959.

8. Elkind, M. M., and Sutton, H.; Radiation Res. 10: 283-295, 1959.

9. Lyman, J. T.; Ph. D. Thesis, University of California, Lawrence Radiation Laboratory Report UCRL-16030, 1965.

10. Johnson, J. R., and Mortimer, R. K.; J. Bactriol. 78:292, 1959.

11. Mortimer, R. K.; Radiation Res. 2: 361-368, 1955.

12. Uretz, R. B.; Radiation Res. 2: 240-252, 1955.

Robert L. Wiskocil is a student at The Choate School, Wallingford, Conn.

Received November, 1965. 


\title{
The Gene-Interaction Component of the Genetic Load
}

\author{
Jack Lester King
}

In the continuing discussions concerning the relative magnitude of mutational and balanced components of genetic load, the interaction component has received relatively little attention. It is the purpose of this article to propose that the interaction component is a major part of the concealed genetic load, and to explore the implications of certain types of genetic interaction.

Deleterious alleles, with the exceptions of completely dominant and completely recessive lethals, are variable in expression with regard to fitness. They contribute to the reproductive extinction of some carriers (or potential carriers) and not of others. In most cases this variability of expression probably has both genetic and nongenetic components, i.e., gene interaction is probably common in genomes eliminated by selection. When two or more deleterious alleles interact to affect the fitness of a carrier more severely than the sum of their individual average effects, the extra deleterious effect is an expression of a formerly concealed portion of the genetic load.

\section{MUTATION AND THE "GENETIC DEATH" RATE}

Haldane (1) and Muller (2) concluded that the reduction in fitness of a population caused by mutation was approximately twice the sum of all mutation rates and independent of the severity of expression of the individual dominant or recessive mutations; and that approximately one individual, on the average, is eliminated for every dominant mutation and for every two fully recessive mutations. Most subsequent theoretical estimations of total mutational damage have been based on this premise. If the interaction component is a major part of the genetic load as proposed, however, the premise as stated does not hold. Muller was aware of this and suggested that more experimental work on the extent of synergis $m$ was needed.

The occurrence of those "genetic deaths" caused by the collaboration of a number of harmful genes is approximately equal to twice the average mutation rate of the genes involved, rather than approximately twice the total of the mutation rates. Mutations with relatively slight effects are likely to be expressed and lost only in conjunction with other deleterious alleles, and thus contribute relatively less to the expressed genetic load.

\section{PROOF}

In a genetic system with $2 \mathrm{~N}$ (diploid) loci, $2 \mathrm{~N} \overline{\mathrm{q}}$ is the mean number of deleterious mutant alleles carried per adult; $2 \mathrm{~N} \bar{f}$ is the mean number of such alleles per genome in the 
class of genomes eliminated by selection; $2 N \mu(1-q)$ is the total number of mutations in the system per diploid genome per generation; $D$ is the rate of the selective elimination of genomes (Muller's "genetic death" rate; Haldane's mean loss of fitness) attributable to the system. Then at equilibrium

$$
2 N \bar{q}=\frac{2 N \bar{q}-D \bar{f}+2 N \mu(\overline{1-q})}{1-\bar{D}} .
$$

The genetic death rate is:

$$
D=\frac{\mu(\overline{1-q})}{\bar{f}-\bar{q}}=\frac{2 N \mu(\overline{1-q})}{2 N \bar{f}-2 N \bar{q}} .
$$

If $2 \mathrm{Nq}$ is small relative to $2 \mathrm{~N} \overline{\mathrm{f}}$, the genetic death rate is approximately twice the average mutation rate of the average number of deleterious alleles involved in each selection event. When homozygous alleles are involved, the mutation rate is counted only once.

If $q_{i}$ is the gene frequency at the ith locus in the surviving population, and $f_{i}$ is the gene frequency among the individuals selected against, the selection coefficient (the probability that a given gene will be eliminated by selection in one generation) is

$$
s_{i}=\frac{D f_{i}}{D_{i}+q_{i}(1-D)} \text {. }
$$

$D$ and $f_{i}$ are partially dependent on $q_{i}$, but for a given value of $D$ the equilibrium frequency at the ith locus is

$$
\hat{q}_{i}=\frac{D f_{i}=\mu_{i}}{D-\mu_{i}} \text {, or } \hat{q}_{i}=1-\frac{D\left(f_{i}-q_{i}\right)}{\mu_{i}} \text {. }
$$

\section{THRESHOLD SYSTEMS}

It is well known that some systems involve the interactions of many genes. Threshold systems are characterized by the stability of the phenotype (or one aspect of it) over a broad range of genetic and environmental variability, with a discontinuity when the genetic and environmental factors pass beyond a limit of allowable variation. Although almost all empirical studies of threshold systems have dealt with visible morphological characters, other components of fitness must behave genetically in the same way.

Actually, any component of fitness in which gene interaction occurs can be considered as a threshold character. Death or sterility are the transthreshold phenotypes. In cases of reduced fecundity or material ability, it can be considered that the threshold applies to the offspring or potential offspring, the transthreshold group being those that fail to survive or fail to be conceived. The genomes selected against are those of the real or potential offspring, of which only a portion may carry the harmful genotypes.

A genetic threshold system was analyzed by Wright (3). Following Wright one may conveniently consider threshold systems in terms of a normally distributed continuous 
parameter that has nongenetic and additive genetic components of variance. Each of the array of alleles available at each locus contributes additively to the parameter. Those that contribute more than the average value for the locus can be considered to be "plus" alleles; "minus" alleles either contribute nothing, or less than the average value for the locus in the population. Individuals for which the total value of the parameter falls below a minimum threshold are eliminated by selections.

It is important to emphasize that the parameter is an intellectual construction describing the developmental and population genetic behavior of threshold system and does not necessarily describe any real thing or substance in the developing organism. The normality of the parameter and the additivity of the contributing genes are convenient first order approximations, pending better understanding of developmental mechanisms. The threshold is at $\mathrm{z}$ standard deviations below the mean of the distribution; the genetic death rate $D$ is equal to the area of the unit normal distribution below $-z \sigma$, or $\mathrm{D}=\mathrm{A}_{(\mathrm{z})^{\circ}}$. In such a system deleterious alleles are lost in clusters, and $2 \mathrm{~N} \bar{f}-2 \mathrm{~N} \bar{q}$ may conceivably be large. If such systems are numerous, the total threshold component of the genetic load could be as important as components attributable to independently acting genes. The interaction component of the genetic load is part of the mutational load, in that the deleterious alleles are maintained in the population through mutation pressure. At the same time the interaction component is closely related to the segregational load, since the deleterious alleles are expressed only when clustered by chance segregation.

\section{SYNERGISM IN THRESHOLD SYSTEMS}

If a minus allele of a threshold system is tested in the laboratory, its carriers will be found to have a fitness value of less than one. The mean of the continuous parameter in the population of known carriers is shifted toward the threshold which is tantamount to shifting the threshold toward the mean. If $e_{1}$ is the effect of the known allele in standard deviations, the rate of transthreshold eliminations among known carriers is equal to the area of the unit normal distribution below $-\left(z-e_{1}\right)$. The fitness value derived by empirical observation would be:

$$
1-s_{1}=\frac{1-A_{\left(z-e_{1}\right)}}{1-A_{(z)}} \text {. }
$$

A second allele from the same system, tested separately, would have an empirical fitness value of $1-s_{2}$. The two alleles together, under the hypothesis of independent action, would be expected to exhibit a fitness equal to the product of their individual fitnesses: $\left(1-s_{1}\right)$ $\left(1-s_{2}\right)$. Instead, although their direct contributions to the continuous parameter are additive and independent, they act synergistically with respect to fitness because of the shape of the curve, Fraser (4), to produce a combined fitness value of:

$$
1-s_{1+2}=\frac{1-A_{\left(z-e_{1}-e_{2}\right)}}{1-A_{(z)}} \text {. See Fig. } 1 .
$$

Additivity is the simplifying assumption, but individual loci might be dominant or recessive with respect to their contribution to the parameter. Synergism as described above can occur between two homozygous loci. On the other hand, deleterious alleles that are strictly additive in their contributions to the parameter would show a degree of recessivity with regard to fitness because of synergism between homologues. 


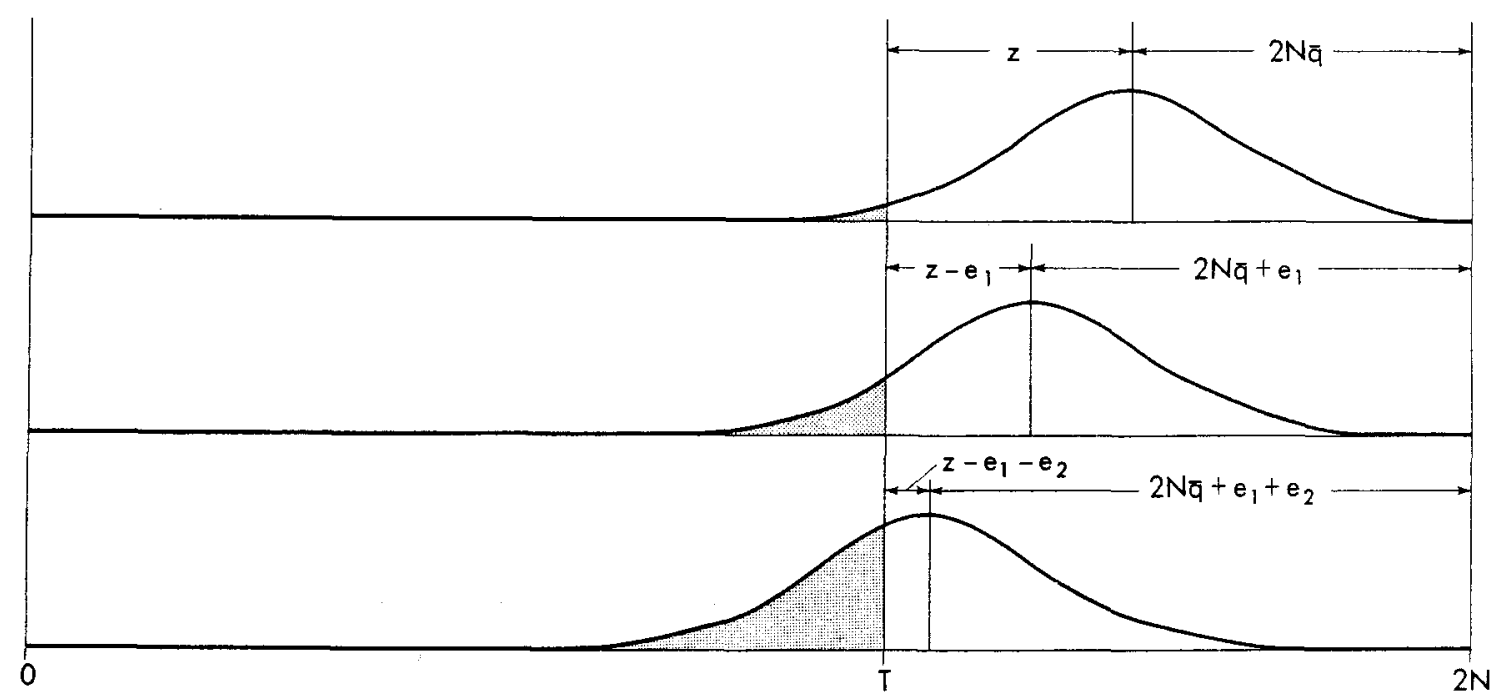

Figure 1. Synergism in a threshold system. Top: Distribution and death rate in an outbred population. Center: Distribution and death rate in the class of individuals carrying an identified deleterious allele, which displaces the mean $e_{1}$ standard deviations. Bottom: Distribution and death rate in the class of individuals carrying two identified deleterious alleles, which displace the mean $e_{1}$ and $e_{2}$ standard deviations respectively. The fundamental action of the two deleterious alleles is additive with respect to the parameter, but the effect on the genetic death rate is synergistic.

MUB-7609

\section{TWO-THRESHOLD SYSTEMS}

In single threshold systems, the gene equilibrium at each locus is near fixation for the plus allele, at a point where the loss of minus alleles through threshold selection is balanced by mutation pressure. In canalized systems, Waddington (5) and Rendel (6), there is an upper as well as a lower threshold, so that selective losses occur for high as well as for low values of the parameter. High threshold selection removes from the population individuals with fewer minus alleles than the population average, thereby tending to increase the frequency of minus alleles in the surviving population. High threshold selection is thus added to mutation pressure to increase the equilibrium value of $q_{i}$. If mutation from minus to plus is negligible, the mathematical relationship between the mutation and genetic death rates is as previously formulated; however, genomes eliminated at the upper threshold, having on the average fewer than $2 N \bar{q}$ minus alleles, make negative contributions to $2 N \bar{f}-2 N \bar{q}$. In effect, however, a portion of the genetic load is not due directly to mutation but is a balanced load attributable to the opposing actions of high and low thresholds.

Individual loci in a canalized system may stabilize near fixation for either the plus or the minus allele, so that neither minus nor plus can be considered to be abnormal or mutant in the system. If the system is fairly symmetrical, mutation from minus to plus must be considered. Such a canalized system has two subsets of loci in the population, those near fixation for plus and those near fixation for minus. Selection at the high threshold primarily affects the mostly minus subset, and balances the mutation pressure of minus-to-pius in that 
group. Low threshold selection balances the plus-to-minus mutation pressure of the mostly plus subset. The subsets could conceivably overlap, with the third group near fixation for alleles with intermediate values and subject to mutation pressure in both directions. In many respects a canalized system near fixation at all loci would behave as two single threshold systems. Any shift in the mean of the parameter, however, would simultaneously intensify selection at one threshold and relax it at the other.

When $q_{i}$ has an intermediate value in a two-threshold system, the heterozygote has a selective advantage over either homozygote. This is because heterozygosis is less likely than homozygosis to occur in the transthreshold genomes. If mutation pressure, outbreeding, or other factors are able to sustain appreciable polymorphism in canalized systems, this type of heterosis would be quite significant. Polymorphism in canalized systems might be responsible for the observations that led Lerner (7) to propose the developmental necessity of obligate levels of heterozygosity, and for other indications of the widespread occurrence of heterosis in natural populations.

It has been argued, e.g., Crow (8), that the number of heterotic loci must be small in any population, since the loss of fitness necessary to keep very many loci polymorphic would be far too great. In canalized polygenic systems, however, the loss of fitness is slight relative to the sum of the selective disadvantages of homozygosis, since a cluster of homozygous alleles is lost with each "genetic death". Furthermore, the heterozygote advantage in such systems is not the cause of such polymorphism as does exist, but rather the result of it.

It might be thought that a heterozygote advantage would automatically assure polymorphism, but this is not the case. Single-locus heterosis tends to favor the increase of the rarer allele and thus to achieve a stable polymorphism, because selection against either allele is directly proportional to the frequency of the homozygote. In polygenic threshold systems, however, the number of homozygotes eliminated at each threshold remains stable over the very range of frequencies at which there is a heterozygote advantage. Selection which removes a fixed number of plus and minus homozygotes has exactly the same effect as selection against heterozygotes: the gene frequency is driven away from an unstable equilibrium point toward fixation at either extreme. Thus, two-threshold systems, in the absence of mutation or other disturbing effects, tend toward fixation of every locus, Robertson (9), at some value that may be either minus or plus, relative to the original mean value of the locus. However, this tendency is extremely slow even under those hypothetical conditions, Fraser (10), although random fixation is likely to occur in small populations.

\section{ALLELE FREQUENCY EQUILIBRIA}

If the mutation rate from minus to plus is $v$, the change in frequency of the minus allele per generation at the ith locus is $\Delta q_{i}$ :

$$
q_{i}+\Delta q_{i}=\frac{q_{i}-D f_{i}+\mu_{i}\left(1-q_{i}\right)-q_{i} v_{i}}{1-D} ;
$$




$$
\Delta q_{i}=\frac{q_{i}\left(D-\mu_{i}-\nu_{i}\right)+\mu_{i}-D f_{i}}{1-D} .
$$

At equilibrium $\Delta q_{i}=0$ and

$$
\hat{q}_{i}=\frac{D f_{i}-\mu_{i}}{D i-\mu_{i}-\nu_{i}} .
$$

The values of $f_{i}, q_{i}$ and $D$ are interdependent and dependent on other gene frequencies, but stable equilibria exist somewhere near $q_{i}=0$ and $q_{i}=1$; there is also an intermediate, unstable equilibrium point. In the absence of mutation, $\hat{q}_{i}=f_{i}$, which occurs when both are either one or zero.

Threshold systems do allow for the extended survival of genetic variability arising from mutation pressure and fluctuating environment. Changes in the distribution of the parameter are met with compensating genetic responses which can include the rapid increase in a formerly rare allele. The genetic variability of threshold systems exists in an extremely flexible, responsive state of dynamic disequilibrium, since all gene frequencies in each system are interdependent. If some of the constituent genes are pleiotropic, acting in several threshold systems, the systems are interdependent also.

In a highly relevant study Magalhães et al. (11) introduced recessive lethals in high frequencies into caged Drosophila willistoni populations. Sampling at later generations, they found that the "lethal" alleles had persisted in the populations and had acquired linked suppressors, having thus become less deleterious. The populations had made a compensatory genetic response to the presence of the "lethal" alleles. In the terminology of the present discussion, Magalhães' introduction of the lethals shifted the population mean of the continuous parameter toward one threshold; natural selection rapidly shifted it back through the selective elimination not only of the introduced allele but also of other alleles with similar fundamental activities. The result was a selective increase of the alleles acting in an opposing direction. These Magaihães' et al. quite properly term "suppressors."

This genetic responsiveness, or genetic homeostasis (7), in allowing utilization of concealed variability in prompt responses to changes in the environment or gene pool, could also allow a recurrent mutation with favorable and unfavorable pleiotropic effects to become incorporated into the gene pool even though its net effect on fitness had been negative.

An important corollary is that an upset in the balance of threshold systems by the sudden introduction of a load of induced mutations can be substantially rectified within a very few generations, although the mutations themselves may persist indefinitely. This is extremely pertinent to the empirical estimation of total mutation damage caused by radiation and other mutagens. 


\section{INBREEDING DEPRESSION AND THRESHOLD SYSTEMS}

The concealed genetic loads attributable to mutation pressure and balanced heterosis are in the form of deleterious recessives, which may be revealed by inbreeding. Although the concealed genetic load of threshold systems does not necessarily involve deleterious re cessives, it too can be revealed by inbreeding, Newcombe (12). Much of the depression of vigor and viability associated with inbreeding is probably due to threshold effects, Wright (13) and Sheldon et al. (14). Inbreeding in a previously outbred population increases the genetic component of variance, and therefore the total proportion of transthreshold values for both high and low threshold is also increased (Fig. $2(8,12)$ ).

The variance of the continuous parameter $\sigma_{\mathrm{T}}^{2}$ has two principal components, genetic and nongenetic: $\sigma_{g}^{2}$ and $\sigma_{c}^{2}$ respectively. A degree of inbreeding $F$ increases the additive genetic component of variance by $1+F$, Robertson (15), and raises the total variance of the parameter from $\sigma_{\mathrm{T}}^{2}$ to $\left(\sigma_{\mathrm{T}}^{2}+F \sigma_{\mathrm{g}}^{2}\right)$. The threshold remains at $-z \sigma_{\mathrm{T}}$. Since the standard deviation of the parameter is increased, however, the equivalent threshold on the unit normal distribution is at $-z^{\prime}$, where

$$
-z^{\prime}=z \sqrt{\frac{\sigma_{T}^{2}}{\sigma_{T}^{2}+F \sigma_{g}^{2}}} .
$$

If the nongenetic component of variance is negligible, or if it remains proportional to the genetic variance, then

$$
z^{1}=z(1+F)^{-1 / 2}
$$

The proportion of lethal or deviant phenotypes caused by threshold systems increases rapidly and nonlinearly with $F$ (Table 1 and Fig. 3). If there is recessivity at any of the loci in a system, the increases and the nonlinearity are even more marked (8). Empirical studies have revealed nonlinear relationships between inbreeding depression and $F$ (Dobzhansky et al. (16), Levene et al. (17), Malogolowkin-Cohen et al. (18).

\section{INBRED LINES AND HYBRID VIGOR}

Continued inbreeding stabilizes genotypes and greatly reduces the genetic variance within lines. Threshold systems may become stabilized near their optima, so that no further losses occur from them. Some systems, however, will become genetically fixed near enough to one of their threshold values that nongenetic variation continues to cause a substantial number of subthreshold individuals. Fitness is reduced and phenotypic variability is increased (13), although the total distance between canalized thresholds is not altered (14). Hybrids between separately obtained lines show none of this reduction in fitness unless one or more of the same systems happen to have become fixed near their thresholds in both lines. Because more outbred small populations are subject to random fixation and wide fluctuations in the genetic variability of threshold systems, crosses between different populations are also apt to exhibit hybrid vigor. 


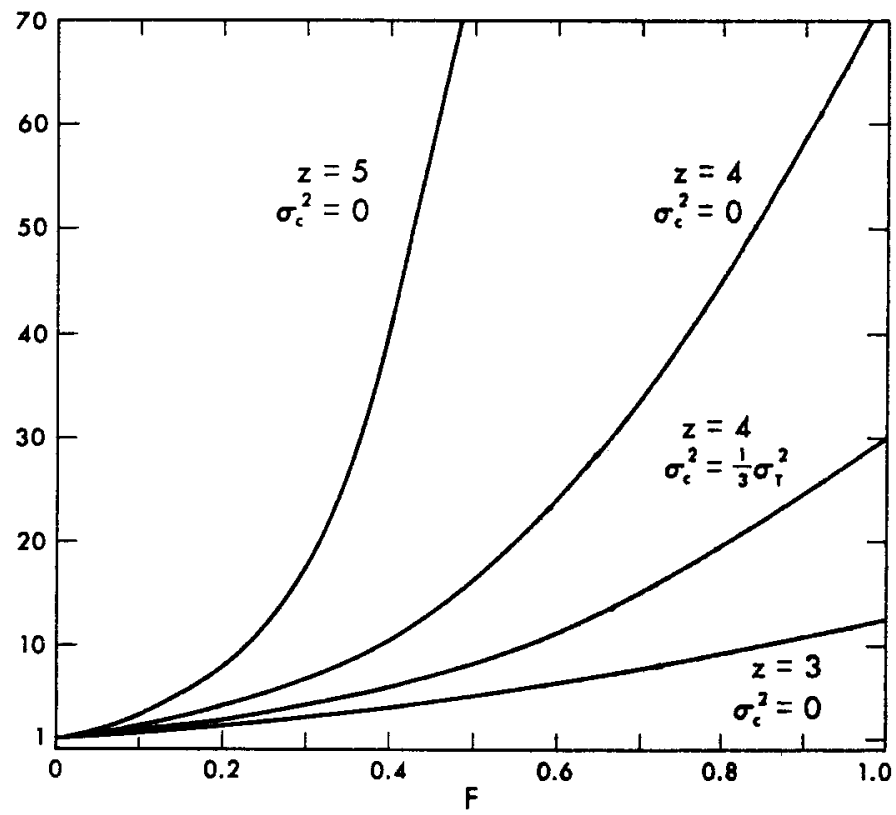

Figure 2. Relative increases in genetic death rate with increases in degree of inbreeding ( $F)$. The genetic death rate of each of four hypothetical examples is taken as one with outbreeding. The genetic death rates are nonlinear with $F$. See Table 1. MUB-7671

Figure 3. Increased genetic variance causes an increase in the proportion of the distribution which falls above and below the two thresholds of a canalized system (not to scale). MUB-7607

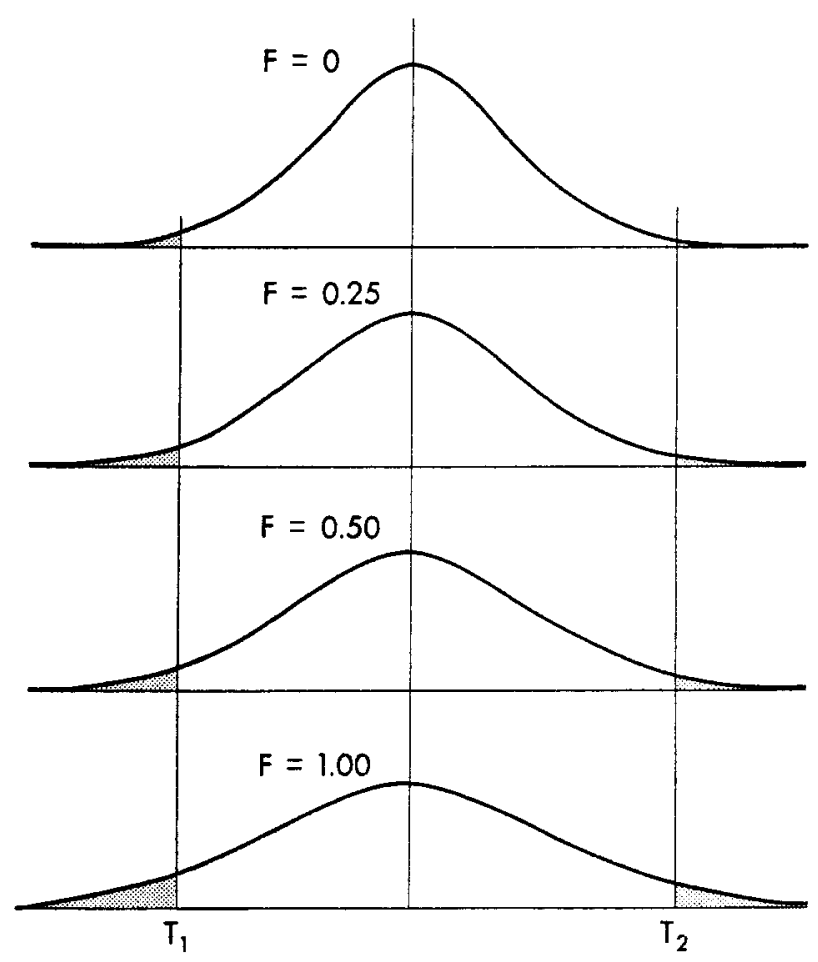


Table 1. The effect of inbreeding ( $F)$ on the frequencies of lethal or deviant phenotypes attributable to threshold systems

\begin{tabular}{llllllll}
\hline Threshold & $\begin{array}{l}\text { Nongenetic } \\
\text { variance }\end{array}$ & $F=0$ & $F=0.125$ & $F=0.25$ & $F=0.50$ & $F=0.75$ & $F=1.00$ \\
\hline (1) $z=3 \sigma_{T}$ & $\sigma_{c}^{2}=0$ & 0.00135 & 0.00233 & 0.00368 & 0.00734 & 0.0117 & 0.0170 \\
$(2) z=4 \sigma_{T}$ & $\sigma_{c}^{2}=0$ & $3.17 \times 10^{-5}$ & $9.15 \times 10^{-5}$ & $17.9 \times 10^{-5}$ & $54.7 \times 10^{-5}$ & $123 \times 10^{-5}$ & $232 \times 10^{-5}$ \\
(3) $z=4 \sigma_{T}$ & $\sigma_{c}^{2}=\frac{1}{3} \sigma_{T}^{2}$ & $3.17 \times 10^{-5}$ & $5.91 \times 10^{-5}$ & $10.78 \times 10^{-5}$ & $26.0 \times 10^{-5}$ & $54.7 \times 10^{-5}$ & $96.8 \times 10^{-5}$ \\
(4) $z=4 \sigma_{T}$ & $\sigma_{c}^{2}=\frac{1}{2} \sigma_{T}^{2}$ & $3.17 \times 10^{-5}$ & $5.22 \times 10^{-5}$ & $9.15 \times 10^{-5}$ & $17.9 \times 10^{-5}$ & $31.4 \times 10^{-5}$ & $54.7 \times 10^{-5}$ \\
(5) $z=5 \sigma_{T}$ & $\sigma_{\mathrm{C}}^{2}=0$ & $0.0287 \times 10^{-5}$ & $0.118 \times 10^{-5}$ & $0.41 \times 10^{-5}$ & $2.25 \times 10^{-5}$ & $7.84 \times 10^{-5}$ & $20.8 \times 10^{-5}$
\end{tabular}

The nongenetic component of variance in each of the first, second and fifth examples is either negligible or remains proportional to the genetic variance. In the third and fourth examples, the nongenetic components of variance are constants equal to one third and one half the total variance of the outbred population, respectively.

\section{SUMMARY}

The long -accepted premise that the total damage done to a population by a mutation is unrelated to the severity of the effect on individual carriers, and that an average of approximately one individual is lost by selection for every dominant mutation is incorrect. The rate of occurrence of those "genetic deaths" caused by the collaboration of a number of harmful genes is approximately equal to twice the average, rather than twice the total, of their mutation rates. Previous estimates of the total effect of mutation damage should be reduced. A major portion of the concealed genetic load of natural populations may be the interaction component. Deleterious alleles are introduced into the gene pool by mutation and expressed, in clusters, through chance segregation. A portion of the expressed genetic load attributable to canalized systems is not caused directly to mutation, but to opposing actions of the high and low thresholds. Heterozygotes for loci in canalized systems have a selective advantage over an intermediate range of gene frequencies, but this does not lead to balanced polymorphism; in the absence of mutation, all loci would tend toward fixation. Loci in canalized or twothreshold systems may be kept in fluctuating polymorphic disequilibria through the interactions of threshold selection, mutation, pleiotropy and environmental change, because gene frequencies at interacting loci are responsively interdependent. This genetic flexibility allows for great adaptability. An upset of the balance of threshold systems by a sudden load of induced mutation can be rectified in a few generations by compensatory genetic responses, although the mutations themselves may persist indefinitely. Inbreeding increases the genetic variance, and thus the proportion of transthreshold individuals. Whether deleterious alleles are additive or recessive, the predicted inbreeding depression caused by threshold systems rises rapidly and nonlinearly with the degree of inbreeding, $F$.

\section{ACKNOWLEDGMENTS}

I have been privileged to correspond with Everett Dempster, Theodosius Dobzhansky, F. Clark Fraser, Howard B. Newcombe and Sewall Wright, receiving suggestions and helpful comments from each. 
This investigation was supported in part by a postdoctorai fellowship from the National Institute of Arthritis and Metabolic Diseases and in part by the U. S. Atomic Energy Commission.

\section{REFERENCES}

1. Haldane, J. B. S.; Am. Naturalist 71:337-349, 1937.

2. Muller, H. J.; Am. J. Human Genet. 2: 111-176, 1950.

3. Wright, S.; Genetics 19: 537-551, 1934.

4. Fraser, F. C. ; in Teratology, edited by Wilson and Warkany, Chicago, U. Chicago Press, 1965, Chpt. 2.

5. Waddington, C. H.; The Strategy of the Genes, London, George Allen and Unwin, Ltd., 1957.

6. Rendel, J. M. R.; Evolution 13:425-439, 1959.

7. Lerner, I, M.; Genetic Homeostasis, New York, Wiley, 1954.

8. Crow, J. F.; Population genetics studies, in Second International Conference on Congenital Malformations, New York, International Medical Congress Ltd. , 1964.

9. Robertson, A.; J. Genet. 54:236-248, 1956.

10. Fraser, A. S.; J. Theoret. Biol. 2:329-346, 1962.

11. Magalhães, I. E.; Brito da Cunha, A.; De Toledo, J. S.; Toledo F, S. A.; De Souza, H. L.; Targa, H. J.; Setzer, V., and Pavan, C.; Mutation Res. 2: 45-54, 1965.

12. Newcombe, H. B.; Panel Discussion of the Session on Epidemiologic Studies, in Second International Conference on Congenital Malformations, New York, International Medical Congress Ltd., 1964.

13. Wright, S.; Genetics 19:471-505, 1934.

14. Sheldon, B. I.; Rendel, J. M., and Finlay, D. E.; Genetics 49: 471-484, 1964.

15. Robertson, A.; Genetics 37:189-207, 1952.

16. Dobzhansky, Th.; Spassky, B., and Tidwell, T.; Genetics 48: 361-373, 1963.

17. Levene, H.; Lerner, I. M.; Sokoloff, A.; Ho, F. K., and Franklin, I. R. ; Proc. Nat1. Acad. Sci. 53:1042-1050, 1965.

18. Malogolowkin-Cohen, Ch.; Levene, H.; Dobzhansky, N. P., and Solima Simmons, A.; Genetics 50: 1299-1311, 1964.

Received December, 1965. 


\title{
Electrophoretic Studies of Light-Induced Charge in Spinach Chloroplasts
}

\author{
Park S. Nobel and Howard C. Mel
}

The absorption of light by chloroplasts has been implicated in electrical changes of photosynthetic organisms. Light-induced membrane potential changes for whole plant cells have been known since the 1930's (1). More recently, Nagai and Tazawa (2), using microelectrodes, have reported that the potential of internodal Nitella cells becomes more negative in the light. Green light did not change the resting potential, while red and blue light did, suggesting that chloroplasts were important for this change. (However, light-dependent electrical changes of isolated chloroplasts have not previously been investigated directly.)

Nishizaki (1) has pointed out one complicating factor: both the magnitude and the direction of the potential change depend on the preillumination conditions.

Electrophoretic studies on cells have thus far failed to demonstrate a relation between membrane potential changes and net charge changes. Nevertheless, because of the specialized energy-conversion function of chloroplasts, it seemed worthwhile to investigate the possibility of direct, light-induced net charge effects. In this study we report electrophoretic mobilities of isolated spinach chloroplasts, measured in the light as well as in the dark, and discuss certain metabolic implications of the change observed.

\section{MATERIALS AND METHODS}

Spinach was purchased commercially and stored at $4^{\circ} \mathrm{C}$ until use. Leaves were rinsed with water, their midribs removed, and approximately $50 \mathrm{~g}$ of leaf material added to $100 \mathrm{ml}$ of isolation medium in a Waring Blendor and homogenized for $10 \mathrm{sec}$. The isolation medium is described at the end of this section. The homogenate was filtered through four layers of cheesecloth, and the filtrate was centrifuged in the cold in $40-\mathrm{ml}$ Servall centrifuge tubes for $11 / 2 \mathrm{~min}$ at $200 \mathrm{~g}$. The supernatant fraction was removed and centrifuged for $8 \mathrm{~min}$ at $200 \mathrm{~g}$ to form the chloroplast-containing pellet. The wet pellet was dispersed without addition of isolation medium and then filtered through a fine-mesh nylon bag to remove large debris. This method of isolation gave a higher percentage of whole chloroplasts than did other methods tried. The percentage of whole chloroplasts was judged by the height of the peak at $55 \mu^{3}$ in a Coulter Counter as well as by observation in a light microscope. Chlorophyll was measured spectrophotometrically (3); the number of chloroplasts/mg of chlorophyll was $15 \pm 1 \times 10^{8}$ as determined by the Coulter Counter and checked by a hemocytometer. In all experiments the preparation was diluted to a chloroplast concentration of $10^{7} / \mathrm{ml}$, corre sponding to $7 \mu \mathrm{g}$ of chlorophyll/ml. 
The chloroplast sample was subjected to stable-flow free-boundary (STAF LO) electrophoresis (4) and a steady-state migration pattern established (see Fig. 1). In this flow system the horizontal dimension becomes equivalent to a time axis. Thus, in the presence of a vertical electric field the average slope of the migration pattern should be proportional to particle electrophoretic mobility (4). " The pattern can be established in the dark as well as in the light, and observations made sufficiently rapidly so as not to change the "dark" pattern. In ordinary microelectrophoresis the light required for observations could be expected to preclude such "dark" measurements.

The STAFLO apparatus employed (Fig. 1) had a main migration chamber $30 \mathrm{~cm}$ long, $3 \mathrm{~cm}$ high, and $0.7 \mathrm{~cm}$ wide, with 12 symmetrical inlet and outlet channels. Reading from top to bottom, solutions continuously pumped into the 12 channels contained the following weight percentages of sucrose: $0.0,0.0,0.1,0.2,0.3,0.4,0.5,0.6,0.7,0.8,3.0$, and 3.0. A cellophane (dialysis tubing) membrane serves as a high hydrodynamic yet low electrical resistance isolation barrier between the migration chamber and the electrode compartments. A design change in the platinum-electrode chamber now permits electrophoresis at currents in excess of $100 \mathrm{~mA}$ (0.5 watts), useful for work at higher ionic strengths. When the original 1-cm-high electrode chamber is reduced to $0.20 \mathrm{~cm}$ in height, the linear flow velocity for the $20 \mathrm{ml} / \mathrm{min}$ electrode-fluid flow rate is sufficiently rapid to wash out the gas bubbles formed at the irreversible electrodes and to give rise to a time-averaged undisturbed field. This rapid electrode fluid flow also serves to remove heat and to suppress migration of electrolysis products into the main chamber.

The small density (composition) gradients customarily employed in ST AF LO experiments have proved useful in minimizing interference of the gradient-forming substance with metabolic processes in chloroplasts. With such small gradients, however, even the slight temperature rise from Joule heating (for these experiments, usually less than $0.5^{\circ} \mathrm{C}$ from inlet to outlet) is sufficient to cause a detectable distortion in the migration pattern (see Run 5, Fig. 2). Correction was made for this, and simultaneously for the slight chloroplast sedimentation, by the following control: the migration pattern was observed while the electric field was on continuously for $60 \mathrm{~min}$ but the polarity periodically reversed, e.g., every $2 \mathrm{~min}$. (The heating effect does not depend on the direction of the electric field.) The reported mobilities have been corrected point by point for this effect.

The chloroplast suspension entered through either channel 6 or 7 . A flow rate, uniform for all channels, was chosen to give about a 60 -min average steady-state residence time in the flow-cell. The top and bottom of the migration pattern were read on scales placed at $2_{-}, 10_{-}, 16-, 22_{-}$, and $28-\mathrm{cm}$ locations downstream in the $30-\mathrm{cm}$ chamber, and the average of these two readings was taken as the position of the band. The readings were made by an

*The results of work now in progress on three-dimensional migration patterns in the STAFLO flow-cell will permit higher precision analytical interpretations of such studies. 


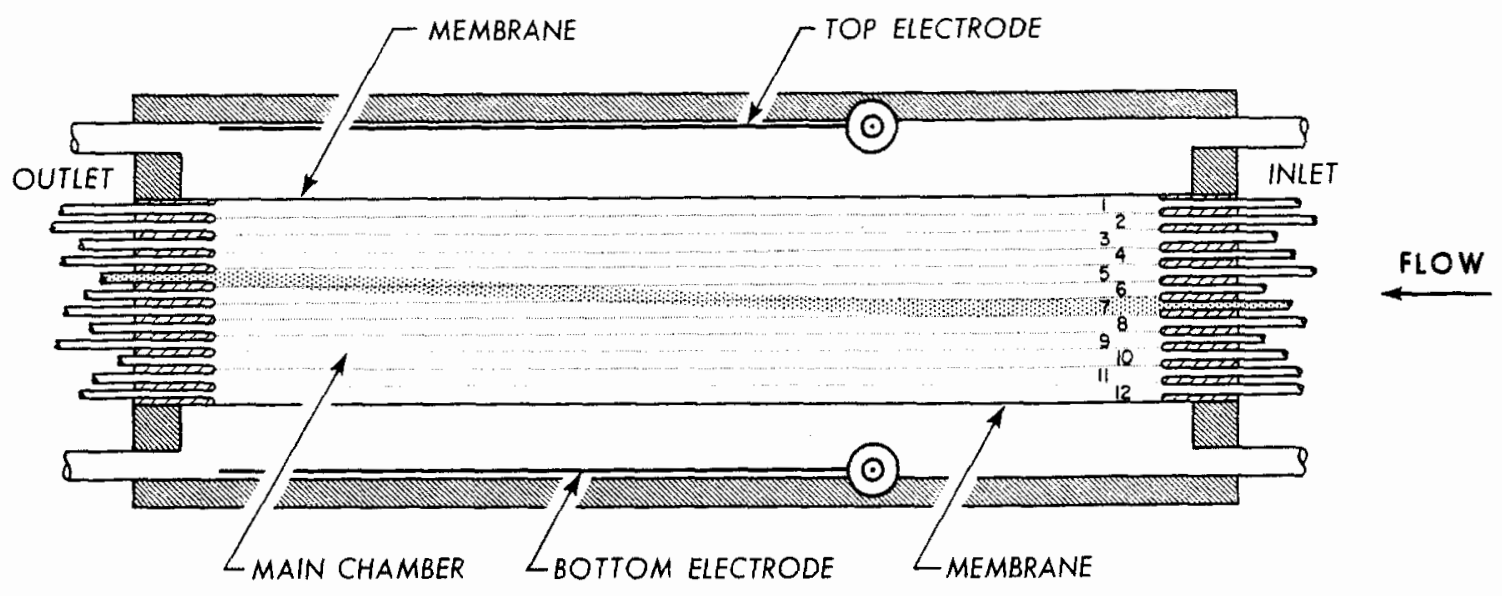

Figure 1. Schematic diagram of the STAFLO apparatus. MUB-6212

MIGRATION POSITIONS IN THE STAFLO FLOW-CELL

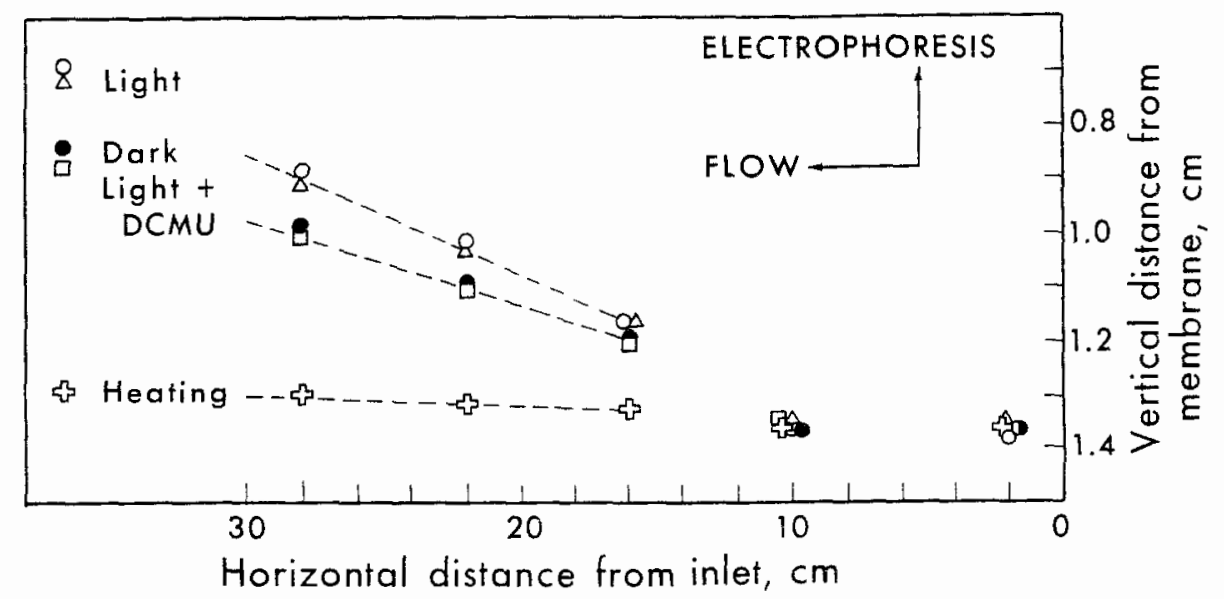

Figure 2. Migration positions in the STAFLO flow-cell. Chloroplasts were isolated and the experiment performed in $44 \mathrm{mM} \mathrm{NaCl}, 20 \mathrm{mM}$ tris-HCl (pHi 8.0). The chloroplasts suspension entered in channel 6 and the position of the migration pattern was determined as described in Materials and Methods (note the large expansion of the vertical scale). An electric field of $1.33 \mathrm{~V} / \mathrm{cm}$ begins at the $10 \mathrm{~cm}$ position and the positive electrode is up. The migration patterns correspond to the following conditions: Run 1, $O$, Iight; Run 2, $\square$, light plus $10 \mu \mathrm{M}$ DCMU; Run 3, $\Delta$. light; Run 4, 0 , dark; and Run 5, s; , heating and sedimentation control (see Materials and Methods). MUB-6955 
independent observer unfamiliar with the experiment being performed. Each of the 12 equal channels corresponds to a $0.25-\mathrm{cm}$ height in the main migration chamber. It was observed that the chloroplast suspension entered the flow-cell with the theoretical width of $0.25 \mathrm{~cm}$, left with a width of 0.24 to $0.27 \mathrm{~cm}$ and was very uniform throughout the passage along the migration chamber.

Conventional microelectrophoresis was performed with a rectangular NorthrupKunitz-type cell $(0.86 \mathrm{~mm}$ thick and $12 \mathrm{~mm}$ wide) mounted in the lateral position (5). A current of $3 \mathrm{~mA}$ was used. The symmetrical $\mathrm{Zn}-\mathrm{ZnSO}_{4}$ electrodes were isolated from the microelectrophoresis chamber by the following sequence of barriers: a fine sintered glass disk, a chamber filled with saturated $\mathrm{Na}_{2} \mathrm{SO}_{4}$ and a glass tube filled with the experimental medium (plus 10\% dextran to minimize convective exchange of specimen and buffer at the stopcock). The particle velocity at different depths in the chamber was determined as the number of divisions of the calibrated eyepiece reticule crossed by the biological particle per unit time, with the electric field first in one direction, then reversed. The stationary-level electrophoretic velocity was determined from the parabola obtained by a least squares computer fit of the measured points.

Except as noted, light was provided by two 150-watt reflector flood lamps filtered through $10 \mathrm{~cm}$ of water, giving an illumination of 70 foot candles. All experiments were run at $24^{\circ} \pm 1^{\circ} \mathrm{C}$. The resistivity of the various solutions was measured with a Leeds and Northrup conductivity cell and bridge (model 4960) and the pH was measured with a Beckman model G $\mathrm{pH}$ meter with a standard glass electrode and a calomel reference electrode.

EXPERIMENTAL MEDIA AND ELECTRICA:- PARAMETERS Chloroplasts were isolated and electrophoretically studied under three different conditions: $1.88 \mathrm{mM} \mathrm{NaCl}$, $25 \mathrm{mM}$ tris-HCI (pH 8.0); resistivity, $108 \mathrm{ohm-cm;} \mathrm{current,} 100 \mathrm{~mA}$ (i. e., current density of $7.3 \mathrm{~mA} / \mathrm{cm}^{2}$ ); abbreviated, $88: 25 ; 2.44 \mathrm{mM} \mathrm{NaCl}, 20 \mathrm{mM}$ tris $-\mathrm{HCl}$ ( $\mathrm{pH} 8.0$ ); resistivity, $182 \mathrm{ohm}-\mathrm{cm}$; current, $100 \mathrm{~mA}$; abbreviated $44: \overline{20} ; 3.175 \mathrm{mM} \mathrm{NaCl}, 50 \mathrm{mM}$ tris $-\mathrm{HCl}(\mathrm{pH} 8.0)$; resistivity, $60 \mathrm{ohm-cm}$; current, $300 \mathrm{~mA}$; abbreviated, $175: \overline{50}$.

\section{RESULTS}

STAFLO EXPERIMENTS IN MEDIUM 88:25 Chloroplast electrophoretic mobilities in the light and in the dark, as well as the light:dark mobility ratios, are given for five experiments in Table 1. In each case the mobility in the dark is smaller than the mobility in the light. The average mobility in the light is $-1.28 \mu / \mathrm{sec} / \mathrm{V} / \mathrm{cm}$, and in the dark is $-1.06 \mu / \mathrm{sec} /$ $\mathrm{V} / \mathrm{cm}$. This gives an average light:dark mobility ratio of $1.20 \pm 0.06$ (Standard Error of the Mean, SEM). Not unexpectedly, the spinach chloroplasts were found to be negatively charged, migrating toward the anode whether it was up or down. The light-induced mobility increase was $20-40 \%$ smaller after $7 \mathrm{hr}$ and was reversible, i. e., placing the sample in the light and then performing the electrophoresis in the dark gave the usual dark migration pattern and vice versa. To check on a possible nonspecific origin for this light effect, the migration pattern of human erythrocytes was observed in the light and in the dark. Erythrocytes are about 
Table 1. Electrophoretic mobility of spinach chloroplasts*

\begin{tabular}{cccc}
\hline${ }^{-\mu_{L}}$ & $\begin{array}{c}-\mu_{D} \\
(\mu / \mathrm{sec} / \mathrm{V} / \mathrm{cm})\end{array}$ & $\mu_{L} / \mu_{D}$ \\
1.03 & 0.99 & 1.04 \\
1.52 & 1.09 & 1.39 \\
1.32 & 1.03 & 1.28 \\
1.46 & 1.27 & 1.15 \\
$\mathrm{Av}$ & 1.06 & 0.93 & 1.14 \\
\hline
\end{tabular}

*Chloroplasts were prepared as described in Materials and Methods, and STAFLO-electrophoresis was performed in $88 \mathrm{mM} \mathrm{NaCl}, 25 \mathrm{mM}$ tris $-\mathrm{HCl}$ (pH 8.0) within four hours after isolation. The electrophoretic velocity was determined as the migration distance divided by the migration time. The electric field strength was calculated as the product of the current density $\left(0.0073 \mathrm{amps} / \mathrm{cm}^{2}\right.$ for the $19.7 \times 0.7 \mathrm{~cm}$ platinum strip electrode) and the resistivity $(108 \mathrm{ohm}-\mathrm{cm})$. The electrophoretic mobility, $\mu$, is the electrophoretic velocity divided by the field strength. L and D represent light and dark respectively. Errors indicated are Standard Error of the Mean. The deviation of the light:dark mobility ratio, $\mu_{L} / \mu_{D}$ (given in the last column), from 1.00 is a measure of the magnitude of the light-induced mobility increase.

the same size as chloroplasts and also contain a similar pigment. No light-dark difference in STAFLO migration pattern was detected for erythrocytes.

STAFLO EXPERIMENTS IN MEDIUM 44:20 With this higher resistivity medium, a higher field strength could be developed for the same current density. For fourteen experiments, the mobility in the light was $1.52 \pm 0.06(\mathrm{SEM}) \mu / \mathrm{sec} / \mathrm{V} / \mathrm{cm}$ and in the dark was $1.38 \pm 0.06$ (SEM), giving a light:dark mobility ratio of $1.11 \pm 0.02$ (SEM). As for the higher ionic strength experiments, the absolute values of the mobilities varied considerably from day to day with different preparations, yet the light:dark ratio was never less than 1.00. Data from typical migration patterns are shown in Fig. 2. The migration slope (i.e., electrophoretic velocity) in the light is seen to be greater than in the dark.

The possible involvement of the electron transport and energy transfer pathways was next investigated. For example, when $10 \mu \mathrm{M} 3-(3,4$ dichlorophenyl)-1, 1-dimethylurea (DCMU), an inhibitor of oxygen evolution, was present in the light, the pattern was indistinguishable from the dark pattern (Fig. 2). DCMU had no effect in the dark (Table 2). In two other experiments (Table 2), $100 \mu \mathrm{M}$ o-phenanthroline, another oxygen-evolution inhibitor, also seemed to abolish the light effect. Neither $5 \mathrm{mM} \mathrm{NH}_{4} \mathrm{Cl}$ nor $50 \mu \underline{M}$ quinacrine, uncouplers of photophosphorylation, changed the migration pattern in the light or dark. Phenazine methosulfate (PMS), a cyclic electron carrier, was added next. The resulting average light:dark mobility ratios were as follows: no addition, 1.14 \pm 0.04 ; with addition of $20 \mu \mathrm{M} P M S, 1.15 \pm 0.02$, 
Table 2. Biochemical modification of the light:dark mobility ratios *

\begin{tabular}{|c|c|c|c|c|}
\hline \multirow{2}{*}{$\begin{array}{l}\text { Experimental medium } \\
(\mathrm{mM} \mathrm{NaCl}: \mathrm{mM} \text { tris }-\mathrm{HCl} \text {, } \\
\text { pH 8.0) }\end{array}$} & \multirow[b]{2}{*}{ Substance added } & \multirow[b]{2}{*}{ Concentration } & \multicolumn{2}{|l|}{$\mu_{L} / \mu_{D}$} \\
\hline & & & No addition & Addition \\
\hline $88: 25$ & DCMU & $10 \mu \mathrm{M}$ & 1.39 & 1.00 \\
\hline $44: 20$ & $\mathrm{DCMU}$ & $10 \mu \mathrm{M}$ & 1.27 & 0.98 \\
\hline $44: 20$ & o-Phenanthroline & $100 \mu \mathrm{M}$ & 1.14 & 0.98 \\
\hline $44: 20$ & o-Phenanthroline & $100 \mu \underline{M}$ & 1.06 & 1.01 \\
\hline $175: 50$ & $\mathrm{NH}_{4} \mathrm{Cl}$ & $5 \mathrm{mM}$ & 1.10 & 1.12 \\
\hline $44: 20$ & $\mathrm{NH}_{4} \mathrm{Cl}$ & $5 \mathrm{~m} \bar{M}$ & 1.17 & 1.16 \\
\hline $175: 50$ & Quinacrine & $50 \mu \underline{M}$ & 1.49 & 1.42 \\
\hline $88: 25$ & Quinacrine & $50 \mu \underline{M}$ & 1.28 & 1.24 \\
\hline $44: 20$ & Phenazine methosulfate & $20 \mu \mathrm{M}$ & 1.07 & 1.14 \\
\hline $44: 20$ & Phenazine methosulfate & $20 \mu \bar{M}$ & 1.14 & 1.15 \\
\hline $44: 20$ & Phenazine methosulfate & $20 \mu \bar{M}$ & 1.09 & 1.11 \\
\hline $44: 20$ & Phenazine methosulfate & $20 \mu \underline{M}$ & 1.25 & 1.21 \\
\hline $44: 20$ & $\mathrm{HCl}$ & $\mathrm{pH} 7.1$ & 1.15 & 1.14 \\
\hline $44: 20$ & $\mathrm{HCl}$ & $\mathrm{pH} 7.1$ & 1.07 & 1.17 \\
\hline
\end{tabular}

*Chloroplasts were prepared as described in Materials and Methods, and STAFLO-electrophoresis was performed in $88 \mathrm{mM}$ NaCl, $25 \mathrm{mM}$ tris-HCl (pH 8.0) within four hours after isolation. The electrophoretic velocity was determined as the migration distance divided by the migration time. The electric field strength was calculated as the product of the current density $\left(0.0073 \mathrm{amps} / \mathrm{cm}^{2}\right.$ for the $19.7 \times-.7 \mathrm{~cm}$ platinum strip electrode) and the resistivity (108 ohm-cm). The electrophoretic mobility, $\mu$, is the electrophoretic velocity divided by the field strength. I and D represent light and dark respectively. Errors indicated are Standard Error of the Mean. The deviation of the light:dark mobility ratio, $\mu_{L} / \mu_{D}$ (given in the last column), from 1.00 is a measure of the magnitude of the light-induced mobility increase.

indicating that electron flow supported by PMS does not detectably affect the light-induced mobility increase. There also appeared to be no difference in the light: dark mobility ratio measured at $\mathrm{pH} 7.1$ compared with that at $\mathrm{pH} 8.0$ (both performed in tris-HC1).

Disruption of the chloroplasts by a $10-$ sec treatment in a Lourdes Nossal cell disintegrator abolished the light-induced mobility increase ( $T$ able 3), stressing the importance of membrane integrity for this effect. Since the 70 foot-candles employed in these experiments is a low-light intensity (most photosynthetic processes require much higher intensities for saturation), a light intensity of 300 foot-candles was tried in an attempt to increase the lightinduced change. No increase was noted (Table 3), although the increased heating from the higher light intensity made these results somewhat uncertain. However, the light-induced mobility increase seemed to be present at 5 foot-candles, indicating the extreme sensitivity of this charge change. 
Table 3. Physical modification of the light:dark mobility ratios*

Experimental medium

(mM NaCl:mM tris-HCl, pH 8.0)

\begin{tabular}{|c|c|c|c|c|}
\hline \multirow{2}{*}{$44: 20$} & \multirow{4}{*}{$\begin{array}{l}\text { Treated for } 10 \mathrm{sec} \text { in } \\
\text { a Lourdes Nossal shaker }\end{array}$} & \multicolumn{2}{|c|}{ Not treated } & Treate \\
\hline & & 1.09 & & 0.96 \\
\hline $44: 20$ & & 1.25 & & 1.00 \\
\hline \multirow[t]{3}{*}{$44: 20$} & & 1.12 & & 0.96 \\
\hline & & \multicolumn{3}{|c|}{ Light intensity, foot candles } \\
\hline & & 5 & 70 & 300 \\
\hline $44: 20$ & & & 1.06 & 1.04 \\
\hline $44: 20$ & & 1.10 & 1.09 & 1.03 \\
\hline $44: 20$ & & 1.00 & 1.03 & 1.00 \\
\hline $44: 20$ & & 1.06 & 1.11 & \\
\hline
\end{tabular}

*Chloroplasts were prepared as described in Materials and Methods, and STAFLO-electrophoresis was performed in $88 \mathrm{mM} \mathrm{NaCl}, 25 \mathrm{mM}$ tris-HCl ( $\mathrm{pH} \mathrm{8.0)}$ within four hours after isolation. The electrophoretic velocity was determined as the migration distance divided by the migration time. The electric field strength was calculated as the product of the current density $\left(0.0073 \mathrm{amps} / \mathrm{cm}^{2}\right.$ for the $19.7 \times 0.7 \mathrm{~cm}$ platinum strip electrode) and the resistivity $(108 \mathrm{ohm}-\mathrm{cm})$. The electrophoretic mobility, $\mu$, is the electrophoretic velocity divided by the field strength. L and $D$ represent light and dark respectively. Errors indicated are Standard Error of the Mean. The deviation of the light: dark mobility ratio, $\mu_{I} / \mu_{D}$ (given in the last column), from 1.00 is a measure of the magnitude of the light-induced mobility increase. The light intensity indicated was obtained by varying the number and position of 150 -watt reflector flood lamps.

Table 4. Effect of DCMU on microelectrophoretic mobilities *

\begin{tabular}{ccc}
$-\mu_{L}$ & $\left.-\mu_{(L+D C M U}\right)$ & $\mu_{L} / \mu_{(L+D C M U)}$ \\
$(\mu / \mathrm{sec} / \mathrm{V} / \mathrm{cm})$ & $(\mu / \mathrm{sec} / \mathrm{V} / \mathrm{cm})$ & \\
1.54 & 1.39 & 1.11 \\
1.25 & 1.05 & 1.19 \\
1.40 & 1.32 & 1.06 \\
1.50 & 1.28 & 1.17 \\
\hline
\end{tabular}

"Chloroplasts were isolated and the experiments performed in $88 \mathrm{mM} \mathrm{NaCl}, 25 \mathrm{mM} \mathrm{tris}-\mathrm{HCI}$ (pH 8.0). After a 22-min light incubation, the velocity measurements were performed in an iliuminated microelectrophoresis cell (measurement of an entire parabola took $10 \mathrm{~min}$ ). The subscripts to the mobilities $(\mu)$ refer to light (L) and to light plus $10 \mu M D C M U(L+D C M U)$. 
MICROELECTROPHORESIS EXPERIMENTS To check some of the above observations, microelectrophoresis was performed using chloroplasts isolated and measured in $88 \mathrm{mM} \mathrm{NaCl}, 25 \mathrm{mM}$ tris-HCl (pH 8.0). Since illumination is necessary to observe the chloroplasts in, the microscope, all of these experiments had to be performed in the light. The results, given in Table 4, showed that $10 \mu \mathrm{M}$ DCMU caused a lowering of the "light" mobility, in agreement with the findings by STAFLO electrophoresis. Five $\mathrm{mM} \mathrm{NH}_{4} \mathrm{Cl}$ did not cause a significant change in the "light" mobility. For eleven microelectrophoresis runs the mobility for chloroplasts kept $22 \mathrm{~min}$ in the light averaged $-1.36 \pm 0.04(\mathrm{SEM}) \mu / \mathrm{sec} / \mathrm{V} / \mathrm{cm}$.

\section{DISCUSSION}

Isolated spinach chloroplasts have been shown to carry a net negative charge by two different methods, in agreement with results for plant and animal mitochondria (6-9), for Nitella chloroplasts (10) and for fragments of spinach chloroplasts (11). In the light, the STAFLO-electrophoretic mobility, which presumably measures the net charge within the surface of shear, was found to be about 15\% larger than in the dark.

The electrophoretic mobility of the chloroplasts in the light, about $-1.3 \mu / \mathrm{sec} / \mathrm{V} / \mathrm{cm}$ [in $88 \mathrm{mM} \mathrm{NaCl}, 25 \mathrm{mM}$ tris-HCl (pH 8.0)], is smaller than the $-2.6 \mu / \mathrm{sec} / \mathrm{V} / \mathrm{cm}$ reported for Nitella chloroplasts [in buffers of ionic strength 0.01 (pH 7)] (10). It is slightly larger than the -0.5 to $-1.1 \mathrm{\mu} / \mathrm{sec} / \mathrm{V} / \mathrm{cm}$ range reported for mitochondria (in different buffers near pH 7) (6-9) and is similar to that for erythrocytes $(5,12,13)$, i. e., the electrophoretic mobility of chloroplasts does not appear unusual in either sign or magnitude. The microelectrophoretic mobility of spinach chloroplasts measured here varied more from experiment to experiment than did the microelectrophoretic mobility of human erythrocytes, possibly reflecting the observed greater heterogeneity in the chloroplast suspensions.

For the ionic strengths employed in this study, the usual equation of electrophoretic motion (for large spherical particles of low conductivity) can be applied (5):

$$
\text { electrophoretic mobility }=\frac{D \zeta}{4 \pi \eta} \text {, }
$$

where $D$ is the dielectric constant of the suspending medium, $\zeta$ is the potential difference between the surface of shear and the bulk of the liquid, and $\eta$ is the viscosity of the suspending medium. Using an electrophoretic mobility of $-1.3 \mu / \mathrm{sec} / \mathrm{V} / \mathrm{cm}$, the calculated zeta potential is $-17 \mathrm{mV}$ for spinach chloroplasts.

For spherical particles, the surface charge density, $\sigma$, is given by (12):

$$
\sigma=\sqrt{\frac{N D k T}{2,000 \pi}} \sqrt{\sum_{i} c_{i}\left(e^{-z_{i} e \zeta / k T}-1\right)}
$$

where $c_{i}$ and $z_{i}$ represent the molar concentration and charge, respectively, for the $i^{\text {th }}$ ion. For $88 \mathrm{mM} \mathrm{NaCl}, 25 \mathrm{mM}$ tris-HCl (pH 8.0), this gives $\sigma=-3.7 \times 10^{3} \mathrm{esu} / \mathrm{cm}^{2}$. Correcting for the finite size of ions in the double layer (12), this becomes $-2.7 \times 10^{3} \mathrm{esu} / \mathrm{cm}^{2}$ or $-5.6 \times 10^{12}$ electronic charges $/ \mathrm{cm}^{2}$. Hence, negative charges should be about $40 \AA$ apart, 
on the average, which is similar to the charge density for erythrocytes (12, 13). Using a chloroplast volume of $100 \mu^{3}(14)$ and assuming a spherical shape, the surface area is $1.03 \times 10^{-6} \mathrm{~cm}^{2}$, corresponding to $5.8 \times 10^{6}$ net negative charges per chloroplast. Based on the above equations, a 15\% light-induced mobility increase corresponds to a net change of $0.8 \times 10^{6}$ electronic charges per chloroplast amounting to a charge concentration of $0.013 \mu \mathrm{M}$ for $10^{7}$ chloroplasts $/ \mathrm{ml}$. The $15 \%$ mobility change associated with the relatively small charge change emphasizes the strength of electrical forces. Based on the number of chloroplasts/mg chlorophyll $\left(15 \times 10^{8}\right)$, one calculates $4.5 \times 10^{8}$ chlorophyll molecules per chloroplast or about 560 chlorophyll molecules per induced charge.

It is interesting to calculate the number of quanta absorbed per chloroplast under the electrophoresis conditions, in order to compare it with the charge change. A gas-filled tungsten lamp operates at about 20 lumens per electrical watt, which corresponds to $22 \mu w a t t s / \mathrm{cm}^{2}$ (or $6.7 \times 10^{13}$ quanta/ $\mathrm{sec}-\mathrm{cm}^{2}$ ) in the interval 595 to $605 \mathrm{m \mu}$, for 70 foot-candles intensity (15). Numerical integration of the spectral distribution curve from 400 to $700 \mathrm{~m} \mu$ gave $1.50 \times 10^{15}$ quanta/sec-cm in the visible range. Using specific absorption coefficients for chlorophyll (16) and assuming Beer's law, $8.8 \%$ of this light was calculated to be absorbed for a suspension of $10^{7}$ chloroplasts $/ \mathrm{ml}$. Hence $1.9 \times 10^{7}$ quanta are absorbed per chloroplast for 70 footcandies. If each electronic unit of charge were caused by one absorbed quantum, then the $0.8 \times 10^{6}$ increased charges per chloroplast could be produced in 0.04 sec. That is, the charge change leading to the light-induced mobility increase would not decay in less than about 0.04 sec. To compare the light absorption with the oxygen evolution under electrophoresis conditions, a Clark Oxygen Electrode was employed and an electron acceptor was added. Under these conditions with $1 \mathrm{mM}$ ferricyanide present, the oxygen evolution in the light was $0.6 \mu \mathrm{M} /$ min, corresponding to $25 \%$ efficiency in chloroplast utilization of the absorbed light (assuming 8 quanta are necessary per oxygen molecule evolved).

Since STAFLO electrophoresis enables one to measure the electrophoretic velocity in the dark as well as in the light, the effect of light on the charge state of the chloroplast could be investigated directly. The distance migrated in the electric field per unit time was greater in the light than in the dark, an effect inhibited by DCMU. It was possible to confirm the DCMU effect by microelectrophoresis as DCMU caused a $13 \%$ decrease of the mobility measured in the light. This is suggestive of some kind of metabolic involvement in the light-induced changes.

The apparent lack of effect of $\mathrm{NH}_{4} \mathrm{Cl}$, quinacrine, $\mathrm{pH}$, and PMS on the light-induced mobility increase suggests that a high-energy intermediate of photophosphorylation is probably not involved in the mobility changes. A high-energy intermediate has been implicated in the uptake of various ions by spinach chloroplasts (17) and in the light-induced $\mathrm{pH}$ rise in unbuffered suspensions of chloroplasts (18). Hence the transport of ions mediated by the energy transfer pathway appears to be an unlikely explanation for the difference in electrophoretic mobility observed in the light and dark. Altered hydrogen ion transport associated with the photo-evolution of oxygen might, however, be involved. Some oxygen evolution by chloroplasts has been shown to occur in the absence of an added electron acceptor and this process 
can be inhibited by DCMU (19). Hence, the inhibition by DCMU of the light-induced mobility increase may be due to a binding of DCMU at some site involved with the early steps of oxygen evolution, thereby preventing charge separation.

From a physical standpoint, changes in ion transport could lead to altered net charge characteristics, including induction of an internal net charge. Although electrophoretic attempts to detect such charge effects in other cellular species have been largely unsuccessful (13), the question should not be considered closed for an energy-transducing species such as a chloroplast. A recent suggestion has been advanced for the existence of an internal zeta potential in experimentally altered nerve (20). In addition to net charge effects, altered transport (or membrane permeability) could also alter mobility by changing internal chloroplast conductivity, but according to electrophoretic theory this would require a light-induced decrease in conductivity. A variation in ions absorbed on the chloroplast surface could also lead to a mobility change.

Changes in volume or shape could lead in several ways to a mobility increase. If the membrane were distorted (unfolding, stretching, exposing or concealing charged groups), such changes might increase surface charge density or otherwise alter the structure or dynamics of the electrical double layer, e.g., by surface conductance, electrosmotic or streaming potential effects. If these changes resulted in an increased zeta potential, we would expect an increased electrophoretic mobility [Eq. (1)]. A light-dependent swelling has, in fact, been observed for chloroplasts (14). To compare swelling with the light-induced mobility increase, experiments were performed under identical conditions to those used in the electrophoresis studies (21). As for the mobility change, the light-induced swelling (measured by the decrease in optical density at $540 \mathrm{m \mu}$ ) is not detectably affected by $\mathrm{NH}_{4} \mathrm{Cl}$, quinacrine, or pH changes. In contrast to the electrophoresis results, however, DCMU, o-phenanthroline, and Nossal treatment are without effect on the light-induced swelling, while PMS clearly stimulates it. Furthermore, even in the dark, chloroplast volume increases continuously with time, and both the dark and light swelling is irreversible (21). Thus, swelling does not appear to be the principal cause of the mobility increase. It was argued above that swelling could alter surface structure, and consequently affect the mobility, but it can also be argued that swelling need not do so. A gradual transformation from a less spherical to a more spherical shape could occur without changing the stress on the membrane or the small-scale surface structure, as is apparently the case for red blood cells (22).

A light-induced shrinkage has been well characterized for spinach chloroplasts (see references in 17, 21) and should be considered as a possible basis for the charge change. It appears unlikely to be the principal cause, however, as shrinkage is greatly enhanced by PMS, is abolished by uncouplers of photophosphorylation, and has not been observed under the conditions employed for electrophoresis.

Finally, a coordinated solid-state-type energy transfer mechanism might possibly be involved in moving the charge out from its internal site of photo-production to the chloroplast outer surface. Attention has been called to the possible semiconductor properties of local 
regions of photosynthetic structures (23). This mechanism might require integrity of the membrane, which would explain why mechanical disruption of the chloroplasts could abolish the light-induced mobility increase. Furthermore, the apparent ability of a low-light intensity to give rise to the observed effect is not inconsistent with this conduction hypothesis. Important for distinguishing between various mechanisms will be more detailed knowledge of the time constant for development of the mobility increase.

In conclusion, it might be noted that a number of cross-coupled transduction phenomena are now known for chloroplasts, including light-chemical, light-mechanical (shape, volume changes), chemical-mechanical, and the present light-electrical effects. Among the intriguing possibilities for future study are the other cross-coupled phenomena such as electrical-mechanical, chemical-electrical, etc., as well as higher-order interaction phenomena. Such investigations should aid us in understanding the multifaceted character of the semipermeable, flexible, viscoelastic $(24,25)$ structures that are biological membranes.

\section{SUMMARY}

Stable-flow free-boundary electrophoresis of isolated spinach chloroplasts was performed in various buffered saline solutions, in the light as well as in the dark. The chloroplasts were negatively charged, and the absolute value of their electrophoretic mobility was about 15\% larger in the light than in the dark. This light-induced mobility increase could be abolished by $3-(3,4$ dichlorophenyl)-1, 1-dimethylurea, a finding confirmed by conventional microelectrophoresis. The increase appeared to be unaffected by uncouplers of photophosphorylation or by a cyclic electron carrier, in distinction to light-induced shrinkage or ion translocation. Contrary to light-induced swelling, the mobility increase was observed under low-light intensity and was lost upon mechanical disruption of the chloroplasts. A physical rather than metabolic mechanism appears most consistent with all the observations.

\section{ACKNOWLEDGMENTS}

We wish to thank Gretchen Hylton for her assistance, including reading of the STAFLO migration patterns, and Tom Tenforde for the microelectrophoretic measurements and data reduction. This work was supported in part by the United States Atomic Energy Commission and Lawrence Radiation Laboratory, and by the National Science Foundation grant (GB-1550) of Dr. Lester Packer, whose continued interest and helpful discussions are gratefully acknowledged. One of us (P.S. N.) has been supported by a National Science Foundation Predoctoral Fellowship.

\section{REFERENCES AND NOTES}

1. Nishizaki, Y.; Plant Cell. Physiol. 4: 353, 1963.

2. Nagai, R., and Tazawa, M. ; Plant Cell. Physiol. 3: 323, 1962.

3. Whatley, F. R., and Arnon, D. I.; in Methods in Enzymology VI, edited by S. P. Colowick and N. O. Kaplan, New York, Academic Press, 1963.

4. Mel, H. C.; J. Theor. Biol. 6:307, 1964.

5. Brinton, C. C., and Lauffer, M. A.; in Electrophoresis, edited by M. Bier, New York, Academic Press, 1959. 
6. Estermann, E. F.; Conn, E. E., and McLaren, A. D.; Arch. Biochem. Biophys. 85: 103, 1959.

7. Thompson, T. E., and McLees, B. D.; Biochim. Biophys. Acta 50: 213, 1961.

8. Douglas, H. W.; Laycock, M. V., and Boulter, D.; J. Exptl. Bot. 14: 198, 1963.

9. Davenport, J. B.; Biochim. Biophys. Acta 88: 177, 1964.

10. Mercer, F. V.; Hodge, A. J.; Hope, A. B., and McLean, J. D.; Aust. J. Biol. Sci. 8: 1, 1955.

11. Gross, J. A.; Becker, M. J., and Shefner, A. M. ; Experientia 20:261, 1964.

12. Abramson, H. A.; Moyer, L. S., and Gorin, M. H. ; Electrophoresis of Proteins, New York, Reinhold Publishing Corp., 1942.

13. Glaeser, R. M.; Ph. D. Thesis, University of California, Lawrence Radiation Laboratory Report, UCRL-10898, 1963.

14. Packer, L.; Siegenthaler, P. A., and Nobel, P. S.; J. Cell. Biol., in press.

15. IES Lighting Handbook, 3rd Ed, Illuminating Engineering Society, New York, 1959.

16. French, C. S.; in Encyclopedia of Plant Physiology Vol. 5, edited by W. Ruhland, Berlin, Springer-Verlag, 1960.

17. Nobel, P. S., and Packer, L.; Plant Physiol., in press.

18. Neumann, J., and Jagendorf, A. T.; Arch. Biochem. Biophys. 107: 109, 1964.

19. Fork, D. C.; Plant Physiol. 38: 323, 1963.

20. Chandler, W. K.; Hodgkin, A. L., and Meves, H.; J. Physiol. 1965, in press.

21. Nobel, P. S.; Ph. D. Thesis, University of California, Berkeley, 1965.

22. Rand, R. P., and Burton, A. C.; Biophys. J. 4: 115, 1964.

23. Calvin, M.; Brookhaven Symp. in Biol. 11: 160, 1958.

24. Rand, R. P.; Biophys. J. 4: 303, 1964.

25. Katchalsky, A.; Kedem, O.; Klibansky, C., and De Vries, A.; in Flow Properties of Blood and Other Biological Systems, edited by A. L. Copley and G. Stainsby, New York, Pergamon Press, 1960, pp. 155-171.

This work was presented in part at the Ninth Annual Meeting of the Biophysical Society, San Francisco, Calif., February 24-26, 1965.

The data in this study were taken from a dissertation submitted to the Graduate School, University of California, Berkeley, by Park S. Nobel in partial fulfillment of the requirement for the Ph. D. degree.

Received August, 1965. 


\title{
Collection of Erythropoietin from Urine of Patients with Anemia Secondary to Hookworm
}

\author{
Abraham Gutnisky, Mary Lou Nohr, Luis Malgor and Donald Van Dyke
}

More abundant and less costly sources of erythropoietic hormone are needed (1). Erythropoietin can be concentrated from the plasma or urine of acutely hypoxic or severely anemic animals or human beings $(2-6)$, the least expensive method of preparation using urine of severely anemic patients (7-9). Very high concentrations of erythropoietin are found in the urine of some patients with severe hypoplastic anemia, hemolytic anemia, blood loss anemia or anemia secondary to leukemia. However, the urine of many such patients must be assayed in order to find that urine which has a sufficiently high concentration of erythropoietin to be valuable as a source, and the urines with the highest concentrations are often from patients who are terminal and very few in numbers. This study of the erythropoietin extract of a large number of patients with severe anemia secondary to hookworm was undertaken in an attempt to find a more reliable and inexpensive source of human urinary erythropoietin.

\section{MATERIALS AND METHODS}

The people of the province of Corrientes in northern Argentina are severely infected with Necator americanus (10). The capital city, Corrientes, serves a large rural population and its hospitals continually receive patients with hematocrits below $15 \%$ and hemoglobin below $5 \mathrm{~g} / 100 \mathrm{ml}$. Two of the main hospitals were provided with freezers and glass bottles and the medical staff were requested to freeze immediately all urine on voiding of patients with hemoglobin under $5 \mathrm{~g}$. Collection of urine was continued until the patient's hemoglobin rose to $5 \mathrm{~g}$ or more as a result of therapy. The frozen urine was transported daily to a large storage freezer at the erythropoietin collection center in the Department of Physiology of the School of Medicine, Northeast University, Corrientes.

The urine was thawed, an aliquot removed for assay as unmodified urine, and the remainder processed by the collodion adsorption method (11), the yield from each patient being assayed separately. All samples were assayed by the starved rat assay (12), using rats of the Wistar strain. Extracts with similar erythropoietic activity were combined to form large batches and re-assayed by the starved rat assay and by the ex-hypoxic mouse assay (13).

\section{RESULTS}

The results from 60 days' collection are reported in this paper. During that time the urine of 17 patients was collected, processed, and assayed. Of these, 13 had severe anemia secondary to hookworm, one had acute hemorrhage from a duodenal ulcer, one had aplastic anemia, one hemolytic anemia and one deGugliemo's syndrome. Of the 17 patients processed, only the one with aplastic anemia and the one with hemolytic anemia had insufficient 
Table 1. Erythropoietin from urine of severely anemic patients in Corrientes, Argentina

\begin{tabular}{|c|c|c|c|c|c|}
\hline Diagnosis & $\underset{\%}{\mathrm{HCT}}$ & $\begin{array}{l}\mathrm{Hb} \\
\mathrm{g} / 100 \mathrm{ml}\end{array}$ & $\begin{array}{c}\text { Total soluble } \\
\mathrm{mg}\end{array}$ & $\%$ recovery & $\begin{array}{l}\text { Activity } \\
\text { per } \mathrm{mg}\end{array}$ \\
\hline Hookworm & 7.5 & 1.9 & 156 & & 7.4 \\
\hline Hookworm & 16 & 3.2 & 259 & & 14.0 \\
\hline Hookworm & 11 & 2.5 & 586 & & 4.5 \\
\hline Hookworm & 14 & 2.8 & 679 & & 15.6 \\
\hline Hookworm & 12 & 2.4 & 799 & & 15.6 \\
\hline Hookworm & 17 & 3.7 & 877 & & 15.6 \\
\hline Hookworm & $\begin{array}{l}20 \\
15 \\
14\end{array}$ & $\begin{array}{l}4.3 \\
3.7 \\
3.1\end{array}$ & $648^{* *}$ & & 5.6 \\
\hline Hookworm & 14 & 4.3 & 448 & 29 & 24.5 \\
\hline Hookworm & 11 & $\begin{array}{l}2.7 \\
5.0\end{array}$ & $\begin{array}{l}438 \\
449\end{array}$ & 58 & $\begin{array}{r}33.3 \\
5.2\end{array}$ \\
\hline Hookworm & 15 & 3.3 & 388 & 37 & 4.4 \\
\hline Hookworm & 14 & 3.3 & 752 & 37 & 6.1 \\
\hline deGugliemo's & 11 & 4.4 & 574 & 41 & 9.1 \\
\hline Aplastic & 24 & 4.3 & 1,370 & 14 & 1.0 \\
\hline Acute blood loss & 15 & 3.7 & 135 & & 28.0 \\
\hline Hemolytic & 9 & 3.1 & 1,952 & & 2.8 \\
\hline
\end{tabular}

*Combined extract from three patients.

erythropoietin to be useful, Table 1. In the first part of the study, the unmodified urine as well as the extract were assayed in order to determine efficiency of extraction by the collodion adsorption method, Table 1. As can be seen from the table, recovery varies from $14 \%$ to $58 \%$ and activity of the extract from the urine of patients with hookworm ranged from 4.4 to 33 units per $\mathrm{mg}$ of $\mathrm{dry}$, soluble powder. In the 60-day period, one technician working full time

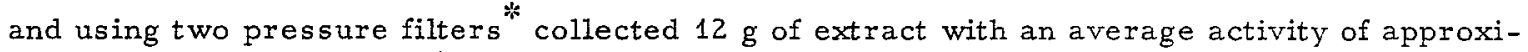
mately 9 standard B units ${ }^{\dagger}$ per milligram, or a total of 108,000 standard B units.

\section{DISCUSSION}

This and a previous study (14) demonstrate that the urine from patients with hemoglobin below $5 \mathrm{~g}$ caused by hookworm infection very frequently contains a high concentration of erythropoietin. By collecting in a heavily infested area such as Corrientes, Argentina, one can obtain an abundant supply of such urine. The present experience indicates that if one limits processing to patients with hookworm and hemoglobin below $5 \mathrm{~g}$, preliminary assay of the urine and even assay of the individual extracts may not be necessary unless one wishes to

*F. R. Hormann and Company, Inc., Model 9.

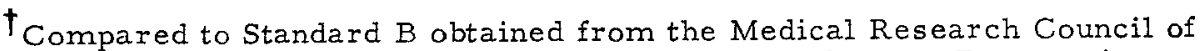
Great Britain, recently established as the International Reference Preparation. 
separate the very active from the moderately active. By eliminating the need for assay of the extract from each donor, one is able to reduce the cost of preparation very greatly. If methods can be developed which provide more quantitative recovery, the cost of producing erythropoietin could be further reduced, as recovery by the method used averaged less than $50 \%$.

It is estimated (15) that one-fourth of the world's population is infected with ancylostoma, particularly in warm, humid regions, although how many are severely anemic is not known, and it is not likely that this age-old scourge of mankind will be eliminated in the near future. In these studies it was found that the establishment of an erythropoietin collection center has created interest in and focused attention on the severity of the problem in the community and has contributed to improved patient care.

\section{SUMMARY}

More abundant and less costly sources of erythropoietic hormone are needed. It has been found that the urine of patients with severe anemia (less than $5 \mathrm{~g}$ hemoglobin) caused by hookworm consistently contains high concentrations of erythropoietin. The specific activity of the material collected was from 4.4 to 33.3 units per milligram with an average activity of 9 units/mg. It is believed that this is the least costly, most reliable, and most abundant source of erythropoietin available at present.

\section{ACKNOWLEDGMENTS}

The authors are indebted to Dr. Geoffrey Keighley of the California Institute of Technology for the ex-hypoxic mouse assays.

This work was carried out at Facultad de Medicina, Universidad Nacional del Nordeste, Corrientes, Argentina, with assistance by scientific personnel from Donner Laboratory of Medical Physics, University of California, Berkeley, California, and funded in part by the Consejo Nacional de Investigaciones y Tecnicas of Argentina (Grant No. 1991) and by the United States Atomic Energy Commission.

\section{REFERENCES}

1. Erythropoiesis, edited by L. O. Jacobson and M. Doyle, New York and London, Grune and Stratton, 1962.

2. Borsook, H.; Graybiel, A.; Keighley, G., and Windsor, E.; Blood 9: 734, 1954.

3. Hodgsori, G., and Toha, J.; Blood 9: 299, 1954.

4. Piliero, S. J.; Medici, P. T.; Pansky, B.; Luhby, A. I., and Gordon, A. S.; Proc. Soc. Expt1. Biol. Med. 93: 302, 1956.

5. Van Dyke, D. C.; Garcia, J. F., and Lawrence, J. H.; Proc. Soc. Expt1. Biol. Med. 96: $541,1957$.

6. Gurney, C. W.; Goldwasser, E., and Pan, C.; J. Lab. C1in. Med. 50: 534, 1957.

7. Van Dyke, D., and Pollycove, M. ; in Erythropoiesis, edited by L. O. Jacobson and M. Doyle, New York and London, Grune and Stratton, 1962, p. 340.

8. Stohlman, F., Jr.; New Eng1. J. Med. 267: 342, 392, 1962.

9. Lowy, P. H., and Borsook, H.; in Erythropoiesis, edited by L. O. Jacobson and M. Doyle, New York and London, Grune and Stratton, 1962, p. 33. 
10. Roche, M., and Layrisse, M.; Nature and causes of hookworm anemia. Monograph, in press.

11. Van Dyke, D. C.; Ciba Found. Symp. on Haemopoiesis, London, Churchill, 1960, p. 397.

12. Fried, W.; Plzak, L. F.; Jacobson, L. O., and Goldwasser, E.; Proc. Soc. Exptl. Biol. Med. 94: 237, 1957.

13. Lowy, P. H., and Keighley, G. L.; Clin. Chem. Acta., in press.

14. Van Dyke, D. C.; Layrisse, M.; Lawrence, J. H.; Garcia, J. F., and Pollycove, M.; Blood 18: 187, 1961.

15. CCTA/WHO (Commission for Technical Cooperation in Africa South of the Sahara), African Conference on Ancylostomiasis, Wld. Hlth. Org. Techn. Rep. Ser. 255, 1963.

Received November, 1965. 


\title{
ERP Studies of OH Radical in Ice and Several Crystal Hydrates
}

\author{
Thomas E. Gunter
}

Siegel et al. in 1960 (1) published the first of a series of papers (2-4), on studies of free radicals in the irradiated ice system, in which some evidence was given for identifying the radical producing part of the ESR spectrum, found in this system after irradiation at low temperature, as $\mathrm{OH}$ radical.

OH, which is known from microwave spectroscopy to be in a ${ }^{2} \pi_{3 / 2}$ ground state (5), would be expected, when hydrogen-bonded in a solid matrix, to show spin only paramagnetism. This means that if one takes the form of the Zeeman interaction to be

$$
\underline{\mathrm{H}}_{\mathrm{z}}=\beta(\overrightarrow{\mathrm{L}}+2 \overrightarrow{\mathrm{S}}) \cdot \overrightarrow{\mathrm{H}}
$$

the $L$ term contributes little to the observed paramagnetism. The cause of this in the case of molecular radicals is a strong bond that is overlap of molecular wave functions locking the molecule to the lattice. This molecular overlap takes the place of the strong crystal fields that produce spin only paramagnetism in iron group compounds. Strong bonding of the paramagnetic center to the lattice, through a noncentral interaction, implies nonconservation of orbital angular momentum; hence the average values of the components of $L$ are often zero. Therefore $L$, which is not a constant of the motion, is not an effective operator in the Hamiltonian given above.

The hyperfine interaction of an $S=1 / 2, I=1 / 2$ system such as $O H$ hydrogen bonded in a solid lattice would be expected to split each of the Zeeman levels into two levels, giving a total of four low-lying energy levels. Transitions such as $|\Delta M|=1,|\Delta m|=0$ are allowed by operators of the form $S_{ \pm}$, the electron spin raising and lowering operators, which are obtained from the time dependent Zeeman interaction between the spin system and the microwave magnetic field. Hence an equal intensity doublet is the expected ESR spectrum for $O H$ radical hydrogen bonded in a solid.

The radical $O D$ when trapped in a solid by hydrogen bonding should, like $O H$, show an effective electron spin of 1/2. Since the deuteron nuclear spin is 1, the OD ESR spectrum should be an equal line intensity triplet.

The evidence presented by Siegel et al. (1), for identifying the radical producing the high-field part of the spectrum shown in Fig. 1a as $O H$ and the corresponding high field of Fig. $1 \mathrm{~b}$ as $O D$, is that if one interprets the high field parts of these spectra as doublet and 


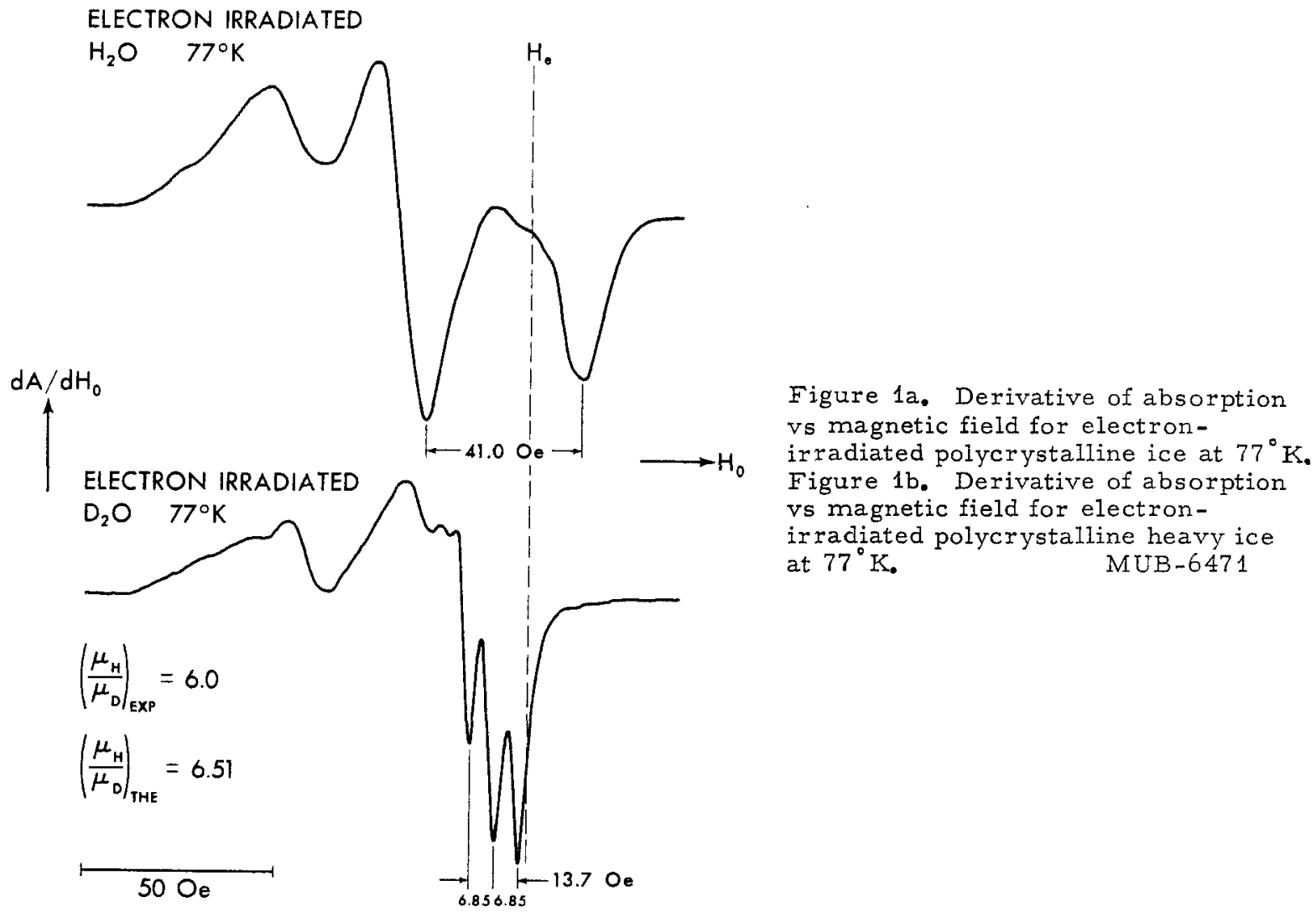

triplet respectively, then the measured splittings between maximum derivative points of the doublet and triplet spectra have a ratio near that of the proton to deuteron moments. In the case of our own measurements this ratio, as is seen in Fig. 1, is 41.0/6.85 or 6.0. The ratio of proton to deuteron moments is 6.5. Some considerations, however, suggest that more complete data should be presented before identifying the radical causing the irradiated $\mathrm{H}_{2} \mathrm{O} E S R$ lines as produced by $\mathrm{OH}$ radical.

If the interpretation as doublet and triplet respectively is correct, the conclusions that can be drawn from the irradiated $\mathrm{H}_{2} \mathrm{O}$ and irradiated $\mathrm{D}_{2} \mathrm{O}$ polycrystalline ESR spectra shown in Fig. 1 are that the radicals causing the spectra have an effective electron spin of $1 / 2$ and that they interact with a single proton or deuteron respectively. Other possibilities, then, for the radicals causing these spectra are $\mathrm{HO}_{2}$ and $\mathrm{DO}_{2}$.

As discussed above, $O H$ and $O D$ would be expected to produce an ESR doublet and triplet respectively, with lines of equal intensity. As can be seen from Fig. 1, this is far from the case for the lines observed in irradiated polycrystalline ice at $77^{\circ} \mathrm{K}$. Although arguments may be made for unequal line intensity being caused by anisotropy of line position with respect to field, more information is needed if such arguments are to carry any weight. 
McMillan et al. (6) have reported a study of irradiated single crystal ice. They assign a doublet with $\mathrm{g}_{\|}=2.0127, \mathrm{~g}_{1}=2.0077$, a (the isotropic or contact hyperfine interaction)= $41.3 \mathrm{Oe}, \mathrm{B}_{\|}=12 \mathrm{Oe}$, and $\mathrm{B}_{\perp}=-6$ Oe to the $\mathrm{OH}$ radical. The $\mathrm{B}^{\mathrm{s}} \mathrm{s}$ are the parallel and perpendicular parts of the dipole-dipole interaction between electron and proton. With a single crystal, this effective spin Hamiltonian would predict a high field line almost stationary in magnetic field position upon crystal rotation. The low field line is predicted to be 53 Oe from the high field line in the parallel orientation and 35 Oe from it in the perpendicular orientation.

When spectral lines are anisotropic in magnetic field upon crystal rotation, polycrystalline spectra of the same materials can show structure that gives greater weight to the perpendicular orientation than to the parallel orientation. This is true for the case of axial symmetry. The reason is, of course, that the probability of finding a spin at an angle $\theta$ with respect to the magnetic field goes like $\sin \theta$ for a random distribution of orientations. $\theta$ is the angle between the axis of the axially symmetric spin system and the external magnetic field. Hence, in polycrystalline ice the effective spin Hamiltonian given by McMillan et al. would show a strong high field line and a weak, broad low field line. This is the exact reverse of what is seen.

The contact interaction found by McMillan and co-workers is in poor agreement with that determined for $\mathrm{OH}$ in the vapor phase by Radford. Radford's results give a contact hyperfine splitting of $26.7 \mathrm{Oe}$. This work will be discussed in more detail later.

From their spectral data, McMillan et al. assign the radical axes to the directions from the center toward each of the corners of the hexagon of the ice lattice. That is, they are all taken to lie in the plane perpendicular to the optic axis of the ice crystal. This might be expected if ice had simple hexagonal symmetry. However, the ice space group is $\mathrm{D}_{6 \mathrm{~h}}^{4}-\mathrm{C} 6 / \mathrm{mmm}(7,8)$ the radical axes given by McMillan et al. make an angle of $90^{\circ}$ with one of the original hydrogen bond directions and $19^{\circ}$ with one of the other six original hydrogen bond directions. Intuitively it would appear more likely for $O H$ in ice to have its axis along one of the original hydrogen bond directions. This would be expected because in these orientations the radical would be held in position by four hydrogen bonds. There are seven hydrogen bond directions in the ice lattice. One of them, that along the optic axis, occurs twice as often as any of the other six.

Because of the above considerations, further work on positive identification of the ESR spectrum of $O H$ free radical in ice and on determination of the effective spin Hamiltonian parameters for this radical in the ice lattice was felt to be justified.

\section{EXPERIMENTAL TECHNIQUES}

ESR studies on irradiated $\mathrm{H}_{2} \mathrm{O}$ were carried out on irradiated ice samples in both polycrystalline and single crystal form. Irradiated $\mathrm{H}_{2} \mathrm{O}$ in the form of water of hydration in several crystal hydrates, were also studied. Single crystals of ice were grown by two techniques. The most satisfactory technique was found to be that discussed by Jona and Scherrer (9). The successful growing of single crystals of ice hinges on a few important factors: 
1. The optic axis of the ice crystal forms along the thermal gradient. Hence it is important to grow the crystals in a growing solution, in which the thermal gradient is uniform in direction over a volume large with respect to the desired volume of the ice crystals.

2. The temperature of the growing solution should be kept within approximately $1^{\circ} \mathrm{C}$ of the freezing point. The crystals should be grown slowly at a maximum rate of growth of approximately $11 / 2 \mathrm{~cm}$ of thickness per day.

3. Since ice supercools easily, the growing solution must be seeded.

The ice crystals grown for the experiments discussed in this paper were determined to be single crystals by studies with polarized light, by slow controlled melting techniques and by observation of the symmetries of the resulting ESR spectra after irradiation.

Single crystal ice samples were cut with a warm spatula in the shape of cylinders approximately $3 \mathrm{~mm}$ in diameter and up to $25 \mathrm{~mm}$ in length. The cylindrical axis was selected in the actual experiments to allow study of the ESR spectra for irradiated ice for rotation of the crystal with respect to the external magnetic field about orthogonal crystal axes.

The hydrated crystals studied, with the exception of the very slightly soluble $\mathrm{CaSO}_{4} \cdot 2 \mathrm{H}_{2} \mathrm{O}$, were grown by evaporation of the water or heavy water solvent from saturated solutions. Natural gypsum crystals free from paramagnetic impurities to about $5 \times 10^{-8}$ molar were used in the experiments on this sample.

Irradiation of the samples was by $6-\mathrm{MeV}$ electrons, except for one series of experiments on single crystal ice where the samples were irradiated with $250-k e V \mathrm{X}$ rays. The EPR spectra observed in single crystal ice after $X$ irradiation were similar to those observed in this type of sample after electron irradiation; hence these experiments will not be discussed separately from those using electron irradiation. The source of the electron beam was the Electron Linear Accelerator at the Lawrence Radiation Laboratory.

The samples were irradiated while submerged in liquid nitrogen. During irradiation the samples were usually held in a stainless steel sample holder mounted on the top of a double wall irradiation dewar. This sample holder held the sample near the lower tip of the dewar during irradiation and was used to transfer quickly the sample to a liquid nitrogen filled spectrometer dewar soon after irradiation. The sample holder allowed positioning of the sample within the microwave cavity and rotation of the sample with respect to the external magnetic field during EPR detection.

The EPR spectrometer used was of a typical field modulation type using $\mathrm{X}$ band microwave frequencies, a microwave bridge and reflection cavity, and a $100 \mathrm{kc} / \mathrm{sec} \mathrm{lock-in}$ detector. Hence the spectra were obtained in terms of derivative of absorption with respect to magnetic field vs magnetic field. An electronic integrator was sometimes used to plot directly absorption vs magnetic field. This was particularly useful for measurements of radiation yield of the radicals studied. The radiation yield of the radicals in irradiated ice was measured by comparing the area under the resonance absorption curve of the sample 
with that of a standard of known spin density. The standards used were DPPH and phosphorusdoped silicon in a standard calibrated by E. A. Gere of Bell Labs (10). Care was taken in these experiments to avoid microwave saturation of the resonance signals, to avoid dielectric loading of the microwave cavity (by use of small samples and careful placing of the samples within the TE 102 rectangular cavity) and to place both sample and standard at the same position in the cavity during measurement of their respective EPR signal.

\section{RESULTS AND DISCUSSION}

After preliminary experiments on irradiated ice and heavy ice in the polycrystalline form, Gunter and Jeffries (11) carried out a study of irradiated single crystal ice. The observed spectra consistently showed the necessary rotation symmetries ffor example a $60^{\circ}$ rotation symmetry was observed for spectra taken with the H field perpendicular to the optic axis) and were consistent even to small detail between samples.

The observed spectra, as shown in Fig. 2, were characterized by poor line resolution. Eight different crystal samples, in which rotation of the crystal was about the optic axis, were studied in order to verify that lack of good spectral resolution was not due to im. perfect or twinned crystals. In order to determine unambiguously the effective spin Hamiltonian of the radical or radicals producing the irradiated $\mathrm{H}_{2} \mathrm{O} E \mathrm{PR}$ spectra, it was thought useful to study irradiated $\mathrm{H}_{2} \mathrm{O}$ in the form of waters of hydration in simple hydrated crystals. In those hydrated crystals with a small number of sites for waters of hydration, it was hoped that a small number of radical sites would also be found.

Studies have now been carried out on several systems containing waters of hydration. Crystals that contain a large number of inequivalent water sites, such as $\mathrm{MgSO}_{4} \cdot 7 \mathrm{H}_{2} \mathrm{O}$ and $\mathrm{CdSO}_{4} \cdot 6 \mathrm{H}_{2} \mathrm{O}$, show the same type of spectral complexity as seen in ice itself. When simpler hydrates $(12,13)$ such as $\mathrm{CaSO}_{4} \cdot 2 \mathrm{H}_{2} \mathrm{O}$ and $\mathrm{LiSO}_{4} \cdot \mathrm{H}_{2} \mathrm{O}$, were studied after irradiation by electrons at $77^{\circ} \mathrm{K}$ the number of inequivalent radical sites was much smaller, and the individual spectral lines were easy to resolve. Some of the results are shown in Figs. 3, 4, 5, and 6.

Irradiated calcium and lithium sulfates were first studied by Wigen and Cowen (14). The spectra in electron-irradiated samples of these salts, reported by these workers, showed two important types of paramagnetic centers. The first gave an isotropic line near the free electron $g$ value and was attributed to a trapped electron. The second gave anisotropic lines varying in hyperfine splitting from a few oersteds to a maximum of 21 Oe. These lines were attributed to a hole on the sulfate oxygen, which is hydrogen bonded to a water molecule.

The work discussed in the present paper forces a change in the interpretation of part of the irradiated sulfate spectra. While the existence of a center producing an isotropic line near the free electron $g$ value was verified, the experimental hyperfine splittings for the anisotropic line spectra found in this study differ by more than a factor of two from those reported by Wigen and Cowen. In order to understand the interpretation of this new data, it is first necessary to review the work done by Radford on OH radical produced in water. 
$\frac{d A}{d H_{0}}$ vs. MAGNETIC FIELD

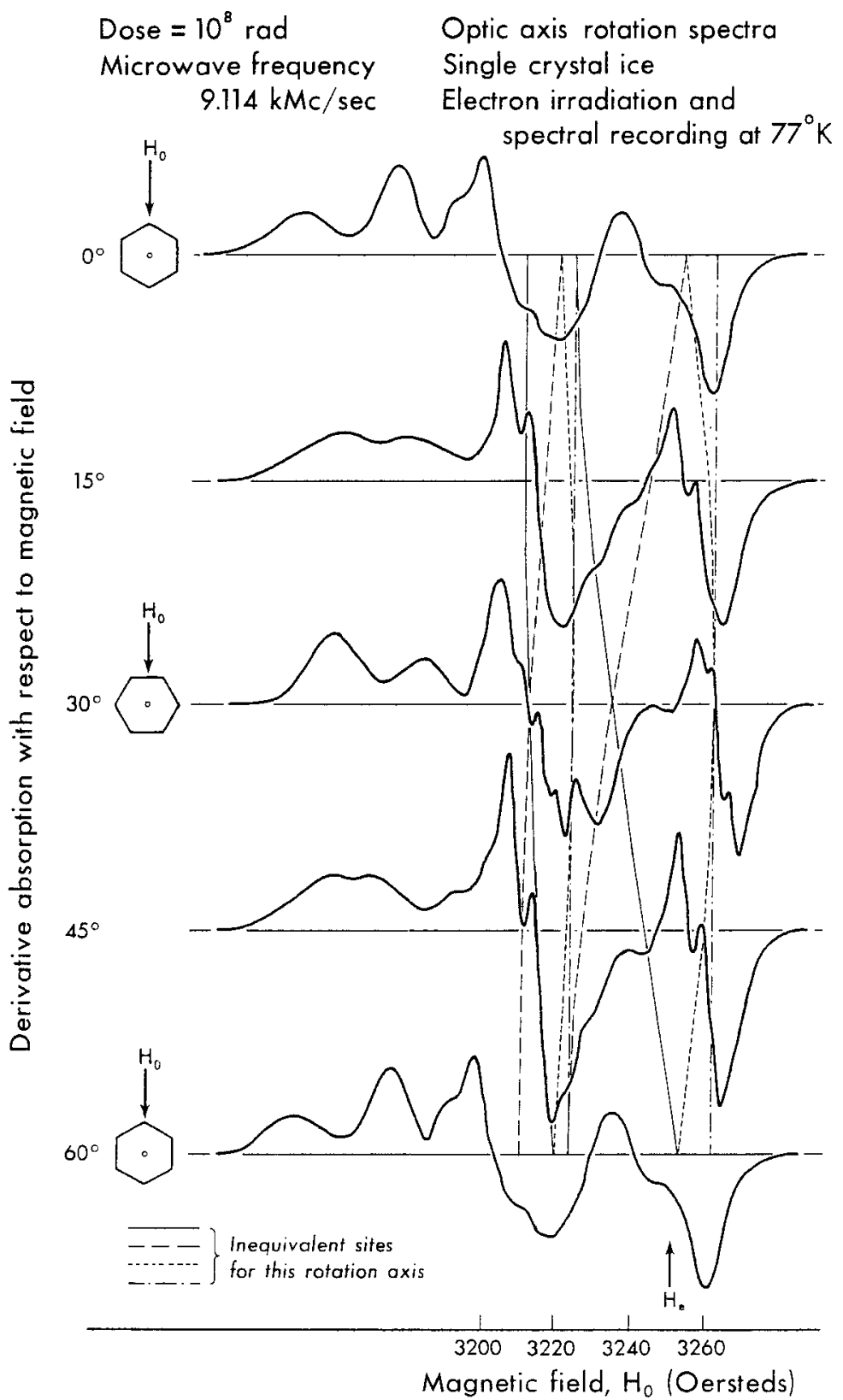

Figure 2. Single crystal ice derivative spectra for rotation about the crystal optic axis. 


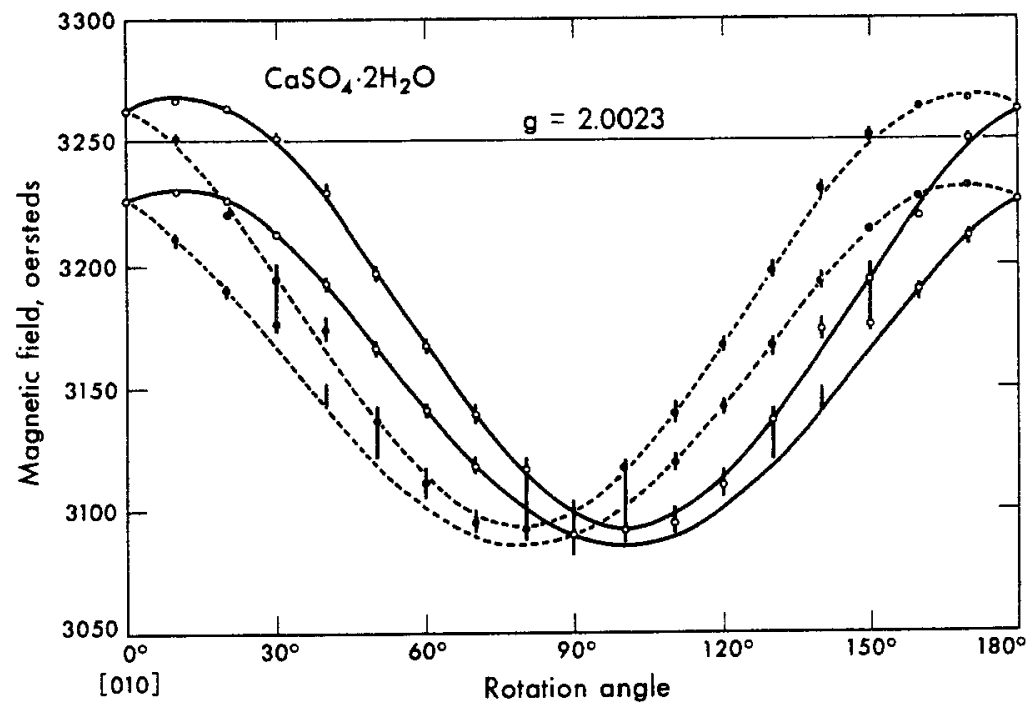

Figure 3. Magnetic field vs rotation angle for electronirradiated $\mathrm{CaSO}_{4} \cdot 2 \mathrm{H}_{2} \mathrm{O}$ at $77^{\circ} \mathrm{K}$. The spin Hamiltonian parameters are g 2.1108, g 2.0028, A $43 \mathrm{Oe}$,

A $32.5 \mathrm{Oe}, \mathrm{A} 3.3 \mathrm{Oe}$. MUB-3670

Figure 4. Magnetic field vs rotation angle for electronirradiated $\mathrm{Li}_{2} \mathrm{SO}_{4} \cdot \mathrm{H}_{2} \mathrm{O}$ at

$77^{\circ} \mathrm{K}$ for rotation about axis perpendicular to the crystal $b$ and $c$ axes. The spin Hamiltonian parameters are g 2.0065, g 2.0667, A $46 \mathrm{Oe}, \mathrm{A} 24 \mathrm{Oe}, \mathrm{A} 4 \mathrm{Oe}$. MUB-6477

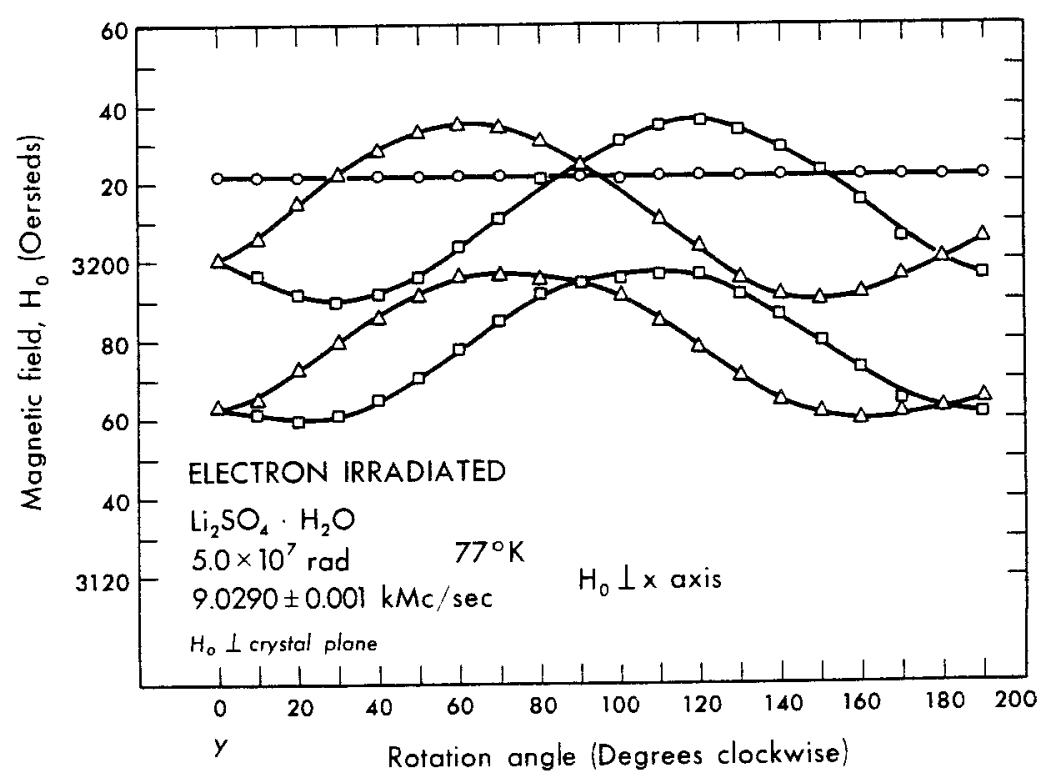




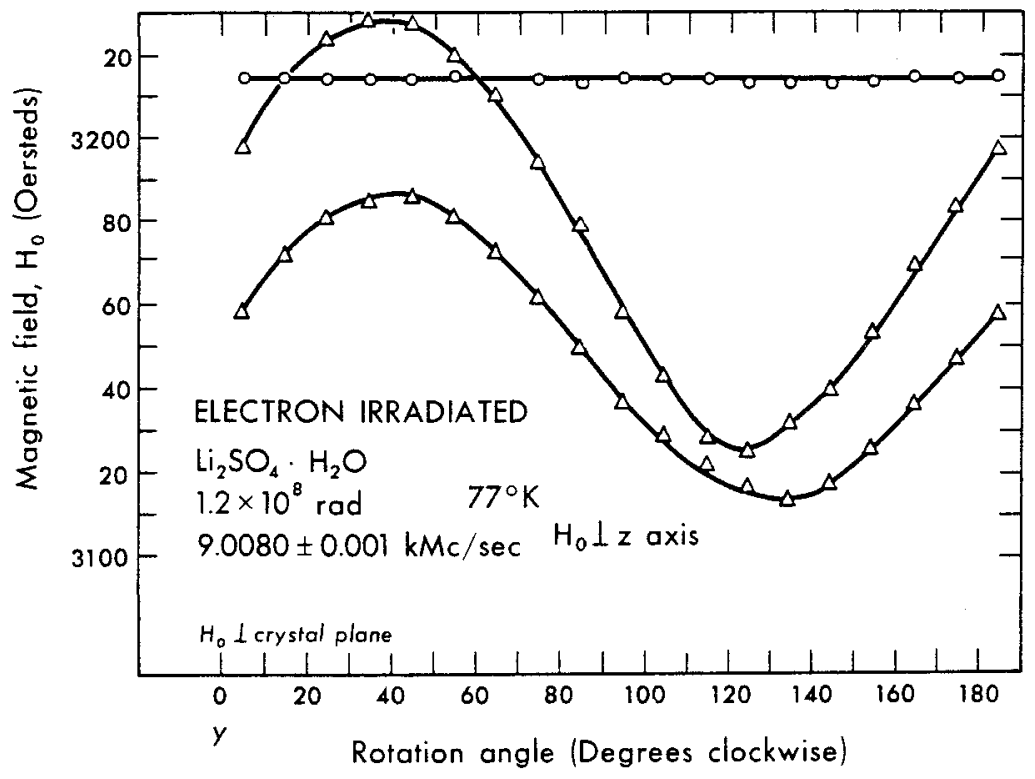

Figure 5. Magnetic field vs rotation angle for electronirradiated $\mathrm{Li}_{2} \mathrm{SO}_{4} \cdot \mathrm{H}_{2} \mathrm{O}$ at $77^{\circ} \mathrm{K}$ for rotation about crystal b axis.

Figure 6. Magnetic field vs rotation angle for electronirradiated $\mathrm{Li}_{2} \mathrm{SO}_{4} \cdot \mathrm{D}_{2} \mathrm{O}$ at $77^{\circ} \mathrm{K}$ for rotation about the crystal b axis. Spin Hamiltonian parameters are g $2.0065, \mathrm{~g} 2.0667$, A 7.1 Oe, A 3.7 Oe, A $0.6 \mathrm{Oe}$. MUB-6479

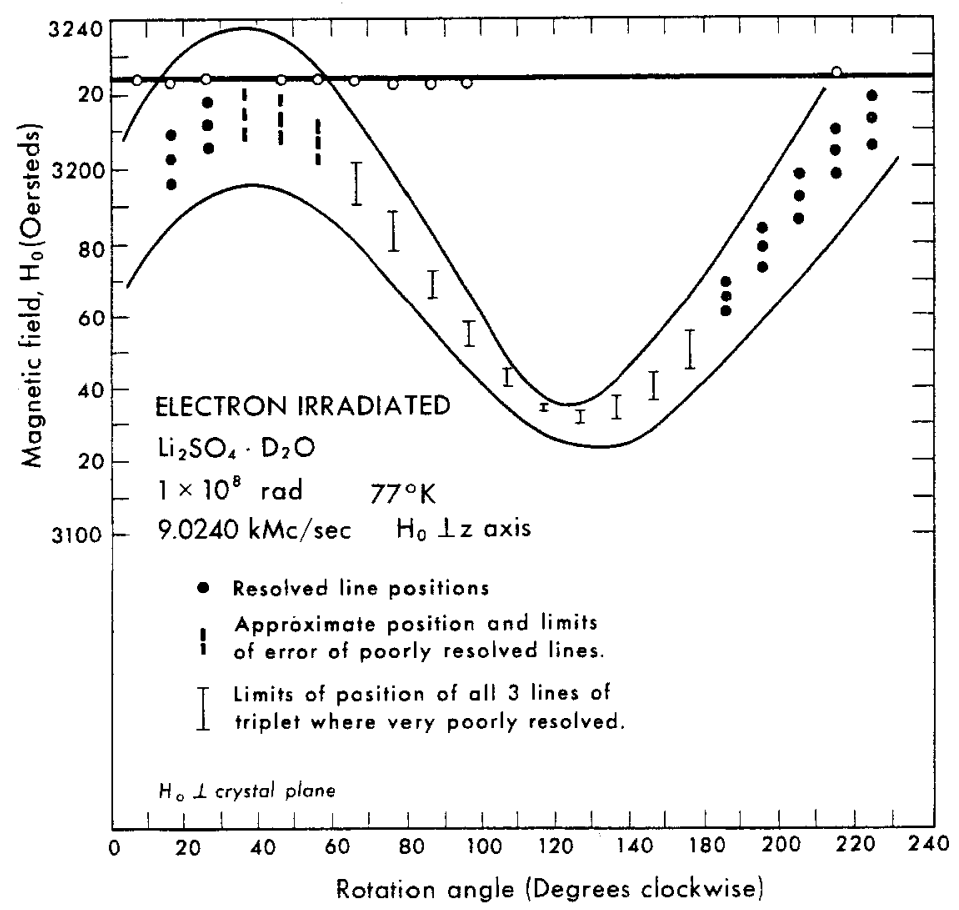


vapor by microwave discharge. While Radford's work is directed toward a physical understanding of the $\mathrm{OH}$ radical and is not a study of radicals produced by radiation, the results are invaluable in identifying $O H$ radical spectra in samples where it is produced by radiation.

OH free radical in the vapor phase was analyzed theoretically by Radford $(15,16)$ following the theory of Van Vleck (17) and of Dousmanis, Sanders and Townes (5) and extending this theory to cover the strong field Zeeman and hyperfine interactions. Optical- and microwave-absorption data (5) had shown $O H$ to be in a $\pi_{3 / 2}$ ground state. Wave functions for $\mathrm{OH}$ correct to electronic, vibrational, rotational and fine-structure interactions are determined by considering a case between Hunde's cases (A) and (B). g values were determined by considering the Zeeman interaction as a perturbation on a system with the above wave functions. The hyperfine interaction was treated as a perturbation on energy levels and wave functions correct to second order in Zeeman interaction.

The form used for the hyperfine interaction was that given by Frosch and Foley:

$$
H_{h f s}=a(I \cdot L)+(b+c) I_{z} S_{z}+\frac{1}{2} b\left(I^{+} S^{-}+I^{-} S^{+}\right)+\text {higher order terms }
$$

where $a, b$, and $c$ are coefficients that must be determined by experiment. In his second paper (16), Radford gives the values:

$$
\begin{aligned}
& b(O H)=-119.0 \pm 0.4 \mathrm{MC} / \mathrm{sec} \\
& c(O H)=133.2 \pm 1.0 \mathrm{MC} / \mathrm{sec}
\end{aligned}
$$

for the quantities of interest to this study as determined by his experiment.

The $g$ values observed by Radford in his experiment show very close agreement with those predicted by theory. The experimental values are thought to be good to three parts in $10^{5}$, and agreement of theory and experiment is of this same order. Hence there can be little doubt that Radford's theory and experiment describe the magnetic properties of the free OH molecule.

Radford's description of the hyperfine interaction in OH radical is emperical and is not compared to theory because of poor knowledge of the molecular orbitals of OH radical. Since the electron distribution functions for OH molecular orbitals are determined primarily by the Coulomb interaction between the electron in question and other electrons and nuclei, one would expect only small changes in the electron distribution functions because of perturbation interactions present in the case of OH trapped by hydrogen bonding in a solid lattice. Hence one would expect that the contact hyperfine interaction for $O H$, trapped either by hydrogen bonding to a sulfate group in irradiated sulfates and or by hydrogen bonding to an oxygen in irradiated ice, should differ from that observed by Radford by at most the ratio of hydrogen bond energies (for these hydrogen bonds) to Coulomb energies. This would be of the order of at most about ten percent. If one takes as the hyperfine interaction a combination of dipoledipole and contact hyperfine terms, this may be written for the case of axial symmetry as: 


$$
\underline{H}_{h f s}=-\frac{\left(\vec{\mu}_{S} \cdot \vec{r}\right)\left(\vec{\mu}_{I} \cdot \vec{r}\right)}{r^{5}}+\frac{\vec{\mu}_{S} \cdot \vec{\mu}_{I}}{r^{3}}-\frac{8 \pi}{3} \delta(\vec{r}) \vec{\mu}_{S} \cdot \vec{\mu}_{I}
$$

where $\vec{\mu}_{S}$ is the electron spin magnetic moment, $\vec{\mu}_{I}$ is the proton spin magnetic moment, $\vec{r}$ is the electron proton displacement and $\delta(r)$ is the Dirac delta function. Substituting

$$
\begin{aligned}
& \vec{\mu}_{S}=g \beta \vec{S} \\
& \vec{\mu}_{I}=\gamma \hbar \vec{I} \\
& a^{\prime}=\frac{8 \pi}{3} \delta(\vec{r})
\end{aligned}
$$

one obtains the form

$$
\underline{H}_{h f s}=g \beta \gamma \hbar\left\{\frac{3(\vec{S} \cdot \vec{r})(\vec{I} \cdot \vec{r})}{r^{5}}-\frac{\vec{s} \cdot \vec{I}}{r^{3}}+a^{\prime} \vec{s} \cdot \vec{I}\right\}
$$

where $g$ is the spectroscopic splitting factor, $\beta$ is the Bohr magnetron, $\vec{S}$ is the electron spin angular momentum operator, $\gamma$ is the proton gyromagnetic ratio and $\vec{I}$ is the nuclear spin angular momentum operator.

In tensor form this interaction may be written as

$$
\underline{H}_{h f s}=g \beta \gamma \hbar(\vec{S})\left(\begin{array}{ccc}
\frac{3 x^{2}-r^{2}}{r^{5}}+a^{\prime} & \frac{x y}{r^{5}} & \frac{x z}{r^{5}} \\
\frac{x y}{r^{5}} & \frac{3 y^{2}-r^{2}}{r^{5}}+a^{\prime} & \frac{y z}{r^{5}} \\
\frac{x z}{r^{5}} & \frac{y z}{r^{5}} & \frac{3 z^{2}-r^{2}}{r^{5}}+a^{\prime}
\end{array}\right)(\vec{I})
$$

where $(\vec{S})$ is now looked upon as a row vector and $(\vec{I})$ as a column vector. Since the tensor above is real, its trace is invariant to rotation of the coordinate system. Hence one may write the value of the trace of the above interaction as

$$
\text { Trace } \underline{H}_{h f s}=3 g \beta \gamma \hbar a^{\prime}(\overline{(\vec{S} \cdot \vec{I})} \text {. }
$$

Similar arguments may be used in the case of $A_{x x} \neq A_{y y}$ (nonaxial symmetry) to show that if the hyperfine interaction is written in the general form

$$
\underline{H}_{h f s}=(\vec{S})\left(\begin{array}{ccc}
A_{x x} & 0 & 0 \\
0 & A_{y y} & 0 \\
0 & 0 & A_{z z}
\end{array}\right)
$$

in the principle axis system, the contact interaction is one-third the sum of the diagonal elements. This is used to determine the contact interaction for the anisotropic lines in the irradiated hydrated crystals. 
The experimental results, found in the case of calcium and lithium sulfates with the corresponding effective spin Hamiltonian parameters calculated from the data, are given in Figs. 3-5. A comparison of the measured, value of the contact hyperfine interaction found in these centers with that found for $\mathrm{OH}$ in vapor by Radford is shown in Table 1 below. Figure 6 shows the result of deuteration of lithium sulfate on the EPR spectra observed after irradiation by $6 \mathrm{MeV}$ electrons. It should be noted that the ratio of the splitting of the lines of the doublet observed in the hydrate case to the splitting of two nearest field position lines in the deuterate case, at an orientation having good line resolution, is very nearly 6.5 , the ratio of proton to deuteron moments.

Table 1

\begin{tabular}{lc}
\hline Sample & $\begin{array}{c}\text { Isot ropic hyperfine } \\
\text { splitting }\end{array}$ \\
\hline 1) $\mathrm{OH}$ in vapor (Radford) & $26.7 \mathrm{Oe}$ \\
2) $\mathrm{OH}$ in $\mathrm{CaSO}_{4} \cdot 2 \mathrm{H}_{2} \mathrm{O}$ & $26.3 \mathrm{Oe}$ \\
$\quad(\mathrm{Gunter}$ and Jeffries) & \\
3) $\mathrm{OH}$ in $\mathrm{Li}_{2} \mathrm{SO}_{4} \cdot \mathrm{H}_{2} \mathrm{O}$ & 24.7 Oe \\
(this paper) & 3.79 Oe or $24.7 / 6.5$ \\
4) $\mathrm{OD}$ in $\mathrm{Li}_{2} \mathrm{SO}_{4} \cdot \mathrm{D}_{2} \mathrm{O}$ & \\
(this paper) &
\end{tabular}

A study of the orientation of the symmetry axes of the $g$ tensors of the anisotropic centers found in the irradiated sulfates shows that these axes lie within about $5^{\circ}$ of the nearest cation to sulfate oxygen direction in these crystals. This might be expected for OH radical held on the sulfate oxygen by a hydrogen bond if the other end oriented itself so that its oxygen was directed toward the cation.

It appears almost certain then that the anisotropic centers observed in the irradiated sulfates are $O H$ radical centers trapped by hydrogen bonding to the sulfate oxygen. The hyperfine doublet for the hydrate and triplet for the deuterate with a splitting smaller by a factor of 6.5, the good agreement of the observed contact hyperfine interaction with that observed for $\mathrm{OH}$ in the vapor phase and the reasonable position of the symmetry axes for the observed centers all support this identification.

In addition to the $\mathrm{OH}$ centers discussed above and the trapped electron centers first identified by Wigen and Cowen, weak hydrogen atom lines were also seen in the EPR spectra of the irradiated sulfates. The observed splittings were 513 Oe in the calcium sulfate case and 506.5 Oe in the lithium sulfate case.

It should be noted then that all three paramagnetic species suspected of being involved in the early disintegration reactions of $\mathrm{H}_{2} \mathrm{O}$ are present in the irradiated sulfate crys tals. This should then be an extremely interesting system in which to study radical yields at low temperature. From a study of this system at liquid helium temperature, for example, it may be possible to determine the relative number of water molecules breaking up through the 
Samuel-McGee process and those breaking up through the Lea-Gray-Platzmann Process. This work is now in progress.

As is shown in Fig. 2, effective spin Hamiltonian parameters for OH in irradiated ice have been worked out following the lead given by the study of OH in irradiated crystal hydrates. In both cases the $\mathrm{OH}$ is believed to be held in the crystal by hydrogen bonding to oxygen. Because in the ice case there are four hydrogen bonds as opposed to a single hydrogen bond in the case of the irradiated crystal hydrates studied, quenching of orbital angular momentum should be more complete in the ice case. This is verified by the data and their description through the given effective spin Hamiltonian parameters.

The experimentally determined effective spin Hamiltonian parameters

$$
\begin{array}{ll}
\mathrm{g}_{\|}=2.026 & \mathrm{~A}_{\mathrm{zz}}=4 \mathrm{Oe} \\
\mathrm{g}_{\perp}=2.008 & \mathrm{~A}_{\mathrm{xx}}=\mathrm{A}_{y y}=38 \mathrm{Oe}
\end{array}
$$

predict a low field component of the hyperfine doublet almost stationary with respect to magnetic field value upon crystal rotation. The predicted position of the high field line in the perpendicular orientation is near the observed position of the high field line in polycrystalline ice spectra. Hence these effective spin Hamiltonian parameters predict a polycrystalline ice spectrum like that observed for polycrystalline ice.

These effective spin Hamiltonian parameters, which differ from those given by McMillan et al. (6), are thought to be correct because:

1) The interpretation assumes $O H$ to be bonded along the original hydrogen bond directions.

2) This effective spin Hamiltonian completely accounts for that part of the irradiated polycrystalline ice spectrum associated with $O H$ radical.

3) A contact hyperfine interaction of 26.7 Oe in close agreement with that expected for $\mathrm{OH}$ is found.

4) This effective spin Hamiltonian fits the single crystal spectral data as well as could be expected for spectra of such complexity.

Radical yield studies for $O H$ radical in polycrystalline ice have been carried out over a wide range of dose at $77^{\circ} \mathrm{K}$ by techniques discussed above. Over the dose ranges where Siegel makes similar measurements, the two results are in good agreement. These results are shown in Fig. 7 .

The observed saturation of the radical yield at high doses may be explained by a local heating model. At the time of its creation the $O H$ radical is in a zone in the crystal lattice which is "hot" because of the dissipating radiation energy. It may then migrate through the crystal by hydrogen transfer or by localized melting of the crystal and radical diffusion until the zone cools and this transfer or diffusion is no longer favored. After a certain radical concentration is built up there is a high probability that this radical migration may lead to 


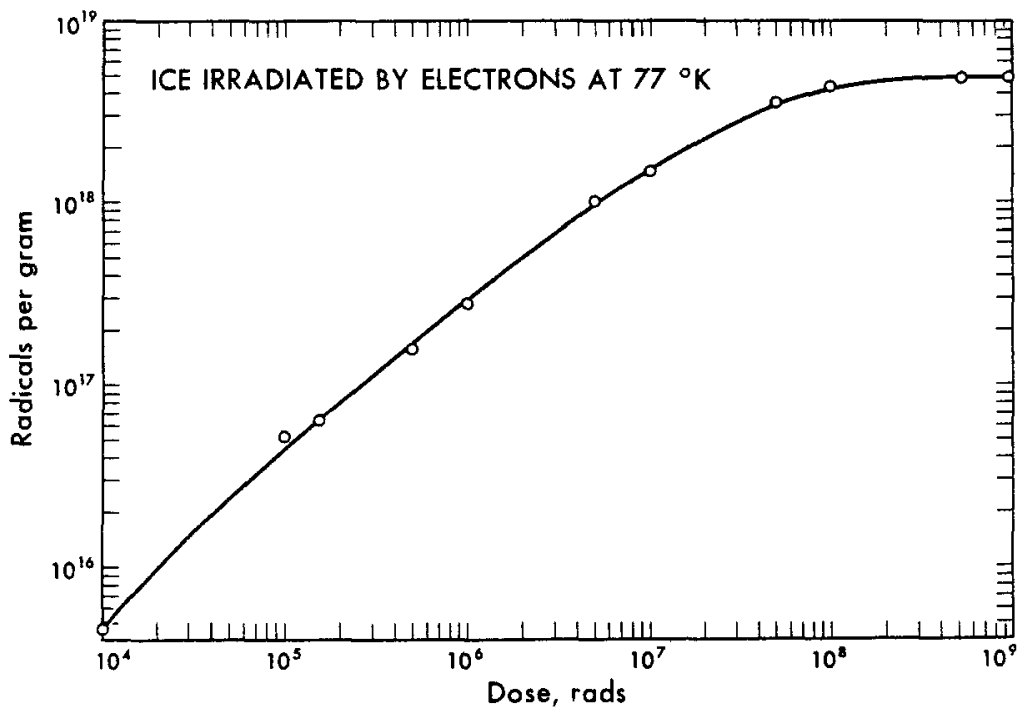

Figure 7. Radical yield vs dose for $\mathrm{OH}$ radical in electron-irradiated polycrystalline ice at $77^{\circ} \mathrm{K}$. $\mathrm{MUB}-8562$

recombination of radicals at the same rate that they are being produced. At this concentration the radical yield should show complete saturation.

\section{SUMMARY}

Studies on the EPR spectra produced by irradiation of $\mathrm{H}_{2} \mathrm{O}$ in single crystals of ice and in several crystal hydrates have been carried out. Effective spin Hamiltonian parameters have been determined for a radical identified as OH. These results differ from previously published results on the samples studied. OH radical yields over a wide range of dose have been made.

\section{ACKNOWLEDGMENTS}

The author wishes to express his gratitude to Prof. C. D. Jeffries, Prof. C. A. Tobias, Prof. S. J. Wyard, Dr. T. Henriksen and Prof. W. T. Doyle, for advice and for many helpful discussions useful in this work. Special thanks are given to D. W. Pounds for help in many of the experiments and discussions of the experimental apparatus while using the facilities of the LRL Electron Linear Accelerator.

\section{REFERENCES}

1. Siegel, S.; Baum, L. H.; Skolnik, S., and Flournoy, J. M.; J. Chem. Phys. 32: 1249, 1960.

2. Siegel, S.; Flournoy, J. M., and Baum, L. H.; J. Chem. Phys. 34: 1782, 1961.

3. Judeikis, H. S.; Flournoy, J. M. , and Siegel, S.; J. Chem. Phys. 37: 2272, 1962.

4. Siegel, S.; J. Chem. Phys. 39: 390, 1963.

5. Dousmanis, G. C.; Sanders, T. M., and Townes, C. H.; Phys. Rev. 100: $1735,1955$.

6. McMillan, J.A.; Matheson, M. S., and Smaller, B.; J. Chem. Phys. 33: 609, 1960 .

7. Barnes, W. H.; Proc. Roy. Soc. A 125: 670, 1929. 
8. Peterson, S. W., and Levy, H. A. ; Acta Cryst, 10: 70, 1957.

9. Jona, F., and Scherrer, P.; Helv. Phys. Acta 25: 35, 1952.

10. Gere, E. A.; unpublished report.

11. Gunter, T. E., and Jeffries, C. D.; UCRL-11387: 8, 1964.

12. Wooster, W. A.; Z. Krist. 94: 375, 1936.

13. Ziegler, G. E.; Z. Krist. 89:456, 1934.

14. Wigen, P. E., and Cowen, J. A.; J. Phys. Chem. Solids 17: 26, 1960.

15. Radford, H. E.; Phys. Rev. 122: 114, 1961.

16. Radford, H. E.; Phys. Rev. 126: 1035, 1962.

17. Van Vleck, J. H. ; Phys. Rev. 33: 467, 1929.

Received November, 1965. 


\title{
Reticuloendothelial Activity in Neonatally Thymectomized Mice and Irradiated Mice Thymectomized in Adult Life
}

\author{
John C. Schooley, Lola S. Kelly, Ernest L. Dobson, Caroline R. Finney, \\ Virginia W. Havens and Linda N. Cantor
}

In the last few years, considerable evidence has accumulated supporting evidence for the importance of the thymus in the initial development of immunological competence and in the reestablishment of immunological reactivity in the adult animal after the immune response has been suppressed by whole-body irradiation, Miller (1). Phagocytosis of antigen as well as the production of specific antibody occur in the normal mouse following the injection of particulate antigen. In Iymphoid tissue, the juxtaposition of phagocytic cells containing antigen and cells synthesizing antibody against the antigen has suggested an interrelationship between phagocytosis of the antigen and the production of antibody. Indeed, many theories of antibody formation either propose or imply phagocytosis of the antigen as a first step in the inductive phase of the immune response. This paper deals with the phagocytic capacity of the reticuloendothelial system of mice whose immunologic reactivity was suppressed either by neonatal thymectomy or by a combination of adult thymectomy, whole-body irradiation and bone-marrow transplantation. An abstract of some of this work has been published, Schooley et al. (2).

\section{METHODS}

Male $\mathrm{LAF}_{1}$ mice were thymectomized or sham-operated within $8 \mathrm{hr}$ after birth; female $\mathrm{C}_{3} \mathrm{H}$ mice were thymectomized or sham-operated at seven to nine weeks of age. Three weeks later the $\mathrm{C}_{3} \mathrm{H}$ mice were irradiated with $960 \mathrm{R}$ whole-body ${ }^{60} \mathrm{Co}$ gamma rays. Immediately after irradiation, $9 \times 10^{6}$ viable (eosin excluding) bone-marrow cells obtained from the femurs and tibias of isologous donor mice were injected intravenously in $0.5 \mathrm{ml}$ of Tyrode's solution. Precautions were taken to insure that the animals were kept in a clean environment, i.e., small numbers of mice were housed in frequently changed, sterilized cages, and the drinking water was chlorinated (14 ppm).

The phagocytic function of the reticuloendothelial system was determined at $70-120$ days of age in the group of mice thymectomized at birth, and at 30 days post-irradiation in the group of mice thymectomized as adults. The rate of disappearance from the blood of intravenously injected colloidal carbon was measured following the method employed extensively by Halpern, Benacerraf, Biozzi and their coworkers, see Benacerraf et al. (3). The mice were restrained, and a tail vein was injected with $0.1 \mathrm{ml}(6 \mathrm{mg})$ of a suspension of colloidal carbon (Gunther Wagner, Hanover, No. C 11/1431a, prepared, as described by Parker and Finney (4). Blood samples of $5 \mu 1$ were taken from the tip of the tail approximately every $5 \mathrm{~min}$ for $30 \mathrm{~min}$ and diluted in $1 \mathrm{ml}$ of $0.02 \mathrm{~N} \mathrm{NaOH}$. The carbon concentration was meas ured as optical density, read at $750 \mathrm{m \mu}$ on a Beckman Model DU spectrophotometer. For 
each mouse, the disappearance rate constant $\left(k=0.693 / t_{1 / 2}\right)$ was determined from a semilogarithmic plot of the carbon concentration as a function of time. The constant $k$ represents the actual fraction of the carbon present in the blood, which disappears from the circulation per minute. In Tables 1 and 2, values of $\mathrm{k}$ have been expressed as percent per minute; i.e., values given are $\mathrm{k} \times 100$, where $\mathrm{k}$ is defined by the exponential function $\mathrm{C}=\mathrm{C}_{\mathrm{o}} e^{-\mathrm{kt}}$.

Table 1. Reticuloendothelial system activity in mice thymectomized at birth

\begin{tabular}{lcccc}
\hline & $\begin{array}{c}\text { No. of } \\
\text { mice }\end{array}$ & $\begin{array}{c}\mathrm{k} \\
\text { \% per min }\end{array}$ & $\begin{array}{c}\text { Spleen } \\
\text { \% body weight }\end{array}$ & $\begin{array}{c}\text { Liver } \\
\text { \% body weight }\end{array}$ \\
\hline Control & 33 & $2.39 \pm 0.03$ & $0.35 \pm 0.004$ & $4.7 \pm 0.16$ \\
All thymectomized & 38 & $4.32 \pm 0.90$ & $0.82 \pm 0.12$ & $5.6 \pm 0.19$ \\
$\begin{array}{l}\text { Thymectomized } \\
\text { non-runts }\end{array}$ & 23 & $3.24 \pm 0.59$ & $0.42 \pm 0.03$ & $5.1 \pm 0.10$ \\
All runts & 15 & $6.69 \pm 2.4$ & $1.06 \pm 0.27$ & $6.3 \pm 0.25$ \\
Runty runts & 6 & $12.3 \pm 4.7$ & $1.52 \pm 0.45$ & $6.5 \pm 0.30$ \\
\hline
\end{tabular}

Indicated errors are standard errors of the means.

Table 2. Reticuloendothelial system activity in mice thymectomized as adults

\begin{tabular}{|c|c|c|c|c|}
\hline & $\begin{array}{c}\text { No. of } \\
\text { mice }\end{array}$ & $\stackrel{\mathrm{k}}{\% \text { per min }}$ & $\begin{array}{l}\text { Spleen } \\
\% \text { body weight }\end{array}$ & $\begin{array}{c}\text { Liver } \\
\% \text { body weight }\end{array}$ \\
\hline $\begin{array}{l}\text { Control } \\
\text { Unoperated }\end{array}$ & 15 & $2.42 \pm 0.12$ & $0.474 \pm 0.021$ & $5.28 \pm 0.10$ \\
\hline $\begin{array}{l}\text { Sham thymectomized, } \\
\text { Irradiated, bone } \\
\text { marrow transplanted }\end{array}$ & 16 & $2.47 \pm 0.14$ & $0.525 \pm 0.078$ & $5.78 \pm 0.12$ \\
\hline $\begin{array}{l}\text { Thymectomized, } \\
\text { Irradiated bone } \\
\text { marrow transplanted }\end{array}$ & 11 & $3.10=0.22$ & $0.433 \pm 0.025$ & $5.87 \pm 0.15$ \\
\hline
\end{tabular}

Indicated errors are standard exrors of the means.

Following the measurement of carbon clearance, the spleen and liver were removed, weighed, fixed in Bouin's fluid and prepared for histological examination. The completeness of thymectomy was determined, and mice having remnants of thymic tissue were excluded from the experiments. Survival time of homografts was tested in a small series of mice by grafting $\mathrm{C}_{3} \mathrm{H}\left(\mathrm{H}-2^{\mathrm{k}}\right)$ ear skin on the back of neonatally thymectomized $\mathrm{LAF}{ }_{1}\left(\mathrm{H}-2^{\mathrm{b}} / \mathrm{H}-2^{\mathrm{a}}\right)$ mice. The grafts were recorded as rejected when the last living part of the epithelium became necrotic. Fifty percent hemolysin titers in the serum of another small series of neonatally thymectomized mice were determined nine days after the intraperitoneal injection of $0.2 \mathrm{ml}$ of a $10 \%$ suspension of sheep red blood cells. 


\section{RESULTS}

Great variability was observed in the rate of carbon clearance when neonatally thymectomized mice were considered as a single group. Many of the thymectomized mice were indistinguishable from the controls in appearance and differed relatively little from them in the rate of carbon clearance. However, it was apparent that a small number of the mice, which were severely runted, had very active reticuloendothelial systems and cleared carbon at rapid rates. This suggested that the thymectomized group should be further subdivided into groups based on the degree of runting. Since the degree of runting was difficult to assess quantitatively, age versus weight was plotted for control animals and a growth curve was drawn on this plot in such a way that essentially all normal animals were above the line. A similar plot of age versus weight was made for neonatally thymectomized mice. On the basis of the growth curve of normal animals, neonatally thymectomized mice were divided into two groups. One group of 23 mice was indistinguishable from the control group on an age-weight basis. These mice were designated thymectomized non-runts. Fifteen mice weighed less for their age than control mice and were designated runts. These relationships are shown in Fig. 1. Six of the 15 runted mice, termed "runty runts" (encircled in Fig. 1), had weights far below control mice and demonstrated, in addition, signs described as characteristic of the runting syndrome, Parrott and East (5), and Miller (6), i. e., lethargy, hunched posture, ruffled hair and a generally emaciated condition.

The values of $k$ for carbon clearance and the spleen and liver weights of the shamoperated controls and of the neonatally thymectomized miceare given in Table 1. A significant difference in the values for the colloidal carbon disappearance rate constant, $k$, was observed between the thymectomized group and the controls $(P<0.025)$. This difference becomes much more pronounced when the runted mice are considered separately. Indeed, the $k$ for the "runty runt" group is five times the $k$ for the controls $(P<0.001)$. The thymectomized non-runts cleared carbon somewhat faster than the controls, but the difference is not highly significant $(P>0.05)$.

The spleen and liver weights given in Table 1 are expressed as percent body weight. The spleen and liver weights of the thymectomized animals considered as a single group are significantly higher than the control mice, but again this is largely a reflection of the very large relative weight increase of these organs in the thymectomized mice classified as runts. The spleens of the "runty runt" group were about five times larger than control spleens on the basis of percent body weight, and even in terms of absolute size these spleens were about 2.5 times larger than the controls.

The values of $k$ for carbon clearance and the spleen and liver weights of mice thymectomized as adults, lethally irradiated, and transplanted with isologous bone marrow and their sham-operated controls are given in Table 2. The values of normal mice of the same age are also given. The thymectomized group cleared carbon slightly faster than the shamoperated controls. However, a quarter of the thymectomized animals died very suddenly 25 to 30 days after irradiation, and consequently carbon clearance rates could not be measured in these animals. The spleen and liver weights of the thymectomized and sham-operated 


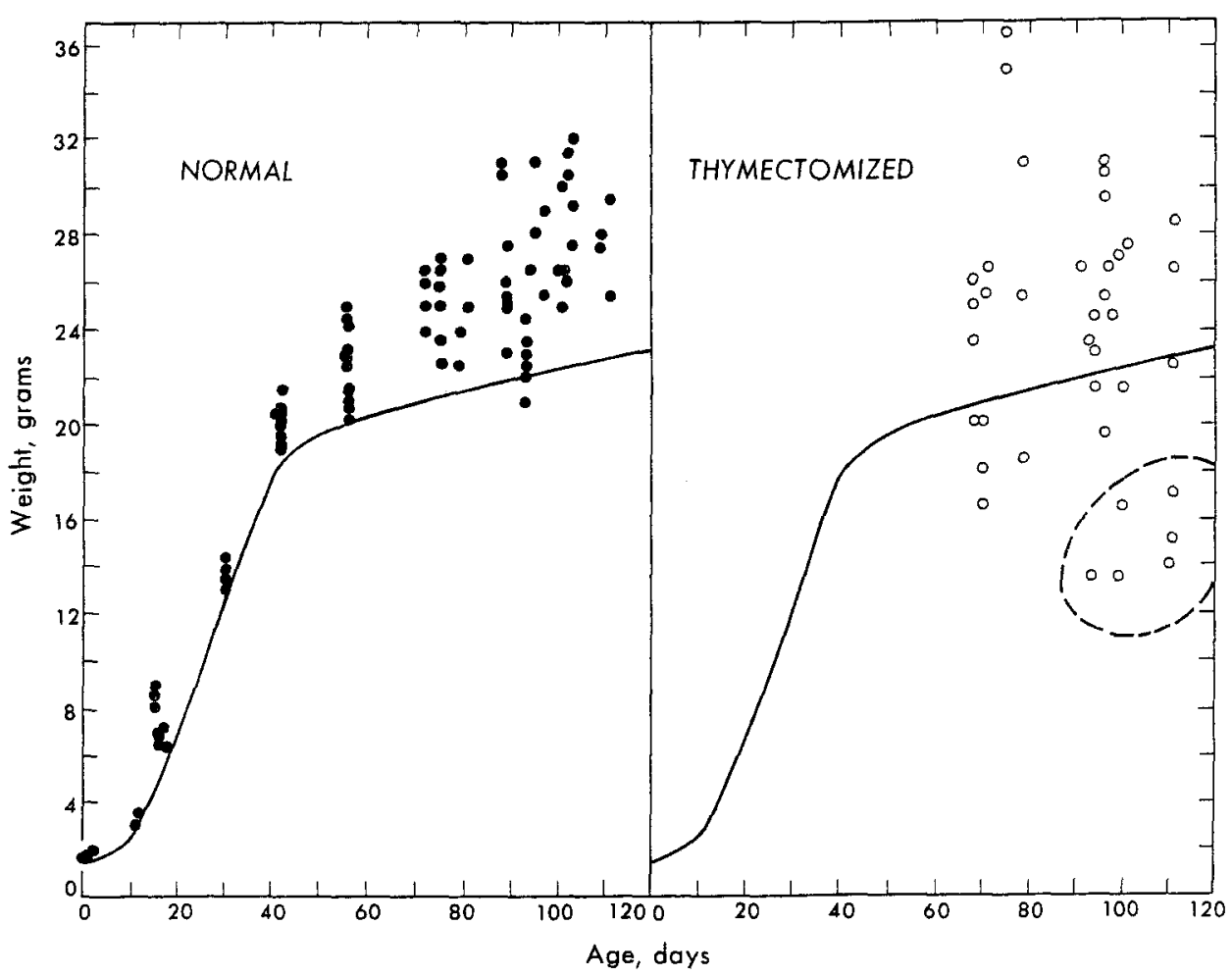

Figure 1. Weight has been plotted as a function of age for both control and neonatally thymectomized mice. A curve has been drawn so that essentially all of the control animals fall above the line. The thymectomized mice above the line are the non-runts; all animals below the line are considered runts; the animals enclosed by the dotted line were included in the runt group but have also been considered separately as "runty runts". MUB-3201

groups are similar, but the liver weights of these irradiated groups are greater than the unirradiated controls $(\mathrm{P}>0.0025)$.

The histologic appearance of the spleens of the neonatally thymectomized mice was variable. In general, the white pulp of the spleen was well demarcated in thymectomized as well as in normal animals by the phagocytized carbon in the perifollicular zone. It was our impression that the follicles of the spleens of thymectomized animals were depleted of lymphoid elements, particularly small lymphocytes. In some cases this depletion was pronounced. In some (but not all) of the spleens of the runts, the architecture of the enlarged spleens was disorganized, and only an occasional area could be classified as follicular. These follicles were small and relatively acellular. These enlarged and disorganized spleens appeared to contain less carbon than the control mice. This decreased carbon content seemed to be greater than could be accounted for by the dilution effect of splenic enlargement. Occasionally in the thymectomized animals, large accumulations of plasma cells were observed. These observations are generally consistent with the observations of other authors, Miller (6), de Vries et al. (7) and Miller and Howard (8). 
The histological appearance of the spleens in irradiated thymectomized adult mice one month after bone-marrow transplantation was also somewhat variable. In almost all of the spleens, follicles were present and well demarcated by phagocytized carbon. Fewer and smaller follicles were present in the spleens of the thymectomized irradiated mice than in the spleens of the sham-operated control mice. These sham-operated irradiated mice, however, appeared to have fewer and smaller follicles than the unirradiated control mice. Few small lymphocytes were present in the follicles of the spleen in the thymectomized mice. In some of the spleens of the thymectomized mice, an extreme atrophy of the white pulp was observed and the follicular structure was virtually absent. A decreased content of phagocytized carbon was evident in these spleens, but no evident correlation with the rate of carbon clearance was found. Except for the absence of marked splenomegaly, essentially the same histopathologic changes were observed in the spleens of this group as in the spleens of the neonatally thymectomized group.

The 50\% hemolysin titers of the serum in neonatally thymectomized mice, although not abolished, were significantly below the values found in unoperated or sham-operated control mice as indicated in Table 3. The hemolysin titers of the sham-thymectomized mice were slightly lower than unoperated controls, which suggests that the stress of the sham operation in some way influenced the subsequent development of immunological reactivity. The hemolysin titers of irradiated adult thymectomized mice transplanted with bone marrow were determined in another series of mice, and it was found that the titers were depressed and similar to those found in neonatally thymectomized mice.

Table 3. 50\% hemolysin titers of mice thymectomized at birth

\begin{tabular}{lcc}
\hline Treatment & No. of mice & $50 \%$ hemolysin titer \\
\hline Unoperated & 11 & $314 \pm 45$ \\
$\begin{array}{l}\text { Sham-thymectomized } \\
\text { at birth }\end{array}$ & 7 & $232 \pm 23$ \\
$\begin{array}{l}\text { Thymectomized } \\
\text { at birth }\end{array}$ & 21 & $138 \pm 21$ \\
\hline
\end{tabular}

Indicated errors are standard errors of the means.

The survival of homografts in the neonatally thymectomized mice was much prolonged over both sham -thymectomized and unoperated control mice as shown in Table 4. Miller et al, (9) and Cross et al. (10) have demonstrated that homograft rejection is also markedly prolonged in irradiated mice thymectomized in adult life.

\section{DISCUSSION}

A decided increase was observed in the phagocytic activity of neonatally thymectomized mice compared to control animals. However, when the thymectomized mice were further subdivided into groups according to the degree of runting, the most marked increases in 
Table 4. Survival of homografts $\left(\mathrm{C}_{3} \mathrm{H}\right)$ in $\mathrm{LAF}_{1}$ mice thymectomized at birth

\begin{tabular}{|c|c|c|c|c|c|}
\hline \multirow{2}{*}{ Treatment } & \multirow{2}{*}{$\begin{array}{l}\text { Number } \\
\text { of mice }\end{array}$} & \multicolumn{4}{|c|}{ Survival time of skin graft } \\
\hline & & $<14$ days & $14-30$ days & $31-60$ days & $>60$ days \\
\hline Unoperated & 14 & 2 & 12 & 0 & 0 \\
\hline $\begin{array}{l}\text { Sham-thymectomized } \\
\text { at birth }\end{array}$ & 4 & 1 & 3 & 0 & 0 \\
\hline $\begin{array}{l}\text { Thymectomized } \\
\text { at birth }\end{array}$ & 7 & 0 & 2 & 3 & 2 \\
\hline
\end{tabular}

phagocytic activity were found in the more severely runted mice. The neonatally thymectomized non-runted mice, which were indistinguishable from control mice on an age-weight basis, had phagocytic activities only slightly greater than control mice.

Only a moderate increase in phagocytic activity was observed in irradiated thymectomized adult mice. In contrast to the neonatally thymectomized mice, animals exhibiting the characteristic runting syndrome, including splenomegaly and hepatomegaly, did not occur in the group thymectomized as adults.

We suggest that, since the correlation between the degree of runting and reticuloendothelial activation is much better than the correlation between thymectomy and reticuloendothelial activation, the stimulation to greater phagocytic activity is only indirectly related to the impairment of the immune response resulting from thymectomy and is more dir ectly related to the as yet unknown factors responsible for runting.

The pathogenesis of the runting syndrome following perinatal thymectomy is a perplexing problem. There is considerable variation in the incidence of runting in different strains of mice and in the same strain in different laboratories. We observe that only about ten percent of our neonatally thymectomized LAF 1 mice exhibit the characteristic runting syndrome. Parrott and East (11) were initially unable to isolate any specific bacterial or viral agents from the organs or blood of neonatally thymectomized runted mice. More recently, they (East et al. (12)) have observed lesions in the liver of a small number of thymectomized mice caused by a hepatotrophic virus. They consider, however, that a role of the virus in the production of runting is problematic. Such liver lesions were not observed in the thymectomized mice used in the present experiment, although small areas of granulocytic infiltration were observed occasionally.

Howard (13) has demonstrated a marked increase in reticuloendothelial system activity associated with the graft-versus-host reaction. The similarity between the runting syndrome observed following neonatal thymectomy and the wasting syndrome of the graftversus-host reaction suggests a common pathogenesis. Indeed, de Vries and coworkers (7) have postulated from a histo-pathological study that runting in thymectomized mice may be 
the result of an autoimmune response with infection as one of the major causes of death. Wilson et al. (14) and McIntire et al. (15) have found that the runting syndrome did not occur in neonatally thymectomized germfree strains of mice, although a high incidence of runting occurs in the same strains in a conventional environment. In addition, McIntire and coworkers found that the wasting syndrome of graft-versus-host reactions did occur in germfree mice. In contrast, Wilson and Connell (16) did not observe pathologic changes characteristic of the secondary disease of the graft-versus-host reaction in germfree mice. Thus, the evidence on the equivalence of these syndromes is conflicting at the present time. In regard to the runting syndrome seen in neonatally thymectomized mice, the available evidence certainly suggests that either infectious agents or other, noninfectious, antigens which have been minimized or eliminated by the germfree environment, induce the syndrome. Regardless of the precise manner in which the runting syndrome is initiated, our results indicate an excellent correlation between the degree of runting and reticuloendothelial system activation. Furthermore, in the absence of runting, there is very little correlation between immunological incompetence produced as a result of thymectomy and reticuloendothelial system activation. Certainly there was no evidence of a hypoactive reticuloendothelial system in any of the groups of thymectomized animals. It should be emphasized, however, that the measurement of the clearance of carbon from the blood is primarily a measurement of the phagocytic activity of that portion of the reticuloendothelial system which is present in the liver.

While this manuscript was in preparation, the results of a similar study were pre sented by Miller and Howard (8). Our results essentially confirm those of Miller and Howard on neonatally thymectomized animals and extend the observations to include adult thymectomized and irradiated animals.

In rats, Morrow and Di Luzio (17) found that the phagocytic activity was not significantly altered by neonatal thymectomy. Their rats showed no evidence of runting or splenomegaly. We too have made measurements (previously unpublished) on neonatally thymectomized male Buffalo/N rats that were considerably smaller and presumably younger than the rats used by Morrow and Di Luzio. These rats averaged $100 \mathrm{~g}$ body weight, and the thymectomized group was indistinguishable from the control group on an age-weight basis. No runts were observed in this group, although an occasional runt had occurred in an earlier large series. The disappearance constant for 16 control rats was $0.199 \pm 0.051$ and for 13 thymectomized rats was $0.297 \pm 0.049$. This slight increase in carbon clearance rate for the thymectomized rats is not significant $(P>>0.1)$. Thus, in rats that runt only very rarely, our data and those of Morrow and Di Luzio do not indicate a significantly increased phagocytic activity. This supports our suggestion that the increased reticuloendothelial system activity, observed in thymectomized mice, is related to the presence of some factor or factors responsible for the runting syndrome.

\section{SUMMARY}

The immunological responsiveness of mice was suppressed either by neonatal thymectomy or by adult thymectomy plus whole-body irradiation. The phagocytic activity of the reticuloendothelial system was measured by determining the rate of clearance of 
intravenously injected carbon from the blood. There was no evidence of a hypofunction of the reticuloendothelial system in such animals. Indeed, a slight, although not marked, increase in reticuloendothelial activity was observed in adult thymectomized ir radiated mice and in neonatally thymectomized mice when compared to controls on an age-weight basis. A marked hyperactivity of the reticuloendothelial system was found in mice that developed the runting syndrome following neonatal thymectomy.

\section{ACKNOWLEDGMENTS}

This work was supported in part by the U. S. Atomic Energy Commission and in part by Cancer Research Funds of the University of California.

\section{REFERENCES}

1. Miller, J. F. A. P.; Science 144: 1544, 1964.

2. Schooley, J. C.; Kelly, L. S.; Dobson, E. L.; Finney, C. R.; Havens, V. W., and Cantor, L. N.; Res. J. Reticuloendothelial Soc. 1: 357, 1964.

3. Benacerraf, B.; Halpern, B. N.; Biozzi, G., and Benos, S. A.; Brit. J. Exptl. Pathol. $35: 97,1954$.

4. Parker, H. G., and Finney, C. R.; Am. J. Physiol. 198: 916, 1960.

5. Parrott, D. M. V., and East, J.; Nature 195: 347, 1962.

6. Miller, J. F. A. P.; Proc. Roy, Soc. (London) Ser. B 156: 415, 1962.

7. de Vries, M. J.; van Putten, L. M.; Balner, H., and van Bekkum, D. W.; Rev, Franc. Etudes Clin, et Biol. 9: 381, 1964.

8. Miller, J. F. A. P., and Howard, J. G.; Res. J. Reticuloendothelial Soc. 1: $369,1964$.

9. Miller, J. F. A. P.; Doak, S. M. A., and Cross, A. M.; Proc. Soc. Exptl. Biol. Med. 112: $785,1963$.

10. Cross, A. M.; Leuchars, E., and Miller, J. F. A. P.; J. Expt1. Med. 119: 837, 1964.

11. Parrott, D. M. V., and East, J.; The Thymus in Immunobiology, edited by R. A. Good and A. E. Gabrielsen, New York, Hoeber-Harper, 1964, p. 523.

12. East, J.; Parrott, D. M. V.; Chesterman, F. C., and Pomerance, A.; J. Exptl. Med. 118: $1069,1963$.

13. Howard, J. G.; Brit. J. Expt1. Pathol. 42: 72, 1961.

14. Wilson, R.; Sjodin, K., and Bealmear, M.; Proc. Soc. Expt1. Biol. Med. 117: 237, 1964.

15. McIntire, K. R.; Sell, S., and Miller, J. F. A. P.; Nature 204: 151, 1964.

16. Wilson, R., and Connell, J.; Radiation Protection and Recovery Conference, Houston, Tex., December 4-5, 1964; in Experimental Hematology, No. 7, Biological Division, Oak Ridge National Laboratory, 1964, p. 56.

17. Morrow, S. H., and di Luzio, N. R.; Nature 205: 193, 1965.

Received December, 1965. 


\title{
Heavy Particles and Parkinson's Disease
}

\author{
Robert Tym, John T. Lyman, Robert D. Weyand, Cornelius A. Tobias, \\ Nicholas W. Yanni, James L. Barn and John H. Lawrence
}

A sixty-five-year-old man first noticed a tremor and lack of facility of movement in his right arm and hand in 1959. Both symptoms progressed, and by 1962 the right leg and, to a lesser extent, the left arm and hand were affected. In February 1962, a successful cryothala motomy (left) was performed by Dr. Irving Cooper in New York. The facility of move ment of the right limbs was increased with consequent improvement in gait. The right-sided tremor disappeared also.

The disease process progressed moderately slowly with increase in the symptoms of the left limbs. When first seen in 1963 (and to a greater extent when seen in 1965) his gait and balance were impeded mainly by his left limbs, and there was also some slight return of Parkinsonian rigidity, which impaired facility on the right. There was surprisingly little akinesia.

It was thought that the patient would now benefit from a right thalamotomy, and he expressed a desire to have this performed with heavy-particle irradiation from the 184-inch cyclotron. For localization of the target within the intact skull, three self-tapping screws were inserted into the outer cortex of the skull under local anesthetic. A specially designed stereotaxic frame could thereby be rigidly attached to the head, and the frame could be removed and reapplied onto the skull screws, thus preserving its exact relationship.

The ventricular anatomy was outlined by pneumoencephalography, and the frame coordinates of the target were determined. The region chosen was the inferior ventrolateral nucleus; the target was centered $3.5 \mathrm{~mm}$ above and anterior to the junction of the mid and posterior thirds of the AC-PC line viewed laterally, and $6 \mathrm{~mm}$ medial to the right lateral ventricular line viewed antero-posteriorly on the $\mathrm{X}$ ray.

The 910-MeV helium-ion (alpha-particle) irradiation was performed a few days later when the effects of the pneumoencephalogram had disappeared. For centering the beam of irradiation onto the target, the frame was reapplied to the head and the target coordinates were brought into line with the beam and center of rotation. With the head held in this position, a custom-build mask was applied to the head and face and, in order to eliminate all differential movement of the mask on the head, the mask itself was fixed to the three skull screws. With the head thus held, it was possible to dispense with the stereotactic frame and therefore to avoid unnecessary scattering of the beam. Rotation of the head about the target was controlled by the mask and its rigid bracket. 
The radiation was monitored with a parallel-plate ionization chamber having a collecting electrode $12.7 \mathrm{~mm}$ in diameter; the final beam-collimating aperture had a diameter of $6.35 \mathrm{~mm}$. The radiation was delivered through one plane with rotation. The total rotation was through an arc of 180 degrees; the radiation in all cases entered anteriorly. Measured at the position of the ionization chamber, the total dose delivered was 9,000 rads, the dose rate being approximately $500 \mathrm{rads} / \mathrm{min}$.

The beam profile obtained after the particles had penetrated $6 \mathrm{~g} / \mathrm{cm}^{2}$ of Lucite was no longer rectangular in shape, but more nearly resembled a bell-shaped curve. The relative ionization at the distance of one aperture radius off the beam axis (i. e. the edge of the "ideal" beam) was about 40 to $50 \%$ of the ionization along the beam axis. The various isodose surfaces were well approximated by oblate spheroids. The $90 \%$ isodose surface was very nearly a $4-$ mm diameter sphere. The volume of tissue that received greater than 4,000 rads was estimated to be about $0.16 \mathrm{~cm}^{3}$. At the end of the $30 \mathrm{~min}$ irradiation time the mask and skull screws were removed.

It is now 10 months since this patient was treated, and he appears to be getting along well with some improvement in his gait and possibly some improvement on his left side. Long-term follow-up on this and other patients will be required before the use of heavy particles in treating Parkinson's disease can be evaluated.

Received January, 1966. 


\section{STAFF PUBLICATIONS}

Anger, H. $O$, and Bender, M. A.: Scintillation Cameras, in Nuclear Medicine, edited by W. H. Blahd, New York, McGraw-Hill, 1965, pp. 55-69.

Born, J. L.; Lawrence, J. H.; Linfoot, J. A.; Tobias, C. A.; Manougian, E., and Snyder, N. J.: Hypophyseal Suppression With Heavy Particles in Diabetic Retinopathy, in International Diabetes Federation, Fifth Congress, Toronto, July 21, 1964; On the Nature and Treatment of Diabetes, edited by G. A. Wrensha1l and B. S. Leibel, Amsterdam, Excerpta Medica Foundation, 1965, Chap. 33, pp. 475-482.

Bozzini, C. E.: Decrease in the Number of Erythrogenic Elements in the Blood-Forming Tissues as the Cause of Anemia in Hypophysectomized Rats, Endocrinology 77:977$984,1965$.

D'Angio, G.; Gottschalk, A., and Lawrence, J. H.: Medical Applications of High-Energy Nuclear Particles, in Nuclear Medicine, edited by W. H. Blahd, New York, McGraw-Hill, 1965, pp. 779-798.

Ewing, A. M.; Freeman, N. K., and Lindgren, F. T.: The Analysis of Human Serum Lipoprotein Distributions, in Advances in Lipid Research, Vol. 3, edited by R. Paoletti and D. Kritchevsky, New York and London, Academic Press, 1965, pp. 25-61.

Freifelder, D.: Mechanism of Inactivation of Coliphage T7 by X-Rays, Proc. Natl. Acad. Sci. U. S. 54:128-134, 1965.

Freifelder, D.: A Rapid Technique for the Preparation of Purified Bacteriophage DNA or RNA From Crude Lysates, Biochim. Biophys. Acta 108:318-319, 1965.

Freifelder, D., and Kleinschmidt, A. K.: Single-Strand Breaks in Duplex DNA of Coliphage T7 as Demonstrated by Electron Microscopy, J. Mol. Biol. 14: 271-278, 1965.

Freifelder, D.: Technique for Starvation of Escherichia coli of Thymine, J. Bacteriol. 90: $1153-1154,1965$.

Gottschalk, A., and Anger, H. O.: Use of the Scintillation Camera to Reduce Radioisotope Scanning Time, J. Am. Med. Assoc. 192: 448-452, 1965.

Hildreth, P. E.: Fertilization in Drosophila, II. Time of Inactivation of a Gene Effect, Proc. Natl. Acad. Sci. U. S. 54:736-741, No. 3, 1965.

Lawrence, J. H.: George de Hevesy - a Genius, Letter to the Editor, Intern, J. Appl. Radiation Is otopes 16:511, 1965.

Lawrence, J. H.: The Use of Heavy Particles and the Bragg Peak in Therapy, Acta Isotopica 4: $327-345,1964$.

Lindgren, F. T.; Ewing, A. M.; Freeman, N. K., and Wills, R. D.: A Computer Analysis of Human Lipoprotein Spectra, Ann. N. Y. Acad. Sci. 126:816, 1965.

Rescigno, A. , and Segre, G.: On Some Metric Properties of the Systems of Compartments, Bull. Math. Biophys. 27: 315-323, 1965.

Schaer, L. R., and Anger, H. O.: Organ Visualization Using Scintillation Camera Techniques, Ann. Internal Med. 63: 442-453, 1965.

Shkurkin, C. J., and Lawrence, J. H.: Treatment of Blood Diseases and Malignancy With Radioactive Phosphorous in Relation to Other Therapeutic Agents, in Nuclear Medicine, edited by W. H. Blahd, New York, McGraw-Hill, 1965, pp. 675-699. 
Sondhaus, C. A.: Effect of High-Energy Protons and Alpha Particles on Small Mammals, in Symposium on Protection Against Radiations in Space, Second, Gatlinburg, Tenn., Oct. 12-14, 1964, sponsored by N. A. S. A., U. S. Air Force, U. S. A. E. C. ; Second Symposium on Protection Against Radiations in Space, edited by A. Reetz, Jr., Washington, D. C., National Aeronautics and Space Administration, 1965, pp. 97-103.

Steinkamp, R. C.; Cohen, N. L.; Gaffey, W. R.; McKey, T.; Bron, G. ; Siri, W. E.; Sargent, T. W., and Isaacs, E.: Measures of Body Fat and Related Factors in Normal Adults II. A Simple Clinical Method to Estimate Body Fat and Lean Body Mass, J. Chron. Dis. 18: 1291-1307, 1965.

Steinkamp, R. C.; Cohen, N. L.; Siri, W. E.; Sargent, T. W., and Walsh, H. E. , with the technical assistance of T. McKey, G. Bron, E. M. Hutson, M. H. Rourke, N. D. Kunkel, E. Isaacs, and A. T. Huber: Measures of Body F at and Related Factors in Normal Adults 1. Introduction and Methodology, J. Chron. Dis. 18: 1279-1289, 1965.

Todd, P.: Biological Effects of Heavy Ions, in Symposium on Protection Against Radiations in Space, Second, Gatlinburg, Tennessee, Oct. $12-14,1964$, sponsored by N. A. S. A., U. S. Air Force, U.S.A. E. C.; Second Symposium on Protection Against Radiations in Space, edited by A. Reetz, Jr., National Aeronautics and Space Administration, Washington, D. C., 1965, pp. 105-114.

Van Dyke, D.; Anger, H. O.; Yano, Y., and Bozzini, C.: Bone Blood Flow Shown With $\mathrm{F}^{18}$ and the Positron Camera, Am. J. Physiol. 209:65-70, 1965.

Wallace, R. ; Steward, P. G., and Sondhaus, C.: Primary- and Secondary-Proton Dose Rates in Spheres and Slabs of Tissue, in Symposium on Protection Against Radiations in Space, Second, Gatlinburg, Tennessee, Oct. 12-14, 1964, sponsored by N.A.S. A. , U. S. Air Force, U. S. A. E. C.; Second Symposium on Protection Against Radiations in Space, edited by A. Reetz, Jr., Washington, D. C., National Aeronautics and Space Administration, 1965, pp. 301-329.

Whang, K. S.; Fish, M. B., and Pollycove, M.: Evaluation of Hepatic Photoscanning With Radioactive Colloidal Gold, J. Nucl. Med. 6:494-505, 1965. 